Florida International University FIU Digital Commons

7-20-1993

\title{
Numerical analysis of the pullout problem of a fiber embedded in a matrix : comparison with an approximate analytical solution
}

Ivan Enrique Esparragoza

Florida International University

DOI: $10.25148 /$ etd.FI15101312

Follow this and additional works at: https://digitalcommons.fiu.edu/etd

Part of the Mechanical Engineering Commons

\section{Recommended Citation}

Esparragoza, Ivan Enrique, "Numerical analysis of the pullout problem of a fiber embedded in a matrix : comparison with an approximate analytical solution" (1993). FIU Electronic Theses and Dissertations. 3154.

https://digitalcommons.fiu.edu/etd/3154 


\section{ABSTRACT OF THE THESIS}

Numerical Analysis of the Pullout Problem of a Fiber Embedded in a Matrix: Comparison with an Approximate Analytical Solution

by

Ivan Enrique Esparragoza

Florida International University, 1993

Miami, Florida

Professor Genady P. Cherepanov, Major Professor

The classical problem of pullout of a long elastic rectilinear round bar (fiber) embedded in an elastic half-space (matrix) is considered before and while local debonding occurs. An approximate analytical solution derived from the elasticity theory, the intuitive Saint Venant's principle, the idea of boundary layer in hydrodynamics, and invariant $\Gamma$-integrals is presented. The problem is analyzed numerically by means of the finite element method using the ANSYS program. The cases of loading before and after the initiation of the debonding are studied. Both approaches, analytical and numerical, are compared in order to establish the concidence between them. The discrepancy is very small in the global sense though substantial differences appear at particular points. 


\title{
FLORIDA INTERNATIONAL UNIVERSTTY \\ Miami, Florida
}

Numerical analysis of the pullout problem of a fiber embedded in a matrix: comparison with an approximate analytical solution

\author{
A thesis submitted in partial satisfaction of the \\ requirements for the degree of Master of Sciences \\ in Mechanical Engineering
}

by

Ivan Enrique Esparragoza

1993 

To Professors: Dr. Genady P. Cherepanov

Dr. M. A. Ebadian

Dr. Mohammed El-Sayed

Dr. Cesar Levy

Dr. Kuang-Hsi Wu

This thesis, having been approved in respect to form and mechanical execution, is referred to you for judgement upon its substantial merit.

Dean Gordon Hopkins

College of Engineering and Design

The Thesis of Ivan Enrique Esparragoza is approved.

Dr. Genady P. Cherepanov

Major Professor

Dr. M.A. Ebadian, Chairman

Dr. Mohammed El-Sayed

Dr. Cesar Levy

Date of Examination: July $20^{\text {th }}, 1993$

Dr. Kuang-Hsi Wu

Richard L. Campbell

Division of Graduate Students

Florida International University, 1993 
To my family and to the memory of my father. 


\section{ACKNOWLEDGEMENTS}

It is a privilege for me to have the opportunity to express my gratitude to all those people who, in one or other way, have helped me to complete my master degree and this thesis work.

I would like to thank the members of the thesis committee, Dr. Mohammed ElSayed, Dr. Cesar Levy, and Dr. Kuang-Hsi Wu for their support, advice, and help during the preparation and revision of the original manuscript.

I am deeply grateful with Dr. Genady P. Cherepanov for his invaluable support and guidance throughout the master's program. I would like to thank him too for giving me numerous opportunities for professional development, as well as for providing the topic for this thesis and the central idea for many other research projects. It has been an honor for me to work with Dr. Cherepanov. His influence has awoken in me an interest in investigative work, and I look forward to collaborating with him in future research work.

I would like to express my sincere appreciation to the Department of Mechanical Engineering at FIU, specially to his chairman Dr. M. A. Ebadian for his amiability and availability to solve any problem related with the students.

Finally, I would like to say thanks to my family and all my friends who always believed in me and always encouraged me to reach this goal. 


\section{TABLE OF CONTENTS}

LIST OF FIGURES

vii

LIST OF TABLES

ix

CHAPTER 1. INTRODUCTION $\quad$............................................................ 1

3.1 Introduction $\quad$..................................................................................... 1

3.2 Background $\quad$............................................................................. 2

3.3 Objective ans Significance $\quad$............................................................. 9

CHAPTER 2. APPROXIMATE ANALYTICAL SOLUTION $\quad$.............. 10

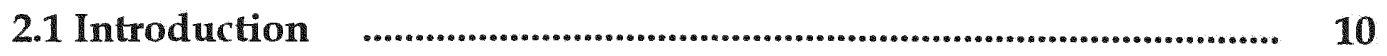

2.2 The Elastic Pullout Problem $\quad$........................................................... 12

2.2.1 Analytical Approach Description $\quad$......................................... 13

2.2.2 Approximate Analytical Solution $\quad$......................................... 18

2.3 The Debonding Problem by Pullout $\quad$................................................ 22

2.3.1 Analytical Approach Description ....................................... 22

2.3.2 Aproximate Analytical Solution $\quad$.......................................... 24

CHAPTER 3. NUMERICAL ANALYSIS $\quad$............................................ 28

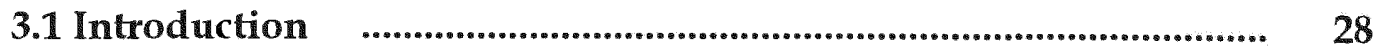

3.2 Modeling Procedure $\quad$.......................................................................... 29

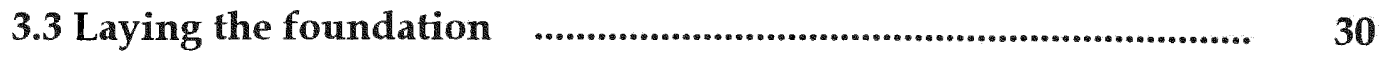


3.2.2 Building the Model $\quad$................................................................... 31

3.2.3 Specifying the Load Data $\quad$.......................................................... 34

3.2.4 Preparing the Solution $\quad$............................................................. 35

3.3 Numerical Solution $\quad$.......................................................................... 36

3.3.1 Results of the Elastic Pullout Problem $\quad$................................. $\quad 37$

3.3.2 Results of the Debonding Problem by Pullout $\quad$................. $\quad 38$

CHAPTER 4. COMPARISON OF NUMERICAL AND ANALYTICAL

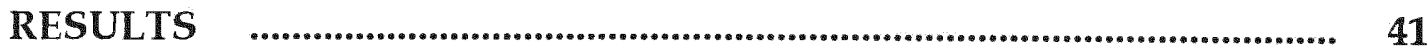

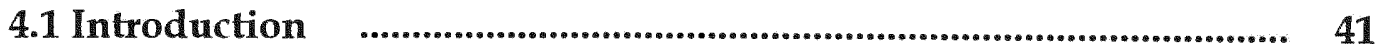

4.2 Comparison of the Elastic Pullout Problem Results $\quad$.................. $\quad 42$

4.2.1 Comparison of Normal Stresses $\quad$.............................................. 43

4.2.2 Comparison of Shear Stresses $\quad$................................................... 45

4.2.3 Comparison of Displacements $\quad$................................................. 46

4.3 Comparison of the Debonding Problem by Pullout $\quad$................... $\quad 47$

4.3.1 Comparison of the Relation between the Load Applied and the Length of Debonding $\quad$........................................................... 48

4.3.2 Comparison of Normal Stresses $\quad$............................................... 50

4.3.3 Comparison of Shear Stresses $\quad$................................................. 51

a. Singularity at the debond zone tip $\quad$.................................... 52

CHAPTER 5. CONCLUSIONS AND PERSPECTIVE $\quad \ldots . . . . . . . . . . . . . . . . . . . . .67$

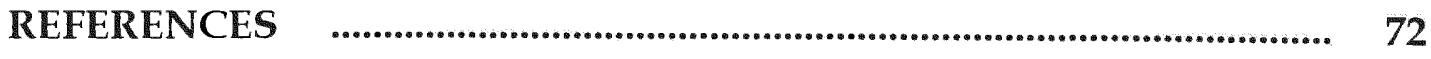

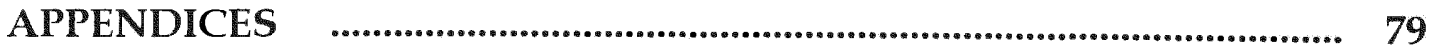




\section{LIST OF FIGURES}

Figure 1. The axisymmetric problem of pullout of a foreign elastic cylindrical bar embedded in a softer elastic half-space ( $m$ is a matrix, and $f$ is a fiber)

Figure 2. The cylindrical domain of non-zero perturbed field. Outside this domain $w$ is zero for $\mathrm{r} \geq \mathrm{r}_{*}$ and $\sigma_{z z}$ is zero for $\mathrm{r}=0$ and $\mathrm{z}>l$. Inside this domain $\sigma_{\mathrm{z}}, \tau_{\mathrm{rz}}$, and $w$ are non-zero; all other stresses and displacements equal to zero

Figure 3. Geometrical design of the numerical procedure for the analysis of the fiber pullout problem using ANSYS version 4.4a

Figure 4. Detail of the local debonding zone. Here,1em and $2 \mathrm{em}$ are the elements corresponding to $l_{D}$ whose Young's modulus was changed in order to model the debonding process in the finite element analysis using the program ANSYS version 4.4a

Figure 5. Graph of $\sigma_{z} / \sigma_{0}$ vs. $z / l$ for $\lambda=0.1$ and $\varepsilon=0.1$

Figure 6. Graph of $\tau_{\mathrm{rz}} / \sigma_{\mathrm{o}}$ vs. $z / l$ for $\lambda=0.1$ and $\varepsilon=0.1$

Figure 7. Graph of $\sigma_{z} / \sigma_{o}$ vs. $z / l$ for $\lambda=0.1$ and $\varepsilon=0.2$

Figure 8. Graph of $\tau_{x z} / \sigma_{o}$ vs. $z / l$ for $\lambda=0.1$ and $\varepsilon=0.2$

Figure 9. Graph of $\sigma_{z} / \sigma_{0}$ vs. $z / l$ for $\lambda=0.01$ and $\varepsilon=0.1$ 56

Figure 10. Graph of $\tau_{\mathrm{rz}} / \sigma_{\mathrm{o}}$ vs. $\mathrm{z} / \mathrm{l}$ for $\lambda=0.01$ and $\varepsilon=0.1$ 56

Figure 11. Graph of $\sigma_{z} / \sigma_{0}$ vs. $z / l$ for $\lambda=0.01$ and $\varepsilon=0.01$

Figure 12. Graph of $\tau_{\mathrm{rz}} / \sigma_{\mathrm{o}}$ vs. $z / l$ for $\lambda=0.01$ and $\varepsilon=0.01$

Figure 13. Graph of $\sigma_{z} / \sigma_{0}$ vs. $z / l$ for $\lambda=0.01$ and $\varepsilon=0.001$ 58

Figure 14. Graph of $\tau_{\mathrm{rz}} / \sigma_{\mathrm{o}}$ vs. $\mathrm{z} / \mathrm{l}$ for $\lambda=0.01$ and $\varepsilon=0.001$ 58

Figure 15. Graph of $\sigma_{o} / \tau_{s}$ vs. $l_{D} / l$ for $\lambda=0.1$ and $\varepsilon=0.1$ 
Figure 16. Graph of $\sigma_{\mathrm{o}} / \tau_{\mathrm{s}}$ vs. $l_{\mathrm{D}} / l$ for $\lambda=0.1$ and $\varepsilon=0.2$

Figure 17. Graph of $\sigma_{\mathrm{o}} / \tau_{\mathrm{s}}$ vs. $\mathrm{l}_{\mathrm{D}} / l$ for $\lambda=0.01$ and $\varepsilon=0.1$

Figure 18. Graph of $\sigma_{o} / \tau_{\mathrm{s}}$ vs. $1_{\mathrm{D}} / l$ for $\lambda=0.01$ and $\varepsilon=0.01$

Figure 19. Graph of $\sigma_{\mathrm{o}} / \tau_{\mathrm{s}}$ vs. $1_{\mathrm{D}} / l$ for $\lambda=0.01$ and $\varepsilon=0.001$

Figure 20. Graph of $\sigma_{z} / \tau_{\mathrm{s}}$ vs. $z / l$ for $\lambda=0.1, \varepsilon=0.1$ and $l_{D} / l=0.1$

Figure 21. Graph of $\tau_{\mathrm{rz}} / \tau_{\mathrm{s}} \mathrm{vs.} z / l$ for $\lambda=0.1, \varepsilon=0.1$ and $l_{D} / l=0.1$

Figure 22. Graph of $\sigma_{z} / \tau_{s}$ vs. $z / l$ for $\lambda=0.1, \varepsilon=0.2$ and $l_{D} / l=0.1$

Figure 23. Graph of $\tau_{\mathrm{rz}} / \tau_{\mathrm{s}}$ vs. $z / l$ for $\lambda=0.1, \varepsilon=0.2$ and $l_{D} / l=0.1$

Figure 24. Graph of $\sigma_{\mathrm{z}} / \tau_{\mathrm{s}}$ vs. $z / l$ for $\lambda=0.01, \varepsilon=0.1$ and $l_{D} / l=0.1$ 64

Figure 25. Graph of $\tau_{\mathrm{rz}} / \tau_{\mathrm{s}} \mathrm{vs} . \mathrm{z} / l$ for $\lambda=0.01, \varepsilon=0.1$ and $l_{D} / l=0.1$ 64

Figure 26. Graph of $\sigma_{\mathrm{z}} / \tau_{\mathrm{s}}$ vs. $\mathrm{z} / l$ for $\lambda=0.01, \varepsilon=0.01$ and $l_{D} / l=0.1$ 65

Figure 27. Graph of $\tau_{\mathrm{rz}} / \tau_{\mathrm{s}} \mathrm{vs.} \mathrm{z} / l$ for $\lambda=0.01, \varepsilon=0.01$ and $l_{D} / l=0.1$ 65

Figure 28. Graph of $\sigma_{\mathrm{z}} / \tau_{\mathrm{s}}$ vs. $\mathrm{z} / \mathrm{l}$ for $\lambda=0.01, \varepsilon=0.001$ and $l_{D} / l=0.1$ 66

Figure 29. Graph of $\tau_{\mathrm{rz}} / \tau_{\mathrm{s}} \mathrm{vs.} z / l$ for $\lambda=0.01, \varepsilon=0.001$ and $l_{D} / l=0.1$ 66 


\section{LIST OF TABLES}

Table 1. Results of the distribution of normalized $\sigma_{z}$ along the total embedded length of the fiber for the elastic pullout problem in the case of $\lambda$ $=0.1$ and $\varepsilon=0.1$

Table 2. Results of the distribution of normalized $\sigma_{z}$ along the total embedded length of the fiber for the elastic pullout problem in the case of $\lambda$ $=0.1$ and $\varepsilon=0.2$

Table 3. Results of the distribution of normalized $\sigma_{z}$ along the total embedded length of the fiber for the elastic pullout problem in the case of $\lambda$ $=0.01$ and $\varepsilon=0.1$

Table 4. Results of the distribution of normalized $\sigma_{\mathrm{z}}$ along the total embedded length of the fiber for the elastic pullout problem in the case of $\lambda$ $=0.01$ and $\varepsilon=0.01$

Table 5. Results of the distribution of normalized $\sigma_{\mathrm{z}}$ along the total embedded length of the fiber for the elastic pullout problem in the case of $\lambda$ $=0.01$ and $\varepsilon=0.001$

Table 6. Results of the distribution of normalized $\tau_{\mathrm{rz}}$ in the interface betwen the fiber and the matrix along the total length of the fiber for the elastic pullout problem in the case $\lambda=0.1$ and $\varepsilon=0.1$

Table 7. Results of the distribution of normalized $\tau_{\mathrm{rz}}$ in the interface betwen the fiber and the matrix along the total length of the fiber for the elastic pullout problem in the case $\lambda=0.1$ and $\varepsilon=0.2$

Table 8. Results of the distribution of normalized $\tau_{\mathrm{rz}}$ in the interface betwen the fiber and the matrix along the total length of the fiber for the elastic pullout problem in the case $\lambda=0.01$ and $\varepsilon=0.1$

Table 9. Results of the distribution of normalized $\tau_{\mathrm{rz}}$ in the interface betwen the fiber and the matrix along the total length of the fiber for the elastic pullout problem in the case $\lambda=0.01$ and $\varepsilon=0.01$ 
Table 10. Results of the distribution of normalized $\tau_{\mathrm{rz}}$ in the interface betwen the fiber and the matrix along the total length of the fiber for the elastic pullout problem in the case $\lambda=0.01$ and $\varepsilon=0.001$

Table 11. Value of the maximum displacement for all the relations $\lambda$ and $\varepsilon$ considered in the numerical analysis of the elastic pullout problem

Table 12. Results of the relation between the normalized load applied and the normalized length of debonding for the case of $\lambda=0.1$ and $\varepsilon=0.1$

Table 13. Results of the relation between the normalized load applied and the normalized length of debonding for the case of $\lambda=0.1$ and $\varepsilon=0.2$

Table 14. Results of the relation between the normalized load applied and the normalized length of debonding for the case of $\lambda=0.01$ and $\varepsilon=0.1 \quad \ldots .$.

Table 15. Results of the relation between the normalized load applied and the normalized length of debonding for the case of $\lambda=0.01$ and $\varepsilon=0.01$

Table 16. Results of the relation between the normalized load applied and the normalized length of debonding for the case of $\lambda=0.01$ and $\varepsilon=0.001 \quad$..

Table 17. Results of the distribution of normalized $\sigma_{z}$ along the total embedded length of the fiber for the debonding problem in the case of $\lambda$ $=0.1, \varepsilon=0.1$ and $l_{D}=1$

Table 18. Results of the distribution of normalized $\sigma_{z}$ along the total embedded length of the fiber for the debonding problem in the case of $\lambda$ $=0.1, \varepsilon=0.2$ and $l_{D}=1$

Table 19. Results of the distribution of normalized $\sigma_{z}$ along the total embedded length of the fiber for the debonding problem in the case of $\lambda$ $=0.01, \varepsilon=0.1$ and $l_{D}=1$

Table 20. Results of the distribution of normalized $\sigma_{z}$ along the total embedded length of the fiber for the debonding problem in the case of $\lambda$ $=0.01, \varepsilon=0.01$ and $l_{D}=1$ 
Table 21. Results of the distribution of normalized $\sigma_{z}$ along the total embedded length of the fiber for the debonding problem in the case of $\lambda$ $=0.01, \varepsilon=0.001$ and $l_{D}=1$

Table 22. Results of the distribution of normalized $\tau_{\mathrm{rz}}$ in the interface between the fiber and matrix along the total length of the fiber for the debonding problem in the case of $\lambda=0.1, \varepsilon=0.1$ and $l_{D}=1$

Table 23. Results of the distribution of normalized $\tau_{\mathrm{rz}}$ in the interface between the fiber and matrix along the total length of the fiber for the debonding problem in the case of $\lambda=0.1, \varepsilon=0.2$ and $l_{D}=1$

Table 24. Results of the distribution of normalized $\tau_{\mathrm{rz}}$ in the interface between the fiber and matrix along the total length of the fiber for the debonding problem in the case of $\lambda=0.01, \varepsilon=0.1$ and $l_{D}=1$

Table 25. Results of the distribution of normalized $\tau_{\mathrm{rz}}$ in the interface between the fiber and matrix along the total length of the fiber for the debonding problem in the case of $\lambda=0.01, \varepsilon=0.01$ and $l_{D}=1$

Table 26. Results of the distribution of normalized $\tau_{\mathrm{rz}}$ in the interface between the fiber and matrix along the total length of the fiber for the debonding problem in the case of $\lambda=0.01, \varepsilon=0.001$ and $l_{D}=1$

Table 27. Comparison of maximum displacement using the dimensionless expression of the bed coefficient for all the relations $\lambda$ and $\varepsilon$ considered in the elastic pullout problem 


\section{CHAPTER 1. INTRODUCTION}

\subsection{Introduction}

Composite materials have become very important in the new technology. They are two or more materials that have been combined to create a new useful material. The main advantage of composites is that they usually exhibit the best qualities of their constituents and often some qualities that neither constituents possesses. Composites are formed in order to improve some properties such as strength, stiffness, fatigue life, conductivity and others.

Because of the inherent nature of composites materials, they are usually studied from the macro and micro mechanics behavior. Macro mechanics behavior is the study of composite materials wherein the material is presumed homogeneous and the effects of the constituent materials are detected only as averaged apparent properties of the composite. Micro mechanics is the study of the interactions of the constituent materials.

Unfortunately, many difficulties arising in the design or employment of composite materials are caused by the complexity and poor understanding of the interactions between the composite components. According to Cherepanov (1993) in the treatment of some novel approaches in mechanics of composites, so far, there are no generally recognized theories of such phenomena as: fiber reinforcement, adhesion/cohesion of two materials, decohesion / debonding / delamination of fibers, and so on. Due to this fact, many researchers have been involved in investigations in those areas. In this work, attention will be focused 
on one of the most famous problem in mechanics of composite which is the pullout of a fiber embedded in a matrix.

In the context of solid mechanics, this type of investigation is conducive to a better understanding of the behavior of fiber-reinforced composites, to which the load transfer characteristics between the matrix and the reinforcement is of importance. Of interest in this work is the response of the system under the action of axial loading such as that incurred by axial force at the top of the fiber.

\subsection{Background}

As was explained above, the interactions between fibers and matrix are complex, and imperfectly understood. Cox (1952) was probably the first who attempted to explain the reinforcing effect, and his study was based entirely on elastic interactions. This idea is now called the shear lag theory, and it was improved by Kelly and Davies (1965); however, it basically explains only the behavior of composites at low stresses.

The fundamental role of pullout problem in reinforcement and toughness of composite materials was originally understood probably by Kelly and Davies (1965). Muki and Sternberg (1969) were the first to study the problem analytically. In their treatment of on an axially-loaded infinite rod embedded in a medium of infinite extent, the influence of the deformability of the embedment on its mechanical interaction with the surrounding medium is illustrated. Of greater importance, though, is their subsequent contribution to the more difficult problem of axial load diffusion from a partially embedded rod in a semi-infinite 
medium (Muki and Sternberg, 1970). In that treatment it is shown that it is possible to obtain a consistent formulation for the structure-medium interaction problem on the occasion that the engineering approach of treating the rod as a one-dimensional elastic continuum is adopted. The model by Muki and Sternberg was used to study such problems as load-transfer to a half-space from a partially embedded axially loaded rod and load-absorption by a semi-infinite fiber in a remotely stressed, fully-infinite matrix (Sternberg, 1970). Muki and Sternberg's model replaces the fiber-matrix system of the problem with an extended matrix occupying the volume originally containing both the fiber and the matrix and possessing the same elastic properties as the original matrix. This extended matrix is in turn reinforced by a "fictitious stiffener" whose modulus of elasticity when taken in sum with that of the extended matrix is equal to that of the original fiber. This stiffener is taken to be a one-dimensional elastic continuum bonded to the extended matrix in such a way that the axial strain in the stiffener is equal to the average extensional strain of the extended matrix in the volume occupied by and in the direction of the original fiber. Poisson's effect in the stiffener, and therefore in the fiber, is not taken into account. Finally, "bond forces" are regarded as body forces uniformly distributed over disks perpendicular to the axis of the fiber and load carried by the original fiber is equated with the sum of the stiffener load and the resultant load carried by the extended matrix in the bonded region.

The model of Muki and Sternberg was modified by Pak (1989). Pak's work is concerned with the analysis of the response of a partially embedded bar under lateral loading. In this work, the loading is assumed to be applied at the unembedded end of the bar and may, in general, be a combination of horizontal 
shear forces and moments. The concept of a "fictitious stiffener" replacing the original fiber and treated as a one-dimensional elastic continuum was again employed. In this case, however, lateral displacement of the stiffener was taken to equal lateral displacement in the extended matrix along the centroidal axis of the original fiber and Bernoulli-Euler bending beam theory was used to describe the behavior of the stiffener. Body-force field distributions corresponding to laterally-loaded rigid disks embedded in the matrix along the axis of the fiber were adopted as the "bond forces"

Another model was recently advanced by Slaughter and Sanders (1991). In this proposed model, the effect of the fiber on the matrix is assumed to be approximated by unknown distributions of axial forces and dilatations in an elastic space along the line where the fiber axis would lie. Mathematically the elastic field in the matrix is represented in terms of integrals with kernel functions corresponding to concentrated loads and dilatations. The fiber is modeled by a one-dimensional rod theory in which Poisson expansions and contractions are allowed. The two unknown distributions are determined by enforcing fiber equilibrium and continuity of tractions and displacements at the fiber-matrix interface leading mathematically to a pair of coupled integral equations. This model would seem to be conceptually "clean"; however, there is a difficulty. A concentrated axial force applied to the (model) fiber necessarily produces a discontinuity in axial strain. On the other hand, any distribution whatever of axial forces and dilatations according to the model produces continuous axial strains in the matrix at the fiber-matrix interface. This fundamental inconsistency is avoided by introducing an approximate expression for the axial strain in the matrix which has the proper discontinuity but which 
differs from the exact expression over a distance the order of a fiber radius. It is difficult to give a rigorous assessment of the errors involved in the approximate theory but it is thought to be accurate except within distances the order of a fiber radius from concentrated loads or other discontinuities. In contrast to the model used by Muki and Sternberg, this approach treats the load between the fiber and matrix in a manner which explicitly includes tangential tractions across the interface and therefore affords one more flexibility in examining systems where interface conditions are an issue. Furthermore, the fundamental elastostatic solutions in application in this model are those for a point force and a point dilatation. These solutions are much less cumbersome than the disk of uniform loading (or laterally-loaded rigid disk) required in Muki and Sternberg's model. It is important to notice that the work by Slaughter and Sanders (1991) considers only the case of $\lambda=0.2, \varepsilon=0.5, v_{\mathrm{m}}=1 / 4$ and $v_{\mathrm{f}}=1 / 3$, and this model is compared with that studied by Muki and Sternberg (1969). Such a kind of model has no considerable practical importance for composite materials because, for practical purposes, $\lambda$ varies from $10^{-5}$ to $10^{-3}$ and $\varepsilon$ varies from $10^{-3}$ to $5.10^{-2}$.

The classical pullout problem has been studied considering its two clearly defined stages: before and while local debonding occurs. For this case, the elastic pullout problem is a necessary prelude to fiber debonding, see, e.g. Lawrence (1972), Takaku and Arridge (1973), Phillips (1974), Wells and Beaumont (1985), Budiansky, Hutchinson and Evans (1986), Delale (1988), and Becher et al. (1988), Kerans and Parthasarathy (1991), Cherepanov and Esparragoza (1992). Besides, a tremendous list of papers on interfacial debonding can be found in Cherepanov (1983), Friedrich (1989), Rice, Suo and Wang (1990), Bao and Hui (1990), Hseuh 
(1990), Hutchinson and Jensen (1990), Evans (1991), Hutchinson and Suo (1992), Cherepanov and Esparragoza (1993).

The importance of the brittle fiber/matrix interface on the mechanical behavior of ceramic composites has led to several recent studies measuring the force necessary to slip a fiber by pushing out its end with an indenter (Marshall and Oliver, (1987), Mandell et al. (1987), Brun and Singh (1988) and Morscher et al. (1990)) and with a flat ended probe (Bright et al. (1989)). Most of the analyses assumed the fiber/matrix interface to be fully debonded so that the only resistance to slippage was friction. Rigorous analytical solutions to the fiber slippage problem carried out by Dollar and Steif (1988) are restricted to cases in which the fiber and matrix have the same elastic properties and the initial residual stress presented in the composite is a constant clamping pressure. An analytical model for the transfer of stress between fiber and matrix at an interface where there is either perfect bonding or where friction is governed by Coulomb's law was given by McCartney (1989). A simplified model for fiber pushout tests based on a shear-lag theory was proposed by Hseuh (1990).

Recently, the problem of fiber/matrix interface debonding has received much attention. There have been two approaches to the debonding problem. One is based on a maximum shear-stress criterion, as carried out by Hsueh (1990) and Li et al. (1991), and the other based on a fracture mechanics (mode II crack growth) approach, as conducted by Gao et al. (1988), Hutchinson and Jensen (1990) and Kerans and Parthasarathy (1991). When the fiber is partially debonded, clamping stresses due to mismatch in the thermal expansion coefficients of the fiber and the matrix act on the fiber, giving rise to frictional 
shear stresses at the fiber/matrix interface. Gao et al. (1988) analyzed the problem, including the effects of friction, using a model based on shear-lag theory. However, they ignored the effects of axial or shear residual stresses in the specimens. Effects of the axial and radial residual stresses on the fiber pullout stresses were considered by Hsueh (1990). Hsueh (1990) also analyzed debonding due to residual thermal stresses using the interfacial bond strength as the criterion for debonding. Hutchinson and Jensen (1990) used a fracture mechanics approach to give a comprehensive treatment of the fiber pullout problem for a semi-infinite composite including all residual stresses due to thermal cool-down. The effects of axial residual stresses on fiber pullout and pushout problems were taken into account by Kerans and Parthasarathy (1991), who also included the effects of fiber surface roughness in their model.

In all the analyses of fiber pushout or pullout tests in the past, the following assumptions were made: a) In most cases the composite was assumed to be either fully undebonded or fully debonded at the beginning of the test. The possibility of a finite initial debonded zone was considered by Hutchinson and Jensen (1990) and Hsueh (1990) for the fiber pullout case. b) In most analyses of the fiber pushout or pullout tests, residual stress was taken as a constant clamping stress, although Hsueh (1990) and Kerans and Parthasarathy (1991) also included a constant axial residual stress term. A full residual stress field due to thermal cool down was included by Hutchinson and Jensen (1990) in their analysis of the fiber pullout test of a semi-infinite composite. A similar accounting of the residual stress field was also given by McCartney (1989) for the case of a fully debonded frictional interface. c) In all cases, the composite was assumed to be semi-infinite. 
At this point, it is important to cite other works that have contributed to the analysis of the pullout problem. Many new and useful suggestions taking into account other facts such as residual stresses, non-constant interfacial bond strength, imperfect bonding and temperature effects were studied in Brun and Singh (1988), Mura, Brittain and Faber (1990), Chen and Hui (1990), Kishi, Ewoki and Tsuda (1992), and Kim, Baillie and Mai (1991).

Some ideas originated in the study of the interactions between fiber and matrix such as the shear-lag theory and the method for analyzing fiber load-diffusion problems developed by Slaughter and Sanders (1991) have been used for the analysis of crack-bridging problems in composites. It has been demonstrated how a steady-state crack, growing normal to an aligned array of reinforcing fibers, can be modeled by analyzing a configuration in which there are no fibers but a continuous distribution of springs restraining the two crack faces (Budiansky and Amazigo, 1989). Thus, they used a shear-lag model to determine an equivalent spring stiffness for their model. Slaughter (1992) proposed a self-consistent model for determining the equivalent spring constant in fiber crack-bridging problem using the method for analyzing fiber loaddiffusion problem. Similarly, the fracture mechanics approach has been applied to the pullout problem. See Gao et al. (1988), Hutchinson and Jensen (1990), Stang, Li and Shah (1990), and Kerans and Parthasarathy (1991).

Many important issues of pullout problems such as friction effect, interfacial micro-cracking and interface cracking strength were studied in Bright, Shetty, Griffin and Limays (1989), Delale and Xu (1991), Nair (1990), Ortiz and Blume (1990), Parthasarathy, Jero and Kerans (1991), Sutcu and Hilling (1990), Thouless 
and Evans (1988), Thouless, Sbaizero, Sigl and Evans (1989), and Zimmerman, Langford and Dickinson (1991).

\subsection{Objective and Significance}

The objective of this thesis is to determine, by means of the finite element method, the distribution of the normal stress in the cross section of the fiber and the shear stress in the interface between the fiber and the matrix along the total length of the fiber during the pullout process of a fiber embedded in a matrix before and while local debonding occurs. Also it is important to determine the maximum load that can be applied in order to start debonding and, mainly, to determine the maximum load that can be applied until which the length of debonding grows stable and, consequently, the fiber composite can continue working.

The significance of this work is that this analysis allows us to have new tools for the study of the pullout problem of a fiber embedded in a matrix, taking into account initial or residual, inelastic behavior of the fiber and/or the matrix, interactions of many fibers, temperature effects and so on. In addition, because there is no experimental data available, this work serves to establish the coincidence between the numerical solution and a new approximate analytical solution of this problem presented in Chapter 2 which is simpler than those developed by other authors. 


\section{CHAPTER 2. APPROXIMATE ANALYTICAL SOLUTION}

\subsection{Introduction}

In this Chapter the approximate analytical solution is presented. For this purpose a linearly elastic round cylinder embedded in a linearly elastic halfspace of another material on a finite length is considered (Fig. 1). The force $F$ is applied to the open end of the cylinder. The boundary of the half-space and lateral open surface of the cylinder are considered to be free of traction. Residual or initial stresses of technological origin are ignored in this work.

The following designations are accepted: $r_{0^{\prime}}$ the radius of the cylinder; $l$, the length of the embedded part of the cylinder; $E_{f}$ and $E_{m^{\prime}}$ the Young's modulus of the fiber (cylinder) and matrix (the bulk material) respectively; $v_{\mathrm{f}}$ and $v_{\mathrm{m}^{\prime}}$ the Poisson's ratio of the fiber and matrix respectively.

The following dimensionless quantities are also introduced:

$$
\lambda=\frac{r_{o}}{l} \quad \text { and } \quad \varepsilon=\frac{E_{m}}{E_{f}} \text {. }
$$

According to the Saint Venant principle the length of the open cylinder part, not embedded into a matrix, as well as the distribution of $\mathrm{F}$ along a top cross-section of the cylinder are not essential, if the length of the open part is much greater than the radius of the cylinder, which is assumed in the following text. 


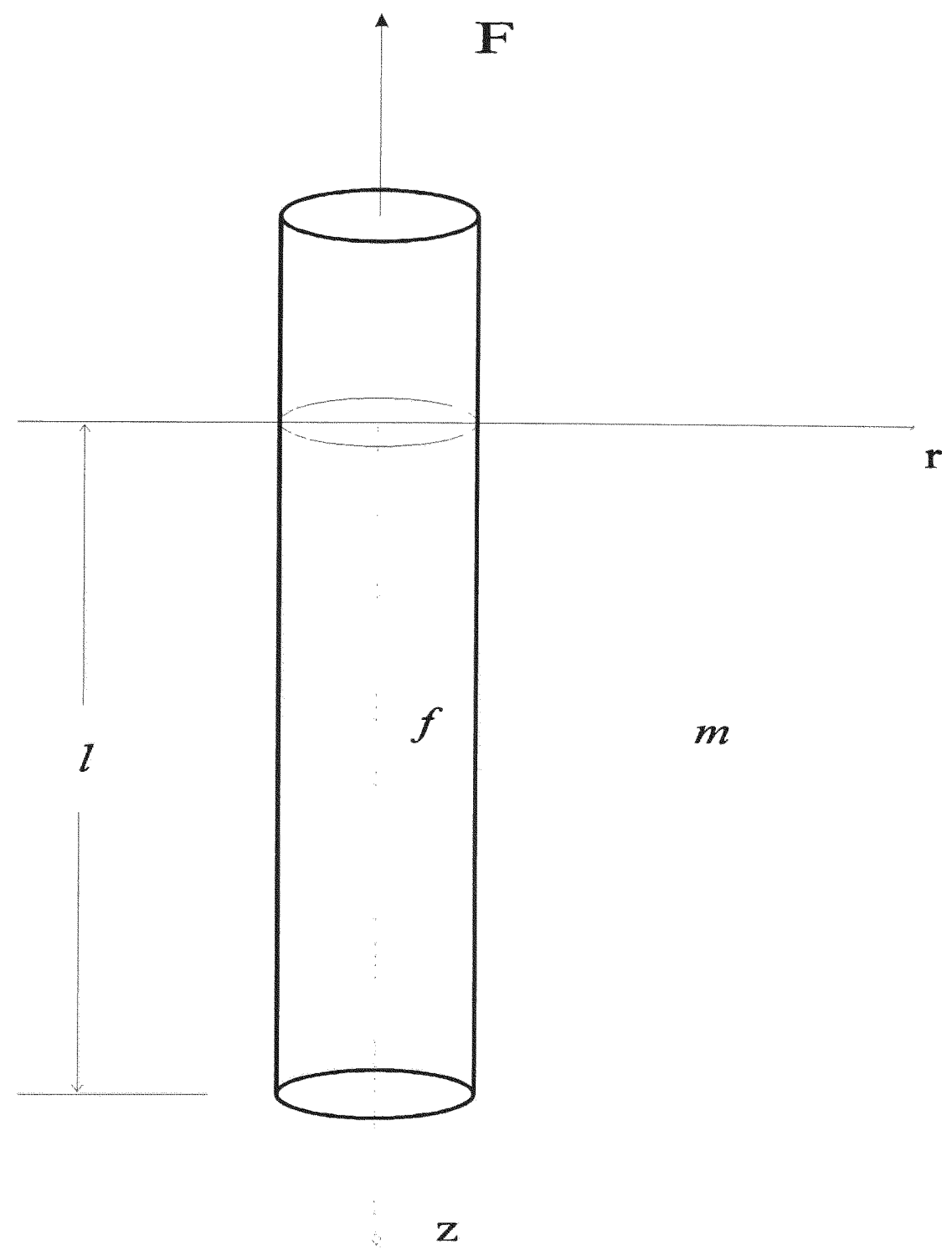

Figure 1. The axisymmetric problem of pullout of a foreign elastic cylindrical bar embedded in a softer elastic half-space ( $m$ is a matrix, and $f$ is a fiber). 
The problem under consideration is close to the classical problem of a thin high modulus fiber in a homogeneous elastic space subjected to a longitudinal extension, or to the problem of a thin conductive cylinder in a homogeneous dielectric matter under the longitudinal electrostatic field. Unsuccessful attempts to solve this problem were undertaken in Landau and Lifshitz (1960), Cherepanov (1981) and Eshelby (1982). Eshelby quoted also the earlier work by Hallen, Sommerfield, Lorentz, Taylor, and Van-Dyke who tried to find an efficient analytical solution to the problem. Such a solution was provided in Cherepanov (1983) using a special approach including a fitting index determined by a computer simulation. In Nikishkov and Cherepanov (1984), numerical experiments on the solution of this problem were treated. The last approach is applied in this Chapter for an efficient analytical solution to the problem of pullout under study.

The theoretical approaches suggested by other authors for solution of pullout problem are distinct from the approach utilized here. As a rule they do not take a full advantage of small $\varepsilon$ and $\lambda$ in order to combine a sufficient accuracy and a necessary conciseness of general approach needed for the more difficult applications.

\subsection{The Elastic Pullout Problem}

For this case, the following condition is assumed: there exists a perfect bonding between the fiber and the matrix, which means that the condition of the no-slip and no-opening bonding holds over all the interface including the lateral surface and the bottom . 


\subsubsection{Analytical Approach Description}

The problem is axisymmetric and the cylindrical coordinates $\mathrm{r}$ and $\mathrm{z}$ are convenient to use, so that $\mathrm{z}$ is the axis of symmetry and $\mathrm{r}$ is the radial distance from the axis. The foreign cylinder occupies the domain $\mathbf{r}<r_{y^{\prime}}$ and $z<l$, and the free boundary of the half-space corresponds to $z=0$ on Fig. 1. Across the interface all the displacements and tractions are continuous.

The case when both $\lambda$ and $\varepsilon$ are small is under study:

$$
\lambda<<\quad \varepsilon<<1
$$

It means that the length and the elastic modulus of the cylinder (fiber) are considered to be much greater in comparison with the radius of the fiber and the elastic modulus of the bulk material (matrix), respectively. This is the case of most practical relevancy to composite materials.

In this case, the approximate analytical approach brought in Cherepanov (1983) is used. According to this approach the displacement and stress-strain field in the fiber is described by the following three functions:

$$
\sigma=\sigma(z), \quad \tau=\tau(z) \quad \text { and } \quad W=W(z)
$$

Here $\sigma$ is the mean normal stress, $\sigma_{\mathrm{zz}}$ in a cross-section of the fiber; $\tau$ is the mean shear stress, $\tau_{\mathrm{rz}}$ on the lateral surface of the fiber; and $W$ is the mean displacement of a cross-section of the fiber along the z-axis. 
The following two equations hold according to this approach:

Equilibrium equation

$$
2 \tau(z)+r_{o} \sigma(z)=0
$$

Hooke's law

$$
\sigma=E_{f} W^{\prime}(z)
$$

(') denotes the derivative with respect to $\mathrm{z}$.

According to the same approach the perturbed field of stresses, strains and displacements in matrix exists only inside the cylindrical domain $r<r_{*}, 0<z<l$ shows in Fig. 2, where $r_{*}$ should be found from the following equation of fitting according to Cherepanov (1983):

$$
\frac{r_{*}}{r_{o}}=\frac{l^{\alpha}}{r_{o}^{\alpha}}
$$

Here $\alpha$ is the unknown fitting index subject to be determined. Inside this cylindrical domain the fields of strain, stress and displacement are described by the following three functions:

$$
\sigma_{z}=\sigma_{z}(r, z), \quad \tau_{r z}=\tau_{r z}(r, z), \quad w=w(r, z)
$$

Here $w$ is the $\mathrm{z}$ component of the displacement vector; and $\sigma_{\mathrm{z}}$ and $\tau_{\mathrm{rz}}$ are respective components of the stress tensor. All other components of the stress tensor and displacement vector are considered small enough to be ignored. 
Inside the cylindrical domain $r<r_{*}, 0<z<l$ the following three equations are assumed to be valid:

Equilibrium equation

$$
\frac{\partial}{\partial r} r \tau_{r z}=0
$$

Hooke's law

$$
\begin{aligned}
& \sigma_{z}=E_{m} \frac{\partial w}{\partial z} \quad \tau_{r z}=G_{m} \frac{\partial w}{\partial r} \\
& 2 G_{m}=\frac{E_{m}}{1+v_{m}}
\end{aligned}
$$

Outside this domain, the displacement $w$ is postulated small enough to be ignored for $\mathbf{r} \geq \mathbf{r}_{*}$, and the stress $\sigma_{z z}$ is accepted small enough to be ignored for $\mathbf{r}<r_{0}$ and $\mathrm{z}>1$.

In view of the latter conditions, the following boundary conditions should be met by the sought solution (Fig. 2):

The conditions of equilibrium and bonding at the interface are:

$$
\tau=\tau_{r s}, \quad w=W \quad \text { when } r=r_{o}
$$

The condition of displacement continuity at $r=r_{*}$ is:

$$
w=0 \quad \text { when } r=r_{*}
$$

The condition of equilibrium at $z=l$ in the fiber is: 


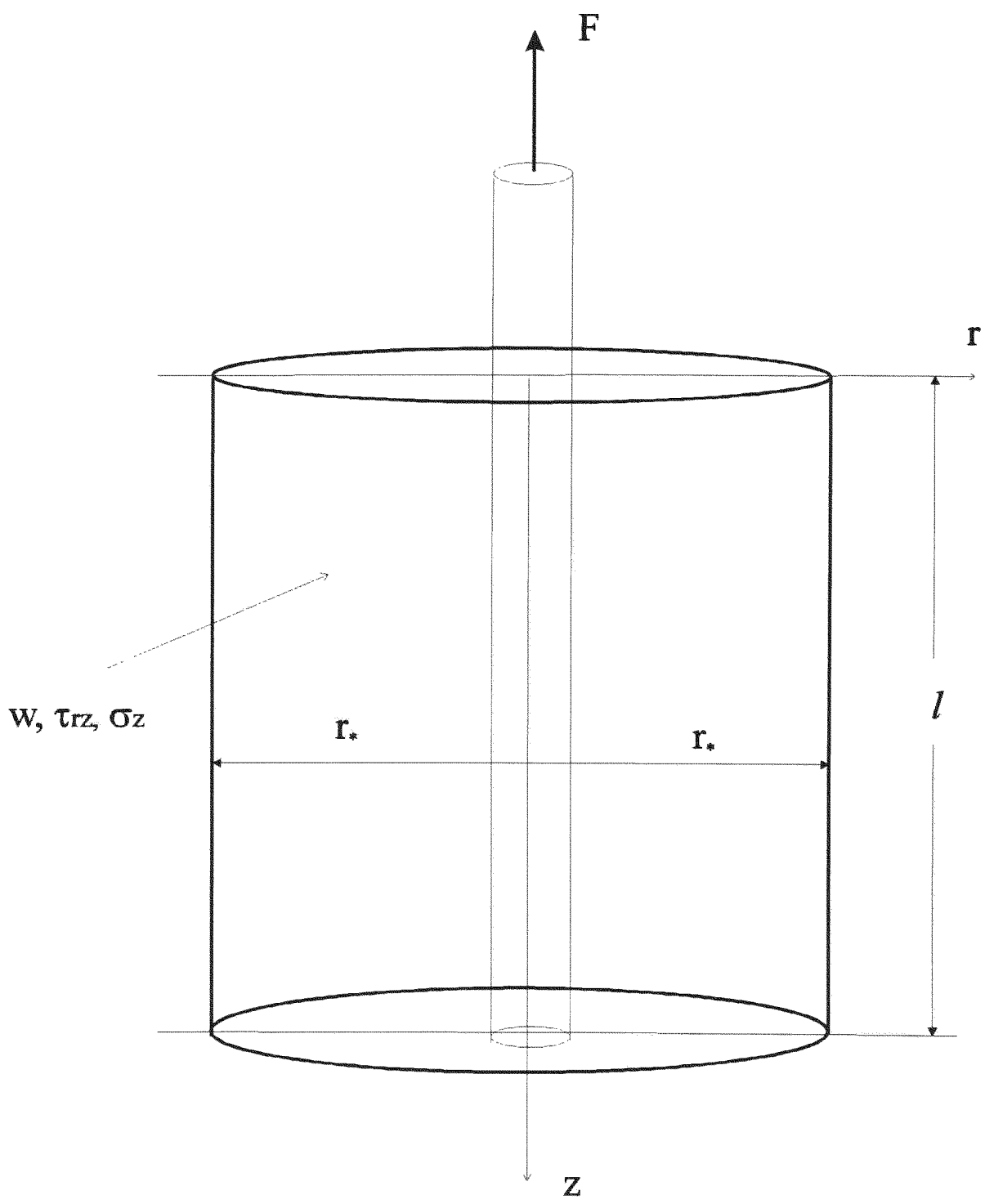

Figure 2. The cylindrical domain of non-zero perturbed field. Outside this domain $w$ is zero for $r \geq r_{*}$ and $\sigma_{z z}$ is zero for $r=0$ and $z>l$. Inside this domain $\sigma_{z^{\prime}} \tau_{\mathrm{rz}}$ and $\mathrm{w}$ are non-zero; all other stresses and displacements equal to zero. 


$$
\sigma=0 \quad \text { when } z=l
$$

The condition of equilibrium at $z=0$ in the fiber is:

$$
\sigma=\sigma_{o} \quad \sigma_{o}=\frac{F}{\pi r_{o}^{2}}
$$

The system of Eqs. (2.3) to (2.13) totally describes the approach undertaken to find the efficient analytical solution to pullout problem under study. One additional condition necessary to define fitting index $\alpha$ will be elucidated below. (It appears that the value of $\alpha$ very slightly depends on the choice of the condition).

Some arguments supporting this approach are brought in Cherepanov (1983). They are based on the ideas of the intuitive Saint Venant's theory of beams, on the ideas of boundary layer in hydrodynamics and on invariant $\Gamma$-integrals. However, it should be noted that $\mathbf{r}_{*}$ is not small in comparison to $l$, so that the perturbed domain on Fig. 2 does not look like a very thin boundary layer of a viscous fluid near stream lined bodies. Analogously to the classical theories of beams, plates or shells, which are factually intuitive, the present approach is substantiated, first and foremost, by excellent agreement of its analytical results with those of numerical experiments. 


\subsubsection{Approximate Analytical Solution}

Substituting $\tau_{\mathrm{rz}}$ of Eq. (2.9) in Eq. (2.8), solving the obtained equation with respect to $w$ and satisfying the boundary conditions in Eq. (2.10), the following set of equations is found:

$$
\begin{aligned}
& w=\frac{r_{o}}{G_{m}} \tau(z) \ln \frac{r}{r_{o}}+W(z) \\
& \tau_{r z}=\tau(z) \frac{r_{o}}{r} \\
& \sigma_{z}=E_{m} W^{\prime}(z)+2\left(1+v_{m}\right) r_{o} \tau(z) \ln \frac{r}{r_{o}}
\end{aligned}
$$

The last two equations were found from Eqs. (2.9) and (2.14). From Eqs. (2.11) and (2.14), it follows:

$$
G_{m} W(z)+r_{o} \tau(z) \ln \frac{r}{r_{o}}=0
$$

This equation, which is basic in the problem under study according to the approach of Section 2.2.1, can be also derived using invariant $\Gamma$-integral, similarly to Cherepanov (1983).

The system of Eqs. (2.4), (2.5) and (2.17) is closed with respect to $\tau(\mathrm{z}), \sigma(\mathrm{z})$ and $W(\mathrm{z})$. Its general solution is found by means of the method of elimination. The following results are obtained using hyperbolic functions: 


$$
\begin{aligned}
& W=C_{1} \sinh \frac{k z}{l}+C_{2} \cosh \frac{k z}{l} \\
& \sigma=\frac{k E_{f}}{l}\left(C_{1} \cosh \frac{k z}{l}+C_{2} \sinh \frac{k z}{l}\right) \\
& \tau=-\frac{G_{m} W}{r_{o} \ln \frac{r_{*}}{r_{o}}}
\end{aligned}
$$

Here:

$$
k=\frac{\sqrt{\varepsilon}}{\lambda \sqrt{\left(1+v_{m}\right) \ln \frac{r_{*}}{r_{o}}}}
$$

$\mathrm{C}_{1}$ and $\mathrm{C}_{2}$ are arbitrary constants.

Using Eqs. (2.12) and (2.13) in Eq. (2.19) $C_{1}$ and $C_{2}$ are found:

$$
C_{1}=\frac{\sigma_{o} l}{k E_{f}} \quad C_{2}=-\frac{\sigma_{0} l}{k E_{f}} \frac{1}{\tanh (k)}
$$

Substituting Eq. (2.22) into Eqs. (2.18) to (2.20) the final solution to the problem is found:

$$
\begin{aligned}
& W=-\frac{\sigma_{o} l}{k E_{f} \sinh (k)} \cosh \left(k\left(1-\frac{z}{l}\right)\right) \\
& \sigma=\frac{\sigma_{o}}{\sinh (k)} \sinh \left(k\left(1-\frac{z}{l}\right)\right)
\end{aligned}
$$




$$
\tau=\frac{\lambda k \sigma_{o}}{2 \sinh (k)} \cosh \left(k\left(1-\frac{z}{l}\right)\right)
$$

It follows that the maximum stresses in the fiber are equal to:

$$
\sigma_{\max }=\sigma_{o} \quad \text { and } \quad \tau_{\max }=\frac{\lambda k \sigma_{o}}{2} \frac{1}{\tanh (k)} \quad \text { at } \mathrm{z}=0
$$

In the limiting cases of small and large $\mathrm{k}$ it can be proved:

$$
\begin{array}{ll}
\text { for } \mathrm{k}>>1 & \tau_{\max }=\frac{\lambda k \sigma_{o}}{2} \\
\text { for } \mathrm{k}<<1 & \tau_{\max }=\frac{\lambda \sigma_{o}}{2}
\end{array}
$$

According to Eq. (2.23) the displacement $W$ at the ends of the fiber equals:

$$
\begin{aligned}
& \text { for } z=0 \quad W_{\max }=-\frac{\sigma_{o} l \cosh (k)}{k E_{f} \sinh (k)} \\
& \text { for } z=l \quad W_{\min }=-\frac{\sigma_{o} l}{k E_{f} \sinh (k)}
\end{aligned}
$$

From Eq. (2.28) it follows that the elastic bed coefficient, $B$, of the forcedisplacement, or $\mathrm{F}-W_{\max }$, diagram

$$
F=B W_{\max }
$$

where 


$$
B=\pi \lambda k r_{o} E_{f} \tanh (k)
$$

This formula is reliable, at least, for $\mathrm{k}>>1$ when $W_{\min }<<W_{\max }$.

The fitting index $\alpha$ defined by Eq. (2.6) can be found from a numerical or physical experiment using a requirement of coincidence or best fitting of a characteristic quantity of convenience in the problem. For example, it can be required that for certain values of $\varepsilon, \lambda, k$ and $v_{m}$ the value $\tau_{\max } / \sigma_{o}$ determined by Eq. (2.27) would coincide with experimental value. As shown below in Chapter 4 the results of numerical experiments confirm very well the approximate analytical solution Eqs. (2.23) to (2.25) if the value of the fitting index is taken from Cherepanov (1983)

$$
\alpha=0.738
$$

The value of $\alpha$ very slightly depends on the choice of fitting condition.

Substituting Eq. (2.31) into Eq. (2.6) and then Eq. (2.6) into Eq. (2.21) k can be defined as:

$$
k^{2}=\frac{1.355 \varepsilon}{\lambda^{2}\left(1+v_{m}\right) \ln \left(\frac{1}{\lambda}\right)}
$$




\subsection{The Debonding Problem by Pullout}

Here, a new designation is introduced: $l_{D}$, the length of debonding measured from $z=0$.

Consequently, the following dimensionless quantity is also introduced:

$$
\lambda_{D}=\frac{r_{o}}{l-l_{D}}
$$

The following conditions will be assumed: (a) a part of the interface adjacent to the free boundary of the half-space is debonded, so that, the condition of zero tractions is assumed to hold over the former interface; and (b) the remaining part of the interface is bonded, hence, the condition of the no-slip and no-opening is assumed to hold over the latter interface including the bottom.

\subsubsection{Analytical Approach Description}

For this case the same analytical approach used in Section 2.2.1 is valid; therefore, Eqs. (2.2) to (2.5) and Eqs. (2.7) to (2.9) will be used. It should be noticed that for this case Eqs. (2.8) and (2.9) are valid inside the cylindrical domain $\mathbf{r}<r_{*}$ and $l_{D}<\mathrm{z}<l$.

Nevertheless, Eq. (2.6) should be rewritten taking into account the length of debonding in the following form: 


$$
\frac{r_{*}}{r_{o}}=\frac{\left(l-l_{D}\right)^{\alpha}}{r_{o}^{\alpha}}
$$

Now, the following boundary conditions should be met by the sought solution:

The conditions of equilibrium and bonding at the interface are:

$$
\tau=\tau_{r z}, \quad w=W \quad \text { when } \quad r=r_{o}
$$

The condition of displacement continuity at $\mathrm{r}=\boldsymbol{r}_{*}$ is:

$$
w=0 \quad \text { when } \quad r=r_{*}
$$

The condition at $z=l$ in the fiber is:

$$
\sigma=0 \quad \text { when } \quad \mathrm{z}=l
$$

At the beginning of local debonding zone, the shear stress is maximum and the normal stress is given, as follows:

$$
\tau=\tau_{s} \text { and } \quad \sigma=\sigma_{o} \quad\left(\sigma_{o}=\frac{F}{\pi r_{o}^{2}}\right) \quad \text { when } \quad \mathrm{z}=l_{D}
$$

where $\tau_{\mathrm{S}}$ is the limiting shear stress at the interface, which characterizes the ultimate strength of bonding. 
The system of Eqs. (2.3) to (2.5), (2.7) to (2.9), and (2.34) to (2.38) totally describes the approach undertaken to find the efficient analytical solution to pullout problem considering debonding. This approach is based on the same ideas and principles explained in Section 2.2.1.

\subsubsection{Approximate Analytical Solution}

Using the same procedure applied in Section 2.2.2 combining Eqs. (2.9), (2.7) and (2.35), the same set of Eqs. (2.14) to (2.16) is found.

From Eqs. (2.14) and (2.36) it follows:

$$
G_{m} W(z)+r_{o} \tau(z) \ln \frac{r}{r_{o}}=0
$$

Eq. (2.39) is the basic equation in the problem under study according to the approach explained in Section 2.2.1.

The system of Eqs. (2.4), (2.5) and (2.39) is closed with respect to $\tau(z), \sigma(z)$ and $W(\mathrm{z})$. Its general solution is found by means of the method of elimination. The following results are obtained using hyperbolic functions and taking into account the length of debonding:

$$
W=C_{1} \sinh \frac{k_{D} z}{l-l_{D}}+C_{2} \cosh \frac{k_{D} z}{l-l_{D}}
$$




$$
\begin{aligned}
& \sigma=\frac{k_{D} E_{f}}{l-l_{D}}\left(C_{1} \cosh \frac{k_{D} z}{l-l_{D}}+C_{2} \sinh \frac{k_{D} z}{l-l_{D}}\right) \\
& \tau=-\frac{G_{m} W}{r_{o} \ln \frac{r_{*}}{r_{o}}}
\end{aligned}
$$

Here:

$$
k_{D}=\frac{\sqrt{\varepsilon}}{\lambda_{D} \sqrt{\left(1+v_{m}\right) \ln \frac{r_{*}}{r_{o}}}}
$$

$\mathrm{C}_{1}$ and $\mathrm{C}_{2}$ are arbitrary constants.

Using conditions Eqs. (2.37) and (2.38) in Eqs. (2.41) and (2.42), $C_{1}$ and $C_{2}$ are found:

$$
\begin{gathered}
C_{1}=\frac{\tau_{s} r_{o} \ln \left(r_{*} / r_{o}\right)}{G_{m} \cosh \left(k_{D}\right)} \sinh \left(\frac{k_{D} l}{l-l_{D}}\right) \\
C_{2}=-\frac{\tau_{s} r_{o} \ln \left(r_{*} / r_{o}\right)}{G_{m} \cosh \left(k_{D}\right)} \cosh \left(\frac{k_{D} l}{l-l_{D}}\right)
\end{gathered}
$$

Substituting Eqs. (2.44) and (2.45) in Eqs. (2.40), (2.41) and (2.42) provides the following final solution to the problem: 


$$
\begin{aligned}
& W=-\frac{2 \tau_{s}\left(l-l_{D}\right)}{\lambda_{D} k_{D}^{2} E_{f} \cosh \left(k_{D}\right)} \cosh \left[k_{D}\left(1-\frac{z-l_{D}}{l-l_{D}}\right)\right] \\
& \sigma=\frac{2 \tau_{s}}{\lambda_{D} k_{D} \cosh \left(k_{D}\right)} \sinh \left[k_{D}\left(1-\frac{z-l_{D}}{l-l_{D}}\right)\right] \\
& \tau=\frac{\tau_{s}}{\cosh \left(k_{D}\right)} \cosh \left[k_{D}\left(1-\frac{z-l_{D}}{l-l_{D}}\right)\right]
\end{aligned}
$$

It follows that maximum stresses in the fiber are equal to:

$$
\sigma_{\max }=\frac{2 \tau_{s}}{\lambda_{D} k_{D}} \tanh \left(k_{D}\right) \quad \text { and } \quad \tau_{\max }=\tau_{s} \quad \text { at } z=l_{D}
$$

According to Eq. (2.46) the displacement $W$ at the ends of the fiber equals to:

$$
\begin{aligned}
& \text { for } \mathrm{z}=l_{D} \quad W_{\max }=-\frac{2 \tau_{s}\left(l-l_{D}\right)}{\lambda_{D} k_{D}^{2} E_{f}} \\
& \text { for } \mathrm{z}=1 \quad W_{\min }=-\frac{2 \tau_{s}\left(l-l_{D}\right)}{\lambda_{D} k_{D}^{2} E_{f} \cosh \left(k_{D}\right)}
\end{aligned}
$$

The length of debonding, in terms of $\sigma_{\mathrm{o}} / \tau_{\mathrm{s}^{\prime}}, \varepsilon, \mathrm{r}_{\mathrm{o}} / 1$ and $v_{\mathrm{m}^{\prime}}$ is defined by the equation:

$$
2 \tanh \left(k_{D}\right)=k_{D} \lambda_{D} \frac{\sigma_{0}}{\tau_{s}}
$$


According to Eq. (2.51), debonding proceeds, at first, in a stable manner so that $l_{D}$ grows with the increase of $\sigma_{0^{\prime}}$ after a certain maximum load is achieved debonding becomes unstable and extends over the entire interface. The maximum axial force is equal to $\tau_{s} \pi r_{o}^{2}$ multiplied by a function of $\varepsilon, \mathrm{r}_{\mathrm{o}} / l$ and $v_{\mathrm{m}}$.

The fitting index $\alpha$ defined by Eq. (2.34) can be found from the numerical or physical experiment using a requirement of coincidence or best fitting of a characteristic quantity of convenience in the problem as was explained in Section 2.2.2. As is shown in Chapter 4 the results of numerical experiments coincide very well with the approximate analytical solution Eqs. (2.46) to (2.48) if the value of $\alpha$, defined by Eq. (2.31) (Cherepanov, 1983 ), is taken.

Hence, substituting Eq. (2.31) into Eq. (2.34) and then this equation into Eq. (2.43) $\mathrm{k}_{\mathrm{D}}$ can be defined as follows:

$$
k_{D}^{2}=\frac{1.355 \varepsilon}{\lambda_{D}^{2}\left(1+v_{m}\right) \ln \left(\frac{1}{\lambda_{D}}\right)}
$$




\section{CHAPTER 3. NUMERICAL ANALYSIS}

\subsection{Introduction}

The pullout problem of a fiber embedded in a matrix under consideration is analyzed numerically by means of the finite element method (FEM). The basic concept of FEM is that the structure to be analyzed is considered to be an assemblage of discrete pieces, called elements, that are connected together at a finite number of points or nodes. Then, the structure can be analyzed in a procedure similar to that used in the beam theory when the structure has been represented by two- or three-dimensional elements. A shape function relates the displacement within the element to the displacement of the nodes. Applying equilibrium at every node, the individual element stiffness matrices are assembled into a set of linear simultaneous equations. Finally, this equation set is solved for the nodal displacements from which strains and stresses within each element can be found.

Although this method is an approximation, its validity as a convenient way of obtaining approximate solutions to variety of engineering problem has been shown widely. In addition, finite element method can be used with problems where non homogeneous, anisotropic and two or more different materials are combined. The latter fact is of special importance in the problem under study.

There are different kind of software available for the finite element analysis. For this particular case the program ANSYS version 4.4a installed in a SUN station is utilized. 
Having a brief background in what the finite element method is and which program is employed in the numerical analysis of the problem, the modeling procedure as well as the results of the numerical calculations will be discussed in the rest of the chapter.

\subsection{Modeling Procedure}

The modeling procedure corresponds to the preprocessing phase in the ANSYS program. This phase is where all relevant data, such as geometry, material properties, and loading, are defined to the database in preparation for solution. This stage is made up of the following steps: laying the foundation, where the analysis type, element type, real constants and material properties are established; building the model, where the model geometry is defined and described in terms of finite element entities (nodes and elements); specifying load data, where boundary conditions are added to the geometry to complete the model; and preparing the solution, where all the information prepared in the first three steps is extracted, filed and cast into a form suitable for the solution phase of the analysis.

Even though two problems are being considered in this work, both cases use the same model described thereupon. Nevertheless, some changes are made in the debonding problem in order to simulate the debonding process. These changes are explained in Section 3.3.2 before the results are discussed.

It is important to notice at this point that all the data in the preprocessing phase

of the ANSYS program is specified without units. However, all the data must be 
consistent in the same system of units in order to obtain a suitable solution. For instance, if all the input data is in the International System of Units (SI) (lengths in meters, forces in Newton, Young's modulus in Pascal), the results will be in the same system of units (stresses in Pascal, and displacements in meters).

Now the modeling procedure is presented following the basic steps of the preprocessing phase of the ANSYS program.

\subsubsection{Laying the Foundation}

This is the first step of the modeling process in the program. Here, the foundation is set by specifying the analysis type and establishing the element type and material properties. This work includes the analysis of different relations Young's modulus of matrix $\left(\mathrm{EX}_{\mathrm{m}}\right)$ /Young's modulus of fiber $\left(\mathrm{EX}_{\mathrm{f}}\right)$. All the relations considered in this work are:

$$
\varepsilon=\mathrm{EX}_{\mathrm{m}} / \mathrm{EX}_{\mathrm{f}}=0.1,0.2,0.01,0.001 \text {. }
$$

From the specification of the Poisson's ratio (see appendix A), it is observed that both materials have the same value for this property which is 0.3 .

According to Engineers' Guide to Composite Materials by Weeton, Peters and Thomas (1987), the Poison's ratio for crystal and glass matrix varies from $v=1 / 4$ to $1 / 2$. Due to this fact, the value of the Poisson's ratio in this work was defined as 0.3. Besides, this value is in good agreement with that used by Hsueh (1990) on interfacial debonding and fiber pullout stress of fiber-reinforced composite. II: Non-constant interfacial bond strength, where a single stainless steel wire (fiber) with material properties $E_{f}=170 \mathrm{Gpa}, v_{f}=0.35$ embedded in an epoxy resin 
matrix with properties $E_{m}=3.65 \mathrm{Gpa}, v_{m}=0.39$ is studied; with that used by Liang and Hutchinson (1992) on mechanics of the fiber pushout test, where the analysis of a fiber and outer region (matrix) using the following relation of material properties $v_{f}=v_{m}=1 / 3$ and $E_{f} / E_{m}=1$ or 3 is carried out; and finally, with that used by Cherepanov (1983) on fracture mechanics of composite materials where a carbon fiber with material properties $E_{f}=39 \times 10^{3} \mathrm{~kg} / \mathrm{mm}^{2}$ and $v_{f}=0.3$ embedded in an epoxy resin matrix with material properties $E_{m}=350 \mathrm{~kg} / \mathrm{mm}^{2}$ and $v_{m}=0.35$ is considered. See appendix A for a complete description of this first step.

\subsubsection{Building the Model}

This is the second step of the modeling process. Here, using geometry descriptions only (areas), a mathematical representation or solid model of the structure is created. Once the solid model of the structure is completed, parameters which describe the desired finite element mesh characteristic are provided. The ANSYS program then creates a finite element model, made up nodes and elements, which corresponds geometrically to the solid model.

For the analysis of this problem in the computer, a two-dimensional model is used. The design of the geometrical grid appears in Fig. 3. This geometry description of areas is obtained by means of the generation of points and lines using the $\mathrm{K}$ command for points, $\mathrm{L}$ command for lines, and $\mathrm{AL}$ command for areas in the program. In Fig. 3, $\mathrm{r}_{\mathrm{o}}$ is the radius of the fiber; $l$ is the length of the fiber; A1 represents the fiber; and A2 represents the matrix. In this work two different relations $r_{0} / l$ are considered. These relations are expressed as follow: 


$$
\lambda=\mathrm{r}_{\mathrm{o}} / l=0.1,0.01 \text {. }
$$

No small relation $\lambda$ was studied because of the numerical modeling limitation in the computer. However, the small relation studied here are in better agreement with the practical cases than that considered by Muki and Sternberg (1969) and Slaughter and Sanders (1991).

It is important to notice that from Eq. (2.6) the cylindrical perturbed domain field extends the distance $r_{x}=r \lambda^{-\alpha}$ (see Fig. 2). From Fig. 3 is observed that the total length used in the numerical model for the matrix $2 l$ is much greater than the size of the perturbed domain $r_{*}$ defined above. So that, this size of the matrix is big enough to be considered infinite modeling in this form the problem under study.

Following that, the grid element size is defined in all the points, using the ESIZE command, as follow (see Fig. 3):

for $\lambda=0.1$ the grid element size is equal to $r_{0} / 2$ at points $4,5,6$ and 7 , and it is equal to $l / 2$ at points 1,2 and 3;

for $\lambda=0.01$ the grid element size is equal to $r_{0}$ at points $4,5,6$ and 7 , and it is equal to $2 l / 3$ at points 1,2 and 3 . 


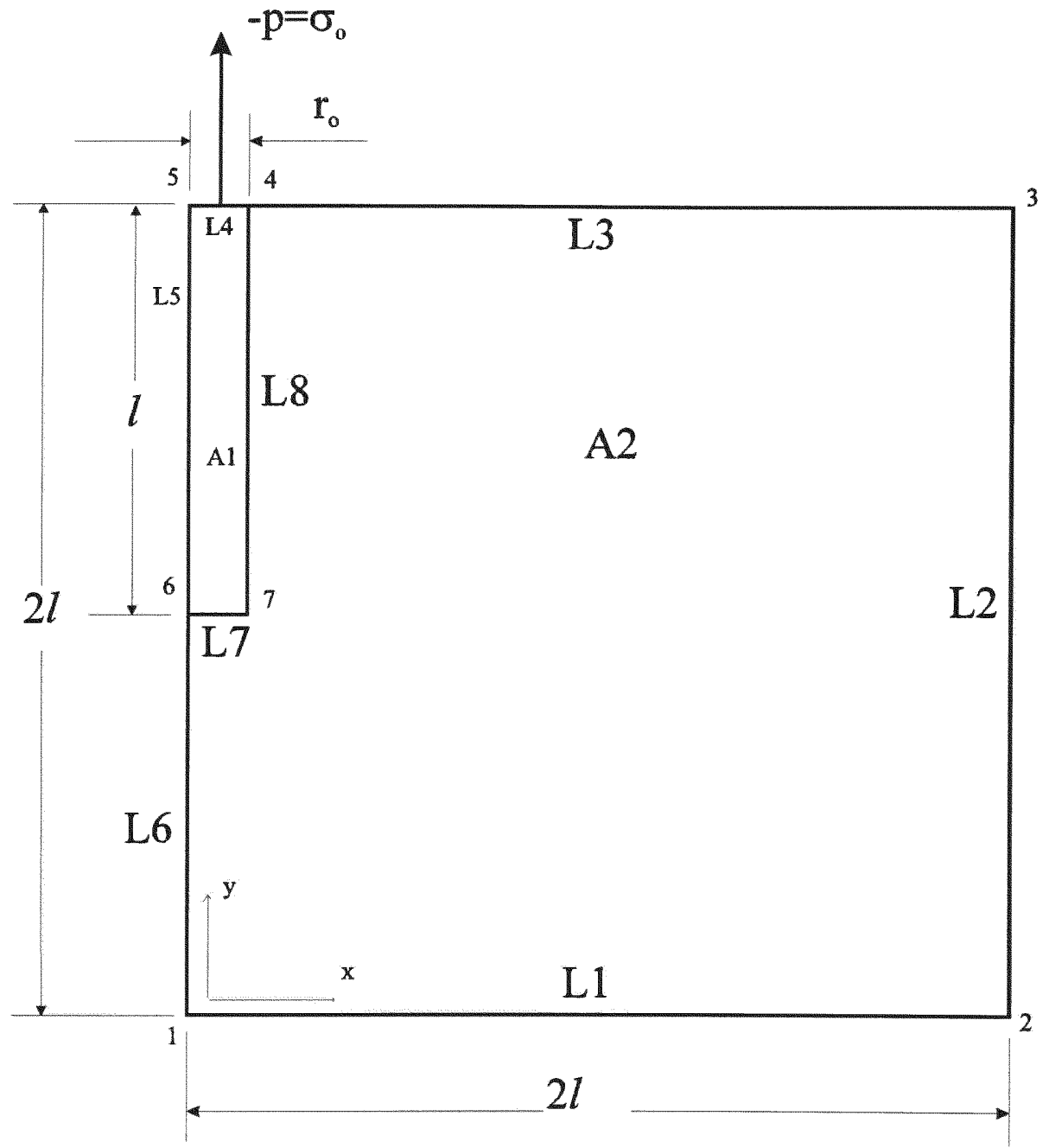

Figure 3. Geometrical design of the numerical procedure for the analysis of the fiber pullout problem using ANSYS version 4.4a. 
When the grid size element has been defined in all the points for each case, the LDVA command is used to adjust the element division controls for unmeshed lines. This means that the program automatically scales the size of the elements in the lines. Afterwards, the MESH command is used to create the mesh automatically in both areas.

Once the mesh is completed and all the nodes and elements are defined, the material properties of the fiber, which have been already defined, are assigned to the elements of area 1 (fiber); similarly, the material properties of the matrix are assigned to the elements of area 2 (matrix).

\subsubsection{Specifying the Load Data}

The third step of the modeling process corresponds to the specification of the load data. This stage refers to the configuration of boundary conditions which include constraints or support conditions, and load such as pressure.

Boundary condition constraints are placed on the model to hold it in some way. In other words, a constraint is applied when a degree of freedom is specified to remain with some given motion (in this case displacement equals to zero). All the constraints must be specified correctly, otherwise the problem is ill-posed.

For the problems under consideration, the constraints are defined using first the NSEL command to select the nodes along the line whose motion will be restricted of movement, and then using the UX, UY, and/or UZ command to 
specified the direction of the restriction. The following constraints are specified (see Fig. 3):

for $\mathrm{y}=0$ (line L1) $\quad \mathrm{UY}=0, \quad \mathrm{UX}=0$;

for $\mathrm{x}=0$ (line L5 and L6) $\quad \mathrm{UX}=0$;

for $\mathrm{x}=2 l$ (line L2) $\quad \mathrm{UY}=0, \quad \mathrm{UX}=0$;

the constraint for all grid nodes $\mathrm{UZ}=0$ is understood by the program due to the condition of axisymmetry defined in the element selected (Section 3.2.1).

Load boundary conditions include concentrated and/or distributed loads. For the case of the elastic pullout problem, a unique load is applied at the top of the fiber (line L4 in Fig. 3) using the LPSF command in the following way:

$$
\text { LPSF, 4, }-100 \text {, }
$$

which means that a load of -100 units of pressure is applied in line LA (top of the fiber). The minus sign means that this is a traction load. For the case of the debonding problem, different loads are applied using the same command. These values are discussed in Section 3.3.2.

\subsubsection{Preparing the Solution}

This is the last step of the modeling process in the computer. Here, all the data is written into a file in a suitable form in order to be read and understood by the solution phase of the program. First, the WSORT command is used to reorder the elements for smaller wave front (number of linear simultaneous equations to be solved). The first calculations of this work (elastic pullout problem) were done using the program with a maximum capacity of 200 wave front. After increasing the wave front capacity to 800, the last calculations (debonding 
problem) were executed. However, for both cases the wave front of the model under study remains under 200 after the reorder instruction. Then, the command AFWRITE is used to write a coded file which is needed for input to the solution phase.

\subsection{Numerical Solution}

After the preprocessing phase is finished, the command /INPUT,27 is used to proceed with the solution phase. Here, the program solves the problem and sends the output data to a file. The post processing stage follows to the solution phase. In this last phase of the whole numerical procedure, the results are read from the postdata file by means of the /POST1 and SET commands. Next, the command PRNSTR, COMP is used to display the components of the stresses for each node which are the numerical results of the problem. Finally, the maximum displacement is obtained using the PRDISP, UY command which gives the displacements of the nodes in $Y$ direction.

The results are based on the average nodal stresses. $\sigma_{\mathrm{z}}$ is considered as the average value of the stresses between the nodes in the same cross-section of the fiber. $\tau_{\mathrm{rz}}$ is considered as the shear stress of the nodes along the lateral line of the fiber (line L8 in Fig. 3). $W_{\max }$ is considered as the maximum displacement of the fiber at the top (line L4 in Fig. 3).

It is important to note that although $x-y$ coordinate system with origin at the bottom of the matrix is used in the ANSYS program model (Fig. 3), the appropriate z-r coordinate system with origin at the top of the fiber is used from 
now on to present all the results. Consequently, the length of the fiber varies from $\mathrm{z}=0$ to $\mathrm{z}=l$, and the radius of the fiber varies from $\mathrm{r}=0$ to $\mathrm{r}=r_{0^{\circ}}$.

\subsubsection{Results of the Elastic Pullout Problem}

In this section the numerical results of the elastic pullout problem are presented. For this purpose, convenient dimensionless tables showing the values of $\sigma_{z} / \sigma_{\mathrm{o}}$ and $\tau_{\mathrm{rz}} / \sigma_{\mathrm{o}}$ for the total length of the fiber with increments of $0.05 \mathrm{in} \mathrm{z} / \mathrm{l}$ have been designed. The values of $\sigma_{\mathrm{z}}$ and $\tau_{\mathrm{rz}}$ are taken from the output data file in the post processing phase of the numerical calculations in the computer as was explained previously, and $\sigma_{\mathrm{o}}$ is the value of the nominal normal stress applied at the top of the fiber. The advantage of presenting the results using dimensionless quantities is that in this form the results are independent of the system of units chosen for the numerical analysis.

Tables 1 and 2 present the results of the normal stress for the cases $\lambda=0.1, \varepsilon=0.1$ and $\lambda=0.1, \varepsilon=0.2$ respectively. The results of the normal stress for the cases for $\lambda$ $=0.01$ and $\varepsilon=0.1,0.01$, and 0.001 are given in Tables 3,4 , and 5 respectively. The

results of the shear stress for all the cases considered before are given in Tables 6 to 10 (see appendix B).

The maximum displacement for all the relations of $\lambda$ and $\varepsilon$ considered in the numerical analysis of the elastic pullout problem is presented in Table 11 (see appendix B) using an appropriate dimensionless quantity. 


\subsubsection{Results of the Debonding Problem by Pullout}

For the analysis of the debonding problem of a fiber embedded in a matrix by pullout, the same model explained in Section 3.3.1 and employed for the analysis of the elastic pullout problem is used; however, a change is introduced to the model in order to simulate the debonding process in the computer. From the numerical results of the elastic pullout problem, which is a necessary prelude to fiber debonding, presented in Section 3.3.2, it is expected that the maximum shear stress occurs on the interface at the top of the fiber (see Cherepanov and Esparragoza, 1992). Due to this fact, the debonding process starts at the free boundary of the matrix half-space and grows along the interface. In order to model this physical behavior in the computer, different traction loads are applied at the top of the fiber until the first node in the interface reaches the value of $\tau_{\mathrm{s}}$, which is the limiting shear stress. In the numerical analysis a value of $\tau_{\mathrm{s}}=0.001 E_{m}$ is assumed. Then, the Young's modulus of the elements of the matrix on the interface corresponding to $l_{D}$ are changed to a very small value $\left(\mathrm{E}=0.00001 E_{m}\right)$ modeling in this way the open bond of length $l_{D}$ in the interface between the fiber and the matrix (See Fig. 4). A special numerical experiment was conducted decreasing the Young's modulus of interface elements until this value $\left(E=0.00001 E_{m}\right)$ stopped influencing the solution, which meant that the practical zero necessary to model the interface opening was achieved. After that a new load is applied at the top of the fiber until the first node corresponding to $z=l_{D}$ reaches $\tau_{\mathrm{s}}$. All the procedure is repeated for different increased values of $l_{D}$ until $l_{D}=l$. 
All the numerical results related to the debonding problem are presented in appendix $C$. Here, the meaning of the parameters $\sigma_{z^{\prime}}$ and $\tau_{\mathrm{rz}}$ is the same explained in Section 3.3.2. Tables 12 and 13 present the relation between the load applied and the length of debonding for the cases of $\lambda=0.1$ and $\varepsilon=0.1$ and 0.2 respectively using the dimensionless quantities $l_{D} / l$ and $\sigma_{o} / \tau_{s}$. The same relation for the cases of $\lambda=0.01$ and $\varepsilon=0.1,0.01$, and 0.001 is shown in Tables 14, 15, and 16 respectively. The distribution of the normal stress along the fiber for all the relations $\lambda$ and $\varepsilon$ studied for the case of the length of debonding $l_{D} / l=0.1$ are presented in Tables 17 to 21 using the dimensionless quantities $z / l, \sigma_{z} / \tau_{\mathrm{s}}$. The distribution of the shear stress along the interface for all the relations studied are given in Tables 22 to 26 using the dimensionless parameters $\mathrm{z} / l$ and $\tau_{\mathrm{rz}} / \tau_{\mathrm{s}}$. 


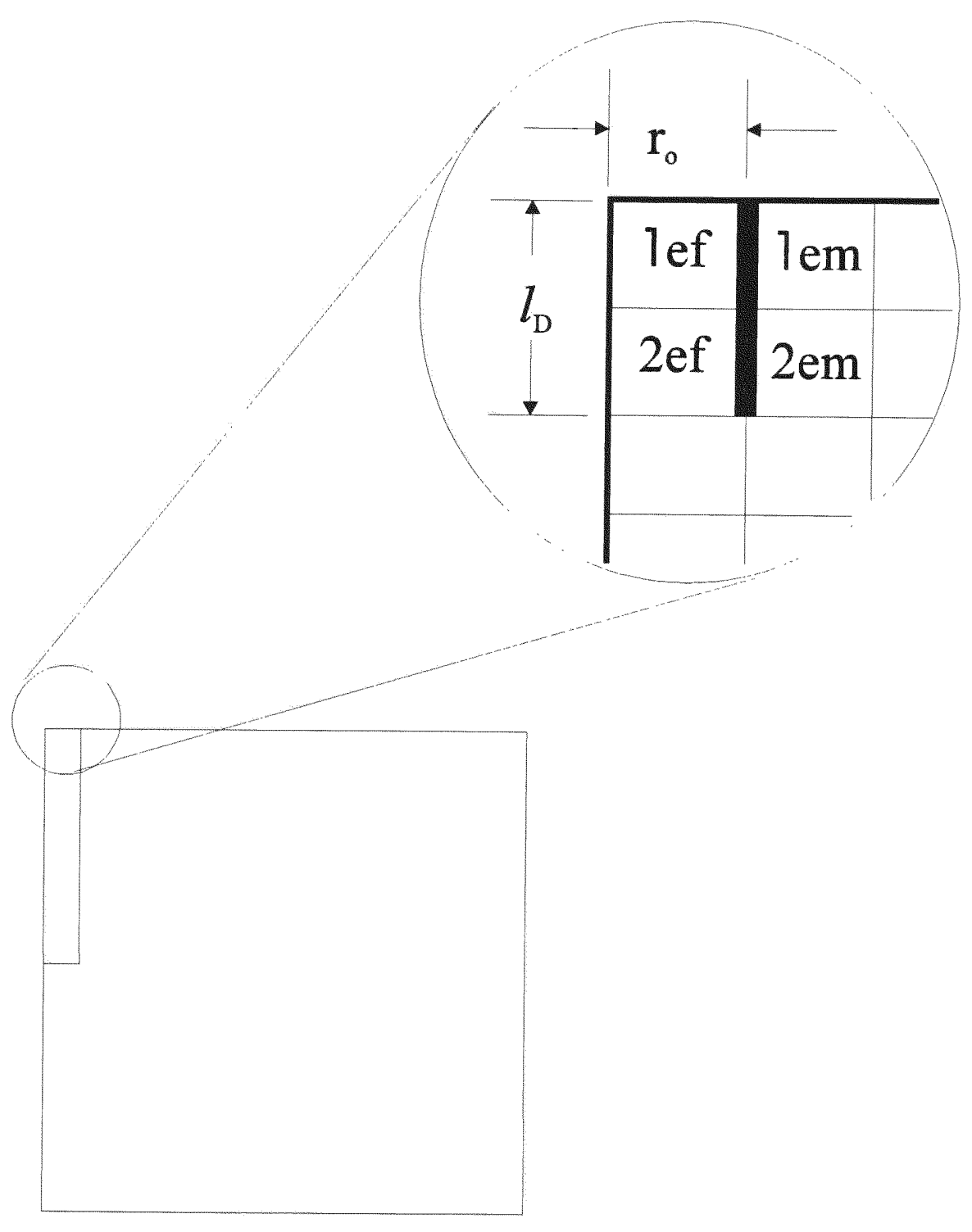

Figure 4. Detail of the local debonding zone. Here, 1em and 2em are the elements corresponding to $l_{D}$ whose Young's modulus was changed in order to model the debonding process in the finite element analysis using the program ANSYS version 4.4a. 


\section{CHAPTER 4. COMPARISON OF NUMERICAL AND ANALYTICAL RESULTS}

\subsection{Introduction}

In the two previous Chapters, two different approaches were presented in order to find a solution to the pullout problem including its two stages: before and after debonding occurs. Chapter 2 dealt with an approximate analytical solution for this problem based on principles and assumptions explained before taking into account small relations $\lambda=r_{o} / l$ and $\varepsilon=E_{m} / E_{f}$. This theoretical approach is different from those suggested by other authors such as Cox, Kelly, Muki et al., Pak and more recently Hsueh, and Slaughter and Sanders. On the other hand, in Chapter 3 the same problem including both its stages is analyzed numerically by means of the finite element method. In this Chapter, these two different approaches to the same problem are compared. Due to the fact that there is no experimental data available for this problem, this comparison is important because it establishes the coincidence of a new analytical solution and the numerical solution. Also, it helps in understanding the problem under study, and to set the discrepancies between both approaches and their possible reasons.

The comparison is based on the distribution of stresses $\sigma_{\mathrm{z}}$ and $\tau_{\mathrm{rz}}$ along the fiber basically. However, for the particular problem under study (elastic pullout problem or debonding problem by pullout) another parameter of interest will be compared. For this purpose, graphs and tables with dimensionless quantities are used. These graphs and tables will be explained in Section 4.2 for the elastic pullout problem and in Section 4.3 for the debonding problem by pullout. 


\subsection{Comparison of the Elastic Pullout Problem Results}

In this Section, the analytical and numerical results of the elastic pullout problem are compared. For this purpose, two graphs with dimensionless values of $\sigma_{z} / \sigma_{\mathrm{o}}$ vs. $\mathrm{z} / l$ and $\tau_{\mathrm{rz}} / \sigma_{\mathrm{o}}$ vs. $\mathrm{z} / l$ were designed. The numerical values of $\sigma_{\mathrm{z}} / \sigma_{\mathrm{o}}$ and $\tau_{\mathrm{rz}} /$ $\sigma_{\mathrm{o}}$ are taken from the computer results as was explained in Section 3.3.2. These values are compiled in Tables 1 to 10 . The analytical values of $\sigma_{z} / \sigma_{\mathrm{o}}$ and $\tau_{\mathrm{rz}} / \sigma_{\mathrm{o}}$ are calculated using Eqs. (2.24) and (2.25) respectively, taking a fitting index $\alpha$ $=0.738$ suggested in Section 2.2.2 (see appendix B Tables 1 to 10 ).

The results of the analytical and numerical calculations for each one of the different relations of the dimensionless parameters $\lambda(0.1$ and 0.01$)$ and $\varepsilon(0.1$, $0.2,0.01$ and 0.001 ) considered in this study are plotted in a graph to compare both methods (See Figs. 5 to 14). These relations were used because they are of practical importance in composites. No small geometrical relation $\lambda$ was considered due to the modeling limitation in the computer program. Generally speaking, it can be observed that both approaches tend to show very close behavior graphically with small discrepancies in general although substantial differences can be found at particular points.

The graphic behavior of both methods studied in this work is in good agreement with that found by Muki and Sternberg (1969) and Slaughter and Sanders (1991) who studied a similar problem but each using a different approach respectively. 
In the analysis of this problem, another relation including the maximum displacement is considered for comparison. This comparison is presented in Table 27 in Appendix D.

The analysis for the normal stresses, shear stresses and displacement is presented as follows.

\subsubsection{Comparison of Normal Stresses}

Figs. 5 and 7 present the relation $\sigma_{z} / \sigma_{o}$ vs. $z / l$ for the case of $\lambda=0.1$, and $\varepsilon=0.1$ and 0.2 respectively. Figs. 9, 11, and 13 correspond to the same relation for the cases $\lambda=0.01$, and $\varepsilon=0.1,0.01$, and 0.001 .

There is a point of interest related to the nature of the problem which is important to note in Figs. 5, 7, 9, 11, and 13. Here, the normal stress decrease from a maximum value $\left(\sigma_{z} / \sigma_{0}=1\right)$, which corresponds to the load applied, to a minimum value very close to zero, which is one of the assumptions established in the boundary conditions in the analytical solution. It is observed, too, that this decrease depends on the dimensionless relations $\lambda$ and $\varepsilon$. For the case of $\lambda=0.01$ and $\varepsilon=0.1$, the normal stress decrease rapidly, so that its influence is felt only in $1 / 4$ of the total length of the fiber (See Fig. 9). This is expected because a very thin fiber embedded in a considerably stiffer matrix is studied. Due to this fact, the stress transfer between fiber and matrix through the interface occurs in the first $1 / 4$ of the total embedded length; after this point, practically no stress transfer occurs. When $\lambda=0.01$ and $\varepsilon=0.01$, the decrease of the normal stress is not as fast as the above case, but, it is faster than the other cases, and its influence is 
important until approximately $3 / 4$ of the total length (See Fig. 11). The other three cases considered show no great difference of this decrease between them (See Figs. 5, 7, and 13). From this analysis, it can be concluded that the stress transfer behavior between fiber and matrix depends on the size of the fiber determined by the parameter $\lambda$ and the material properties of the components of the composites established by the relation $\varepsilon$.

Comparing the normal stress results of the analytical and numerical results, the following features can be observed: a) For all the relations $\lambda$ and $\varepsilon$ studied, the difference among both approaches appears very small for $z / l<0.5$ (except for $z / l$ near to zero), and tends to increase from this point to $\mathrm{z} / \mathrm{l}=1$. The difference of both curves, analytical and numerical, at the top of the fiber can be explained by the local effects which are physically impossible to avoid. A numerical experiment was carried out extending the fiber the distance $2 r_{0}$ from the free boundary surface of the matrix and applying the load at the top of the fiber. For this case the discrepancy at $z / l=0$ decreases slightly. This behavior explain the local effect and the intuitive idea of the Saint Venant's principle. However, the difference between both methods, analytical and numerical, at $z=0$ using the original model discussed is small enough to consider this model suitable to be used. b) Although both approaches for all the cases considered show very close behavior, the case of $\lambda=0.01$ and $\varepsilon=0.01$ show the closest correlation between both solutions (See Fig. 9). Consequently, it can be concluded that the coincidence of both solutions depends on the combination of the dimensionless parameters $\lambda$ and $\varepsilon$. 


\subsubsection{Comparison of Shear Stresses}

Figs. 6 and 8 present the relation $\tau_{\mathrm{rz}} / \sigma_{\mathrm{o}}$ vs. $\mathrm{z} / l$ for the case of $\lambda=0.1$, and $\varepsilon=0.1$ and 0.2 respectively. The same relation for the cases $\lambda=0.01$, and $\varepsilon=0.1,0.01$, and 0.001 are shown in Figs. 10, 12, and 14.

Due to the close relation between the normal stress in the fiber and the shear stress at the interface, the graphs $\tau_{\mathrm{rz}} / \sigma_{\mathrm{o}}$ vs. $z / l$ greatly resembles the graph $\sigma_{z} / \sigma_{\mathrm{o}}$ vs. $z / l$. Just as the normal stress decreases from a maximum value to a minimum value, so the shear stress decreases from a maximum value at the top of the interface to a minimum value at the end of the interface; besides, this decrease also depends on the relations $\lambda$ and $\varepsilon$. Here, for the case of $\lambda=0.01$ and $\varepsilon=0.1$, the shear stress decrease rapidly and its influence is considerable only in $1 / 4$ of the total length of the interface (See Fig. 10).

Considering the same relation $\lambda$ and the same load $\sigma_{0}$, it can be seen that while the relation $\varepsilon$ decreases so does the maximum shear stress developed at the interface. As a result, it is expected that the shear stress developed at the interface reaches the limiting shear stress faster while $\varepsilon$ increases for the same relation $\lambda$.

Comparing the shear stress results of the analytical and numerical results, the following characteristics can be observed: a) For the cases $\lambda=0.1$, and $\varepsilon=0.1$ and 0.2 (See Figs. 6 and 8), the numerical curve of $\tau_{\mathrm{rz}} / \sigma_{\mathrm{o}}$ has a maximum peak at $z / l=0.05$. This can be explained not only by the local effect but also for the nature of the mathematical solution. The point $\mathrm{z} / l=0$ is a singular point; so that, the 
shear stress at this point tends to infinity but the program solves for a finite value showing this peak close to $\mathrm{z} / \mathrm{l}=0$ (see section 4.3 .3 for more details about the singularity). b) Contrary to the above cases, when $\lambda=0.01$, and $\varepsilon=0.1,0.01$, and 0.001 , the numerical curve of $\tau_{\mathrm{rz}} / \sigma_{\mathrm{o}}$ is smooth having no peak value. For these cases, the grid element size is smaller than that of the previous cases, which permits to have more nodes along the interface and, hence, to show this smooth result. c) Like in the normal stress, both approaches for all the cases considered show practically the same behavior; nevertheless, the case of $\lambda=0.01$ and $\varepsilon=0.01$ shows the closest correlation between both solutions.

\subsubsection{Comparison of Displacements}

Another relation that can be used in order to compare the analytical and numerical results is one which includes the displacements and is called the bed coefficient $\left(\mathrm{B}=\mathrm{F} / W_{\max }\right)$. In order to present these results using a dimensionless parameter, the following relation is defined:

$$
\bar{B}=\frac{B}{r_{o} E_{f}}
$$

So that, from Eqs. (2.29) and (2.30) it follows:

$$
\bar{B}_{\text {analytic }}=\pi \lambda k \tanh (k),
$$

and from Eqs. (2.13) and (2.29) it follows:

$$
\bar{B}_{\text {numeric }}=\frac{\pi \sigma_{o} r_{o}}{E_{f} W_{\max }}
$$

where $W_{\max }$ is taken from Table 11. 
These results are shown in appendix D Table 27 . When both analytical and numerical results are compared, it can be shown that the difference appears small in the general case, and the behavior of the results is the same. This bed coefficient represents the slope of the line $\mathrm{F}=\mathrm{B} W_{\max }$. While the composite works in the elastic range this value must remain constant. A variation of this coefficient means that the plasticity range or the limiting shear stress at the interface has been reached.

\subsection{Comparison of the Debonding Problem by Pullout}

Here, the numerical and analytical results of the debonding problem by pullout are compared. Similar to the comparison of the elastic pullout problem, in this section three types of graphs with dimensionless parameters are used. The first type uses the dimensionless quantities $\sigma_{\mathrm{o}} / \tau_{\mathrm{s}}$ and $l_{D} / l$, and shows the relation between the load applied and the length of debonding (See Figs. 15 to 19). The numerical values of $\sigma_{\mathrm{o}} / \tau_{\mathrm{s}}$ for all the cases $\lambda$ and $\varepsilon$ considered is found in Tables 12 to 16 , and the analytical values are calculated using Eq. (2.51) (see appendix C Tables 12 to 16). The second type of graphs uses the relations $\sigma_{\mathrm{z}} / \tau_{\mathrm{s}}$ and $\mathrm{z} / l$, which exhibits the normal stress distribution along the fiber. The third type uses the relations $\tau_{\mathrm{rz}} / \tau_{\mathrm{s}}$ and $\mathrm{z} / \mathrm{l}$ showing the shear stress distribution along the interface. The last two types of graphs correspond to a particular length of debonding. The particular length of debonding considered in this work in order to compare both solutions is $l_{D} / l=0.1$ (See Figs. 20 to 29). Tables 22 to 26 bring the numerical values for all the cases studied, and the analytical values of $\sigma_{z} / \tau_{s}$ and $\tau_{\mathrm{rz}} / \tau_{\mathrm{s}}$ are calculated using Eqs. (2.47) and (2.48) respectively taking a fitting index $\alpha=0.738$ (see Tables 22 to 26). 
The graph used to compare the shear stress considering a particular length of debonding are in good agreement with that developed by Majudmar et al. (1993) who analyzed the pushout tests on an SiC-fiber-reinforced reaction-bonded $\mathrm{Si}_{3} \mathrm{~N}_{4}$ composite on the basis of a model using shear-lag theory applied to a partially debonded composite.

The comparison of the debonding problem by pullout is very much like the comparison of the elastic pullout problem explained before in that they both present the same elements of comparison and the behavior of the solutions have the same form. Here likewise, both approaches tend to show very close behavior with small discrepancies in general; however, substantial differences can be found at particular points.

The comparison of the relation between the load applied and the length of debonding, as well as the comparison of the distribution of normal and shear stress for this problem are presented as follows.

\subsubsection{Comparison of the Relation between the Load Applied and the Length of Debonding}

Figures 15 and 16 establish the relation between $\sigma_{\mathrm{o}} / \tau_{\mathrm{s}}$ and $l_{D} / l$ for the cases of $\lambda$ $=0.1$, and $\varepsilon=0.1$ and 0.2 respectively. The same relation for the case $\lambda=0.01$, and $\varepsilon$ $=0.1,0.01$ and 0.001 is set in Figs. 17, 18, and 19 .

From these figures, it is noticed that the numerical and analytical results present the same behavior for the different combinations of parameters $\lambda$ and $\varepsilon$ studied. 
The discrepancy between both approaches appears very small for $l_{D}$ less than $60 \%$ of the total length of the fiber, but, a considerable increase of this discrepancy is observed after this point for all the cases considered except for the case of $\lambda=0.01$ and $\varepsilon=0.001$ where the small discrepancy remains along the total length of the fiber (Fig. 19). The discrepancy for the cases $\lambda=0.1$ and $\varepsilon=0.1$ and $\varepsilon$ $=0.2$ reaches the maximum value at $l_{D} \sim 85 \%$ of the length $l$. These differences between both approaches are $42 \%$ and $47 \%$, respectively. For the cases of $\lambda=0.01$ and $\varepsilon=0.1$ and $\varepsilon=0.01$ the discrepancies between both methods are $46 \%$ and $36 \%$ at $l_{D} \sim 95 \%$ of the length $l$, respectively.

From the above analysis, it can be seen that the comparison between this two approaches is reliable for the case of $l_{D}$ less than $60 \%$ of $l$. After this point, the discrepancy is large enough to consider the comparison unreliable. This discrepancy can be explained from the nature of the analytical solution. The analytical approach is based on small relations $\lambda$ and $\varepsilon$. For the case of the debonding problem the dimensionless parameter $\lambda$ is defined as $\lambda_{D}=r_{0} / l-l_{D}$, where $l-l_{D}$ corresponds to the effective embedded length. While $l_{D}$ grows along the interface, the relation $\lambda_{\mathrm{D}}$ increases because the effective embedded length decreases. So that, the original assumption of $\lambda<<1$ is violated. It is seen for the cases analyzed that when $\lambda_{D}$ reaches the value of 1 ( at $l_{D} / l=0.9$ for $\lambda=0.1$ and $l_{D} / l=1$ for the case of $\lambda=0.01$ ) no analytical solution can be found using the analytical approach described in Chapter 2.

For $\lambda=0.1$, and $\varepsilon=0.1$ and $\varepsilon=0.2$ as well as for $\lambda=0.01$ and $\varepsilon=0.01$ (Figs. 15, 16 and 18), the numerical calculations show a peak at approximately $5 \%$ of the total length. This can be explained taking into account what was explained in Section 
4.2.2 about the singular point and the evaluation of the maximum value of shear stress. It is important also to consider at this point not only the above reason but also the modeling process of this problem explained in Chapter 3, and particularly in Section 3.3.3.

For the cases $\lambda=0.01$, and $\varepsilon=0.1$ and $\varepsilon=0.01$ (Figs. 17 and 18), the numerical solution is constant from $10 \%$ to approximately $90 \%$ and $75 \%$ of the total length, respectively. These solutions resemble the analytical solutions in that the analytical curves decrease slightly until the same percentages and then drop rapidly.

From these graphs (Figs. 15 to 19), it can be concluded that there is no stable growth of $l_{D}$. This means that $\sigma_{\mathrm{o}}$ reaches $\sigma_{\max }$ when $\tau_{\mathrm{rz}}$ reaches $\tau_{\mathrm{s}}$ at $\mathrm{z}=0$. After that, debonding becomes unstable and extends on the entire interface.

\subsubsection{Comparison of Normal Stresses}

Figs. 20 and 22 present the results of $\sigma_{z} / \tau_{s}$ vs. $z / l$ for the cases $\lambda=0.1, \varepsilon=0.1$ and 0.2 , and $l_{D} / l=0.1$ respectively. Figures 24,26 and 28 show the same results for $\lambda$ $=0.01, \varepsilon=0.1,0.01$ and $\varepsilon=0.001$, and $l_{D} / l=0.1$.

From these graphs (Figs. 20, 22, 24, 26, and 28), it is observed that $\sigma_{\mathrm{z}} / \tau_{\mathrm{s}}$ remains constant from 0 to $l_{D} / l=0.1$, which is expected because of the open bond in the interface of length equal to $l_{D}$, so the condition of no-traction holds over the open bond. These graphs have the same form from $\mathrm{z} / l=0.1$ to $\mathrm{z} / l=1$ as the elastic pullout graphs shown in (Figs. 5, 7, 9, 11 and 13) which means that the open 
bond at the interface of length $l_{D}$ has been modeled adequately. Here, it can be seen that the local effect noticed in Figs. 5 and 7 is not noticed in these graphs at $z / l>0.1$. This is expected because the load is applied at the top of the fiber at certain distance $z / l=0.1$ considered large enough to diminish the local effect of the load at the debond zone tip.

From Figs. 20, 22, 24, 26 and 28 it is noticed that when the relation $\varepsilon$ decreases the maximum value of the relation $\sigma_{z} / \tau_{\mathrm{s}}$ increases for the same relation of $\lambda$. This means that considering the same value of the limiting shear stress when $\varepsilon$ decreases for the same relation of $\lambda$ a larger load is necessary in order to force the bond to break at the interface. This analysis permits us to establish the best combination of components for the fiber-reinforced composites knowing the working load.

Similar to the previous comparison, both approaches show the same behavior, and all the characteristics noticed in the analysis of the normal stress in the elastic pullout problem are observed here. Again, the discrepancies between both methods can be explained using the arguments given previously (see section 4.2.1).

\subsubsection{Comparison of Shear Stresses}

Figs. $21,23,25,27$ and 29 show the relation $\tau_{\mathrm{rz}} / \tau_{\mathrm{s}} \mathrm{vs} . \mathrm{z} / l$ for $l_{D} / l=0.1$, for all the relations $\lambda$ and $\varepsilon$ studied. 
From theses graphs, it is observed that from 0 to $l_{D} / l=0.1, \tau_{\mathrm{rz}} / \tau_{\mathrm{s}}$ is zero for the analytical solution and practically zero for the numerical solution in all the cases. Similar to the normal stress analysis, this is due to the open bond in the interface of length equal to $l_{D}$, the condition of no-traction holds over the open bond in the interface. After this point, these figures resemble Figs. 6, 8, 10, 12 and 14 of the elastic pullout problem. All the features analyzed for the shear stress in the elastic pullout problem are practically the same as those found here (see section 4.2.2). However, the role of the singularity at the debond zone tip merits an analysis in order to understand and clarify the treatment of this point in this work.

\section{a. Singularity at the debond zone tip}

Here, it is important to notice a particular feature related to the debond zone tip. The graphs used to compare the shear stress show a steep increase in shear stress at $\mathrm{z}=l_{D} / l=0.1$. Although the peak shear stress values predicted by both methods almost coincide, the location of the peak shear stress as computed by the finite element method occurs slightly ahead of the debond zone tip $\left(z=l_{D} / l=0.1\right)$. Note that away from the debond zone tip, the analytical model solution shows a very close coincidence with the numerical results. The large and possibly singular interfacial shear stress at $z=l_{D} / l=0.1$ computed by the finite element method is not predicted by the model. Any approximate model including that of Muki and Sternberg (1970), and that of Slaughter and Sanders (1991) show certain inaccuracy near singularities. However, this does not invalidate their usefulness in fracture mechanics applications where path independent integrals play a 
decisive role. For this reason, stability of the debonded zone may be more fruitfully analyzed by a compliance method based on energy analysis.

The ANSYS program has several element types available for modeling the singular behavior at a crack tip. These elements are used in the analysis of crack for determining the displacements and stress field in the neighborhood of the tip crack. This information is useful in this case for the calculation of the stress intensity factor. Nevertheless, the stresses found using the singular element, which are an approximation because of the modeling nature, are considerable large. No attempt was made in this work to study the nature of the singular stress field at the debond zone tip by a more advanced finite element analysis. Additionally, the numerical procedure and the treatment of this singular point in this work are in good agreement with the work of Majudmar, Singh and Singh (1992) on the analysis of pushout tests on an SiC-fiber reinforced reactionbonded $\mathrm{Si}_{3} \mathrm{~N}_{4}$ composite. 


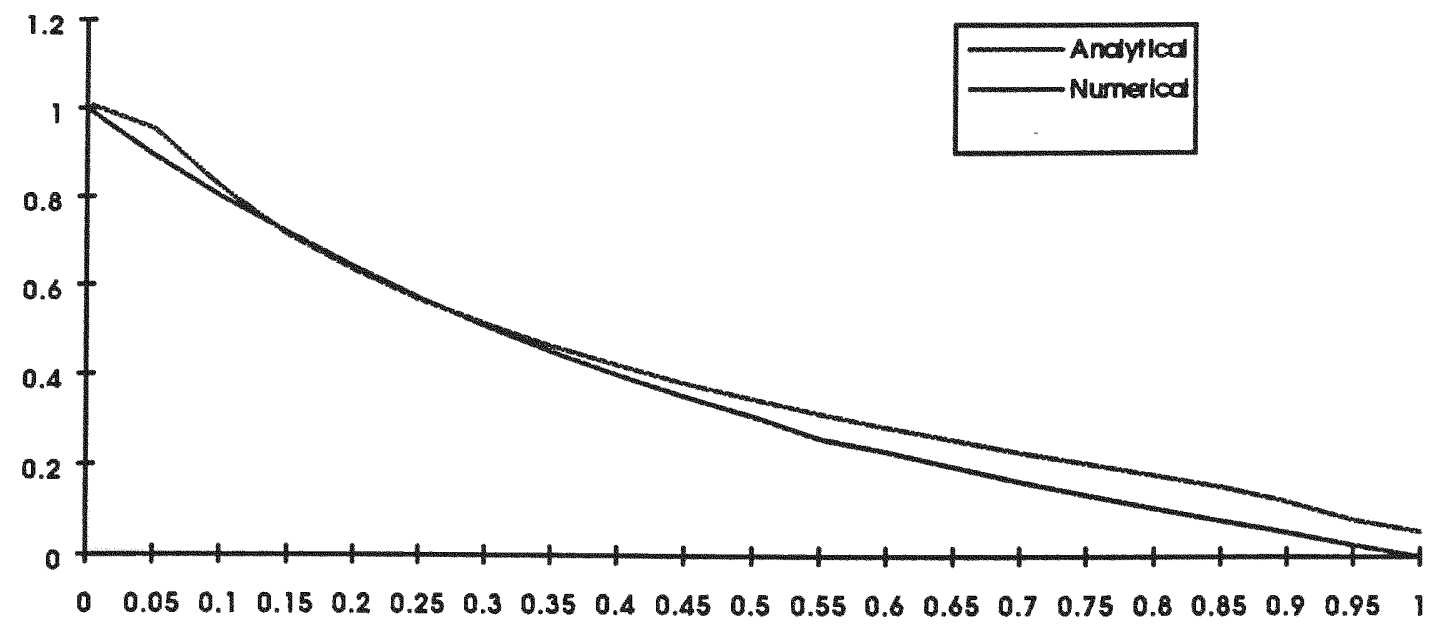

Figure 5. Graph of $\sigma_{z} / \sigma_{0}$ vs. $z / l$ for $\lambda=0.1$ and $\varepsilon=0.1$.

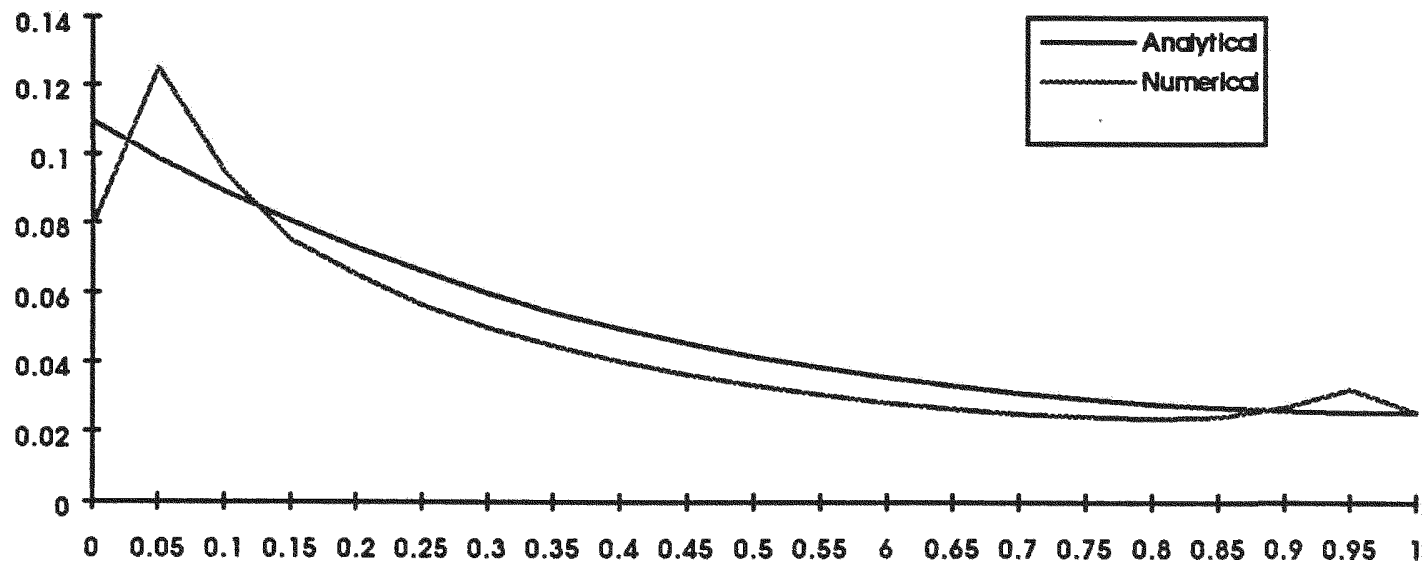

Figure 6. Graph of $\tau_{\mathrm{rz}} / \sigma_{\mathrm{o}}$ vs. $\mathrm{z} / \mathrm{l}$ for $\lambda=0.1$ and $\varepsilon=0.1$ 


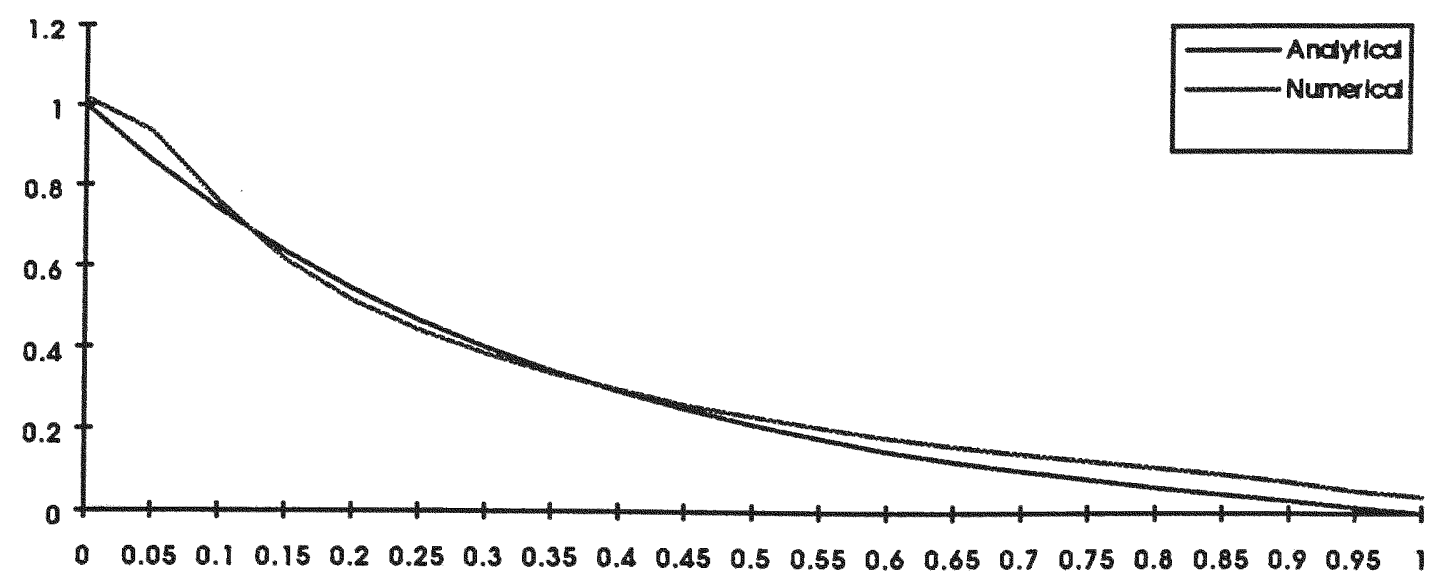

Figure 7. Graph of $\sigma_{z} / \sigma_{o}$ vs. $z / l$ for $\lambda=0.1$ and $\varepsilon=0.2$.

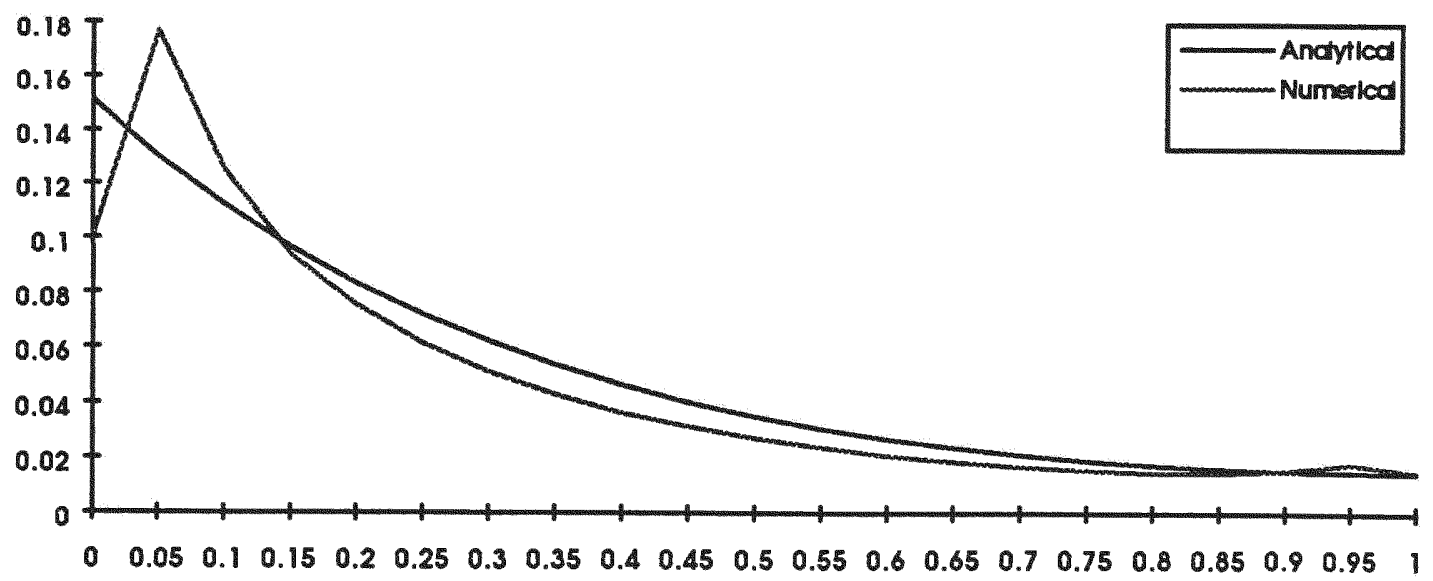

Figure 8. Graph of $\tau_{\mathrm{rz}} / \sigma_{\mathrm{o}}$ vs. $z / l$ for $\lambda=0.1$ and $\varepsilon=0.2$ 


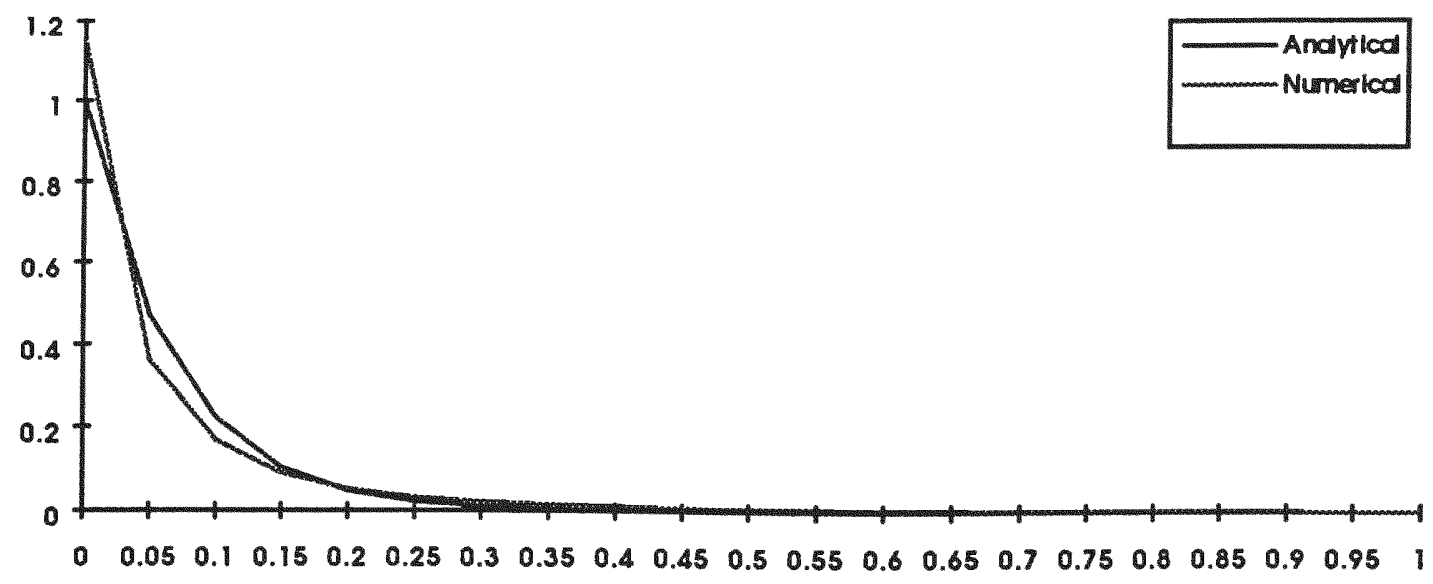

Figure 9. Graph of $\sigma_{z} / \sigma_{0}$ vs. $z / l$ for $\lambda=0.01$ and $\varepsilon=0.1$

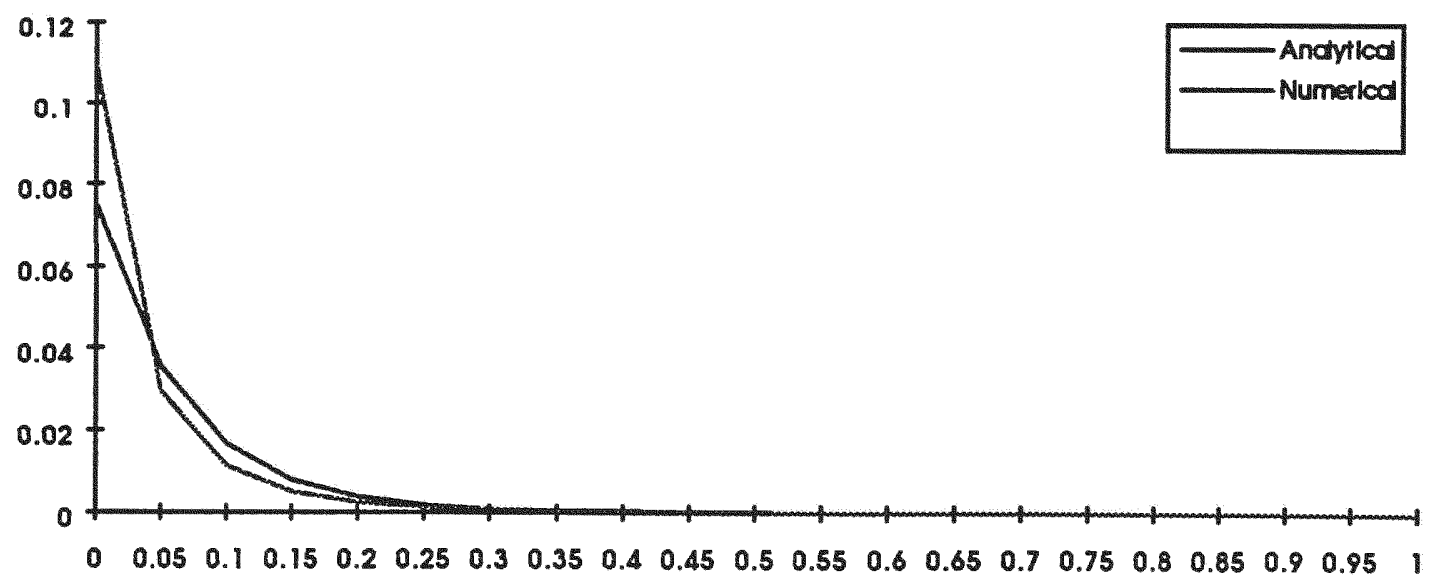

Figure 10. Graph of $\tau_{\mathrm{rz}} / \sigma_{\mathrm{o}}$ vs. $z / l$ for $\lambda=0.01$ and $\varepsilon=0.1$ 


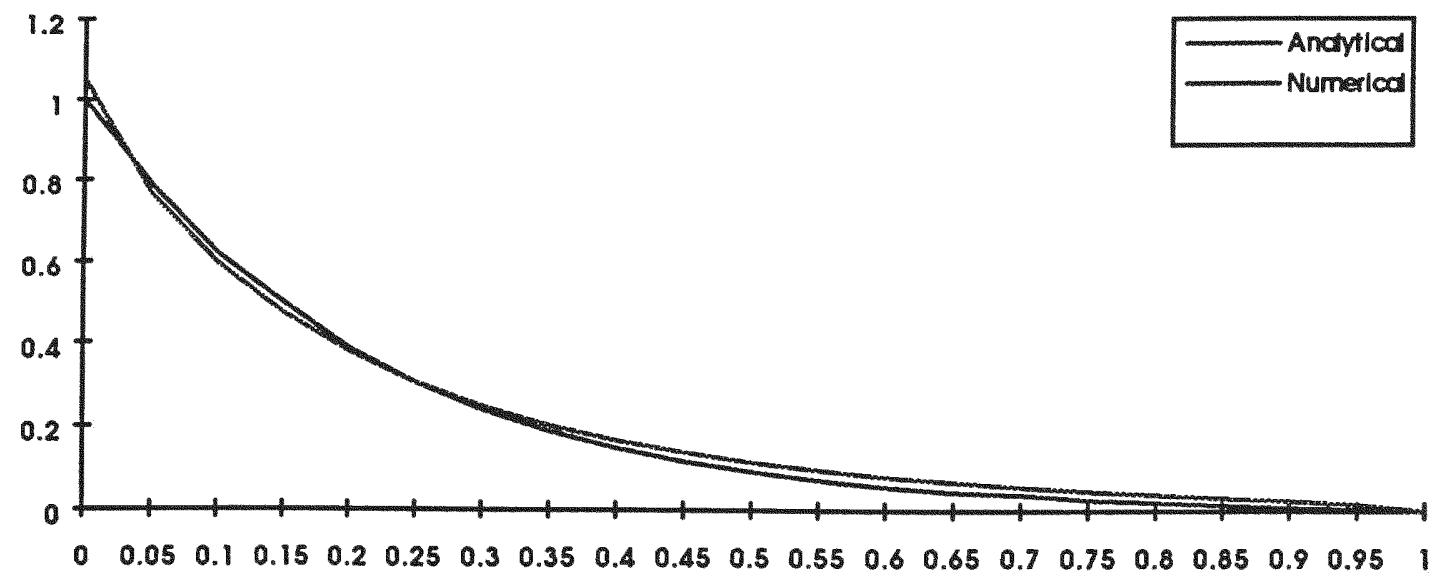

Figure 11. Graph of $\sigma_{z} / \sigma_{0}$ vs. $z / l$ for $\lambda=0.01$ and $\varepsilon=0.01$

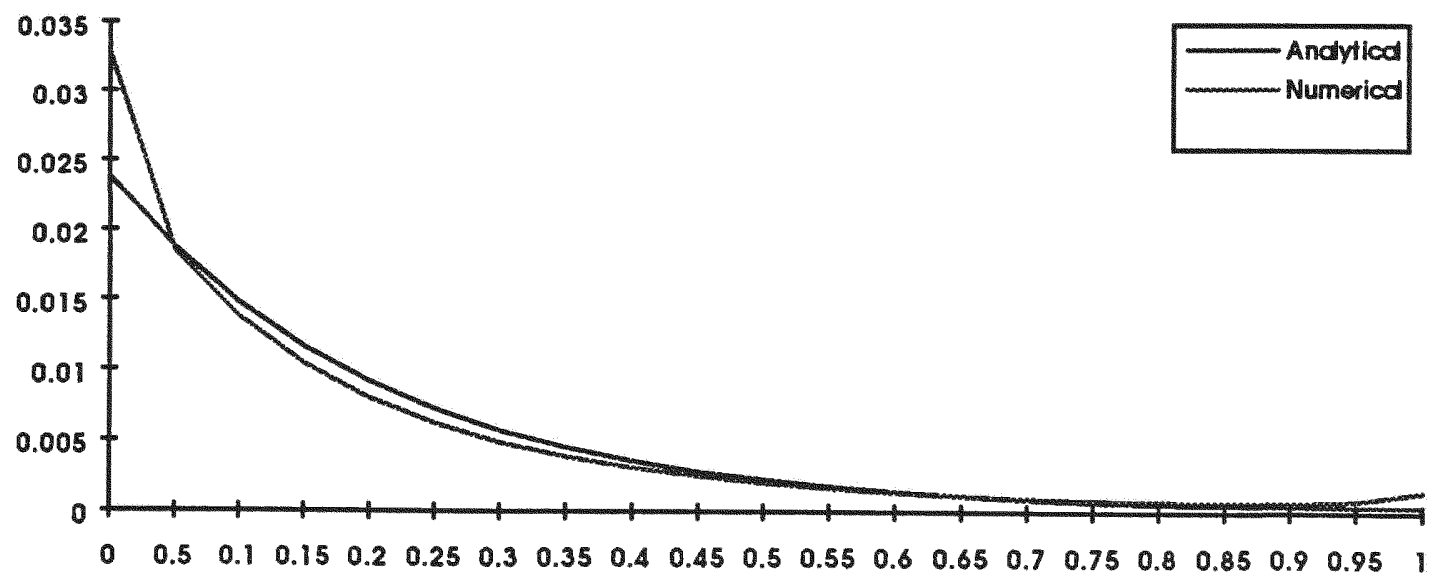

Figure 12. Graph of $\tau_{\mathrm{rz}} / \sigma_{\mathrm{o}}$ vs. $z / l$ for $\lambda=0.01$ and $\varepsilon=0.01$ 


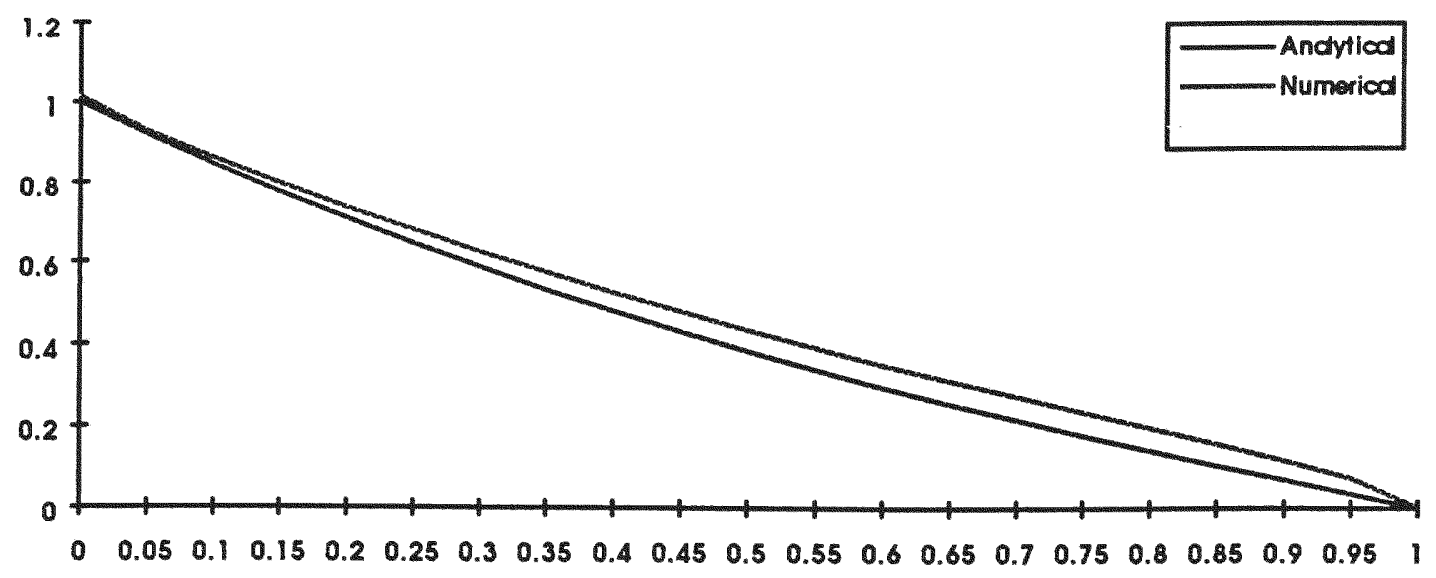

Figure 13. Graph of $\sigma_{z} / \sigma_{o}$ vs. $z / l$ for $\lambda=0.01$ and $\varepsilon=0.001$

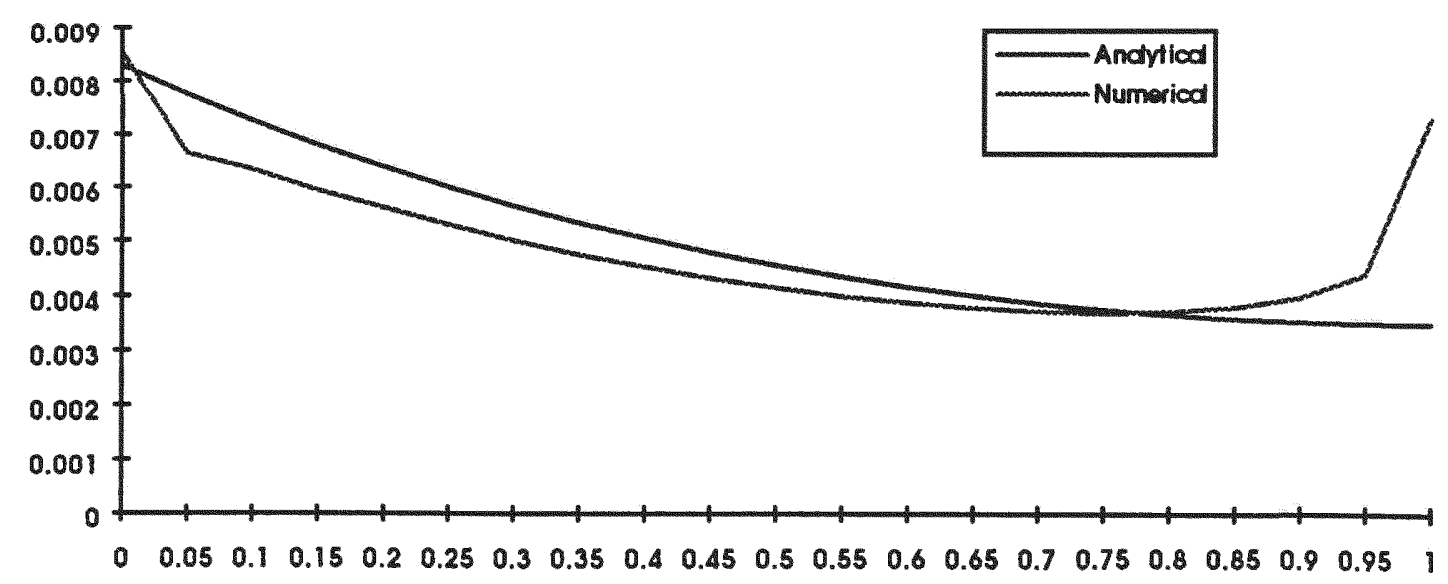

Figure 14. Graph of $\tau_{\mathrm{rz}} / \sigma_{\mathrm{o}}$ vs. $z / l$ for $\lambda=0.01$ and $\varepsilon=0.001$ 


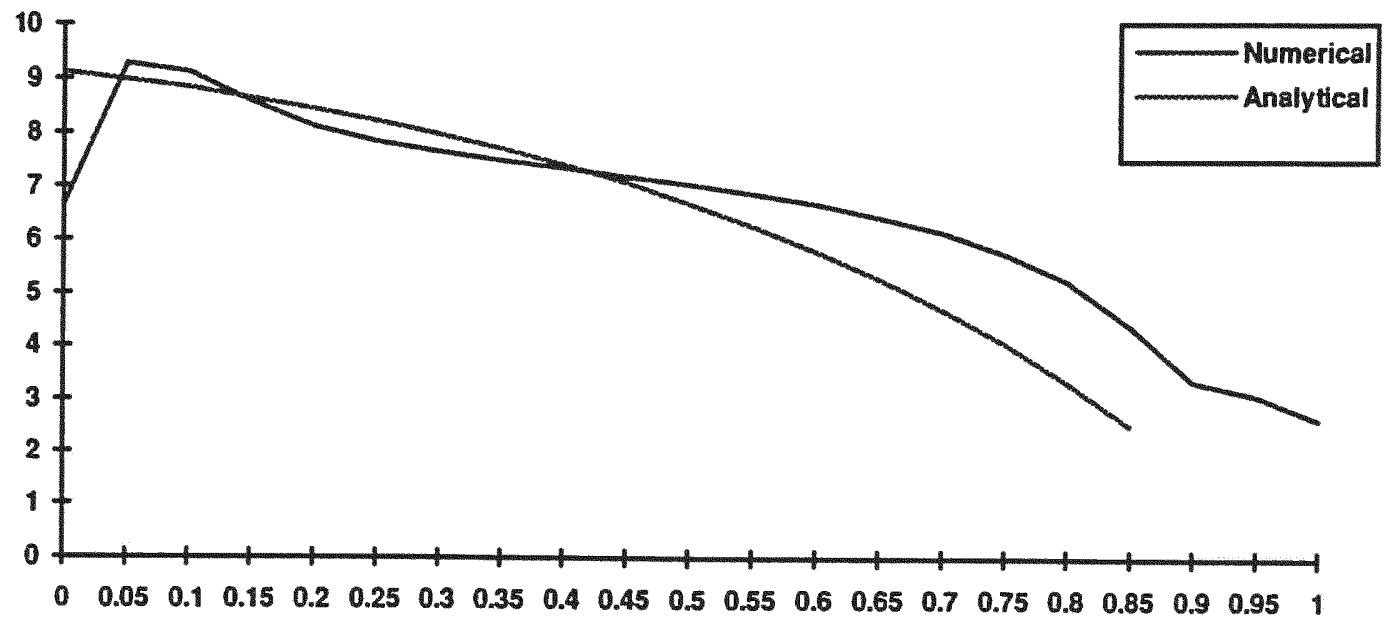

Figure 15. Graph $\sigma_{0} / \tau_{\mathrm{s}}$ vs. $l_{D} / l$ for $\lambda=0.1$ and $\varepsilon=0.1$. 


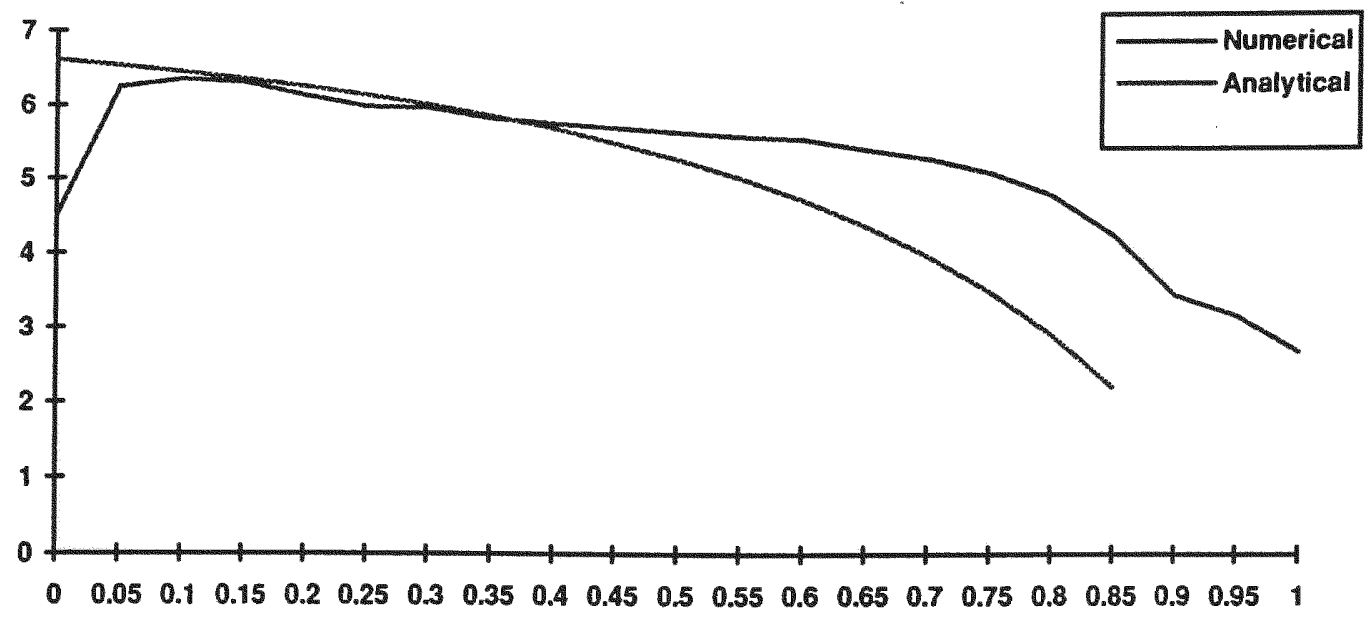

Figure 16. Graph $\sigma_{0} / \tau_{\mathrm{s}}$ vs. $l_{D} / l$ for $\lambda=0.1$ and $\varepsilon=0.2$

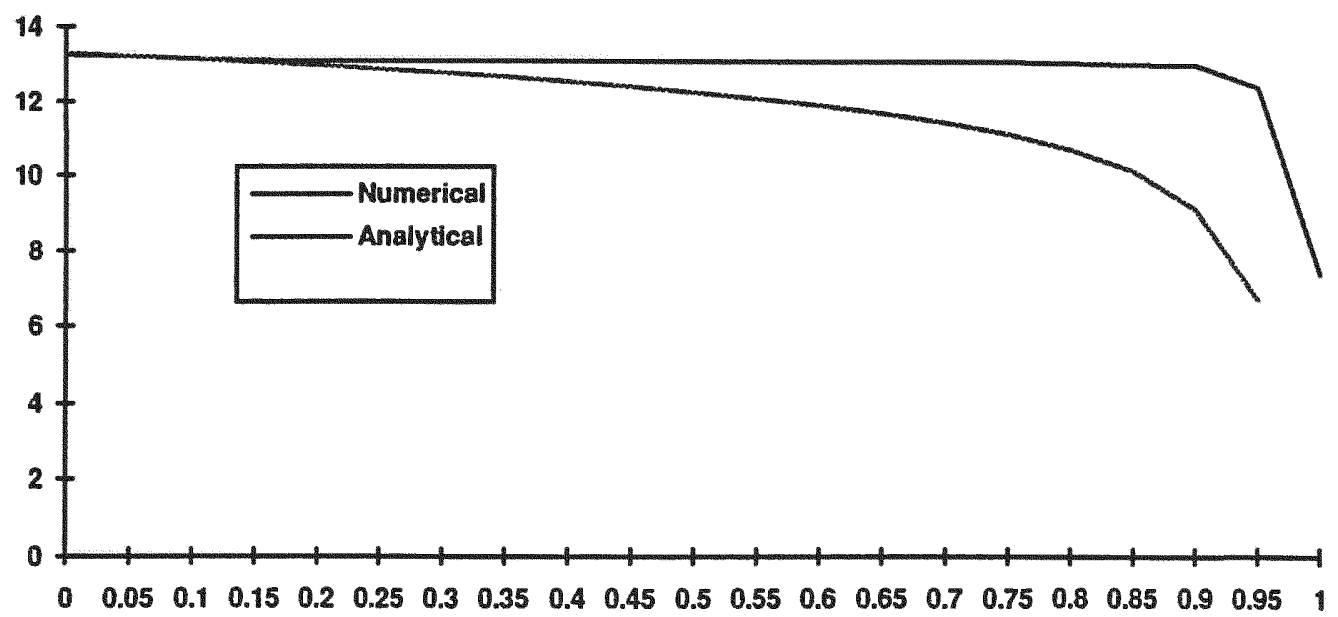

Figure 17. Graph $\sigma_{\mathrm{o}} / \tau_{\mathrm{s}}$ vs. $l_{D} / l$ for $\lambda=0.01$ and $\varepsilon=0.1$. 


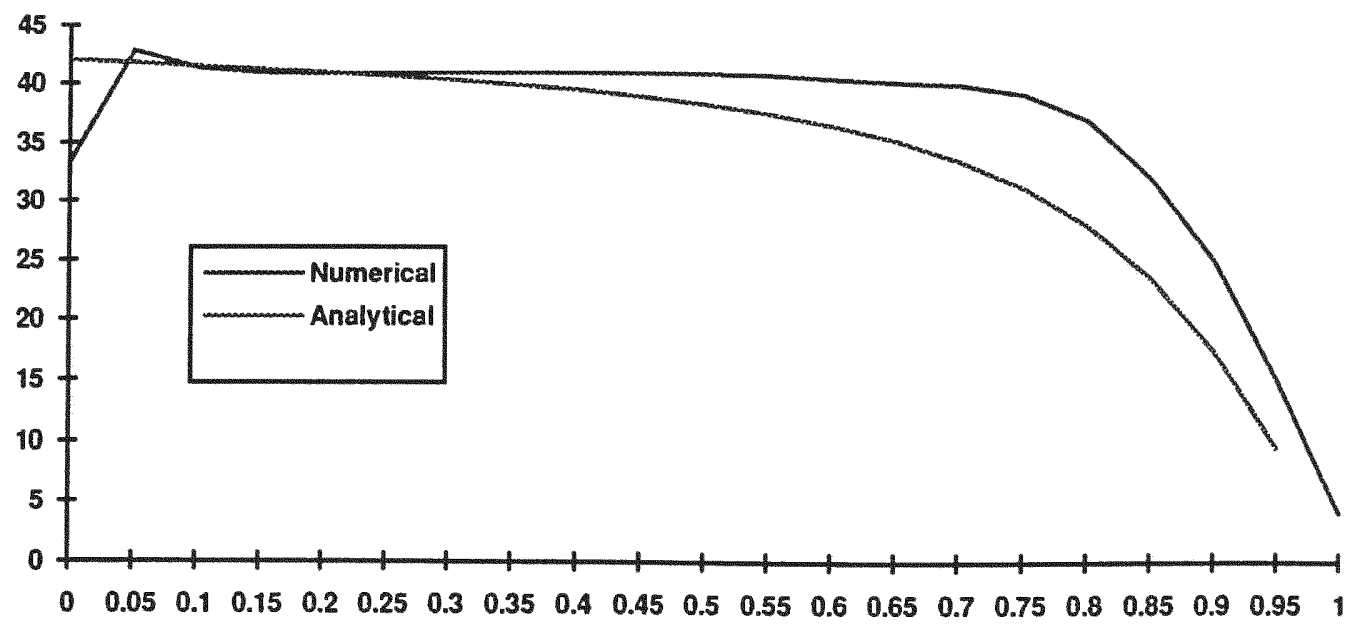

Figure 18. Graph $\sigma_{\mathrm{o}} / \tau_{\mathrm{s}}$ vs. $l_{D} / l$ for $\lambda=0.01$ and $\varepsilon=0.01$.

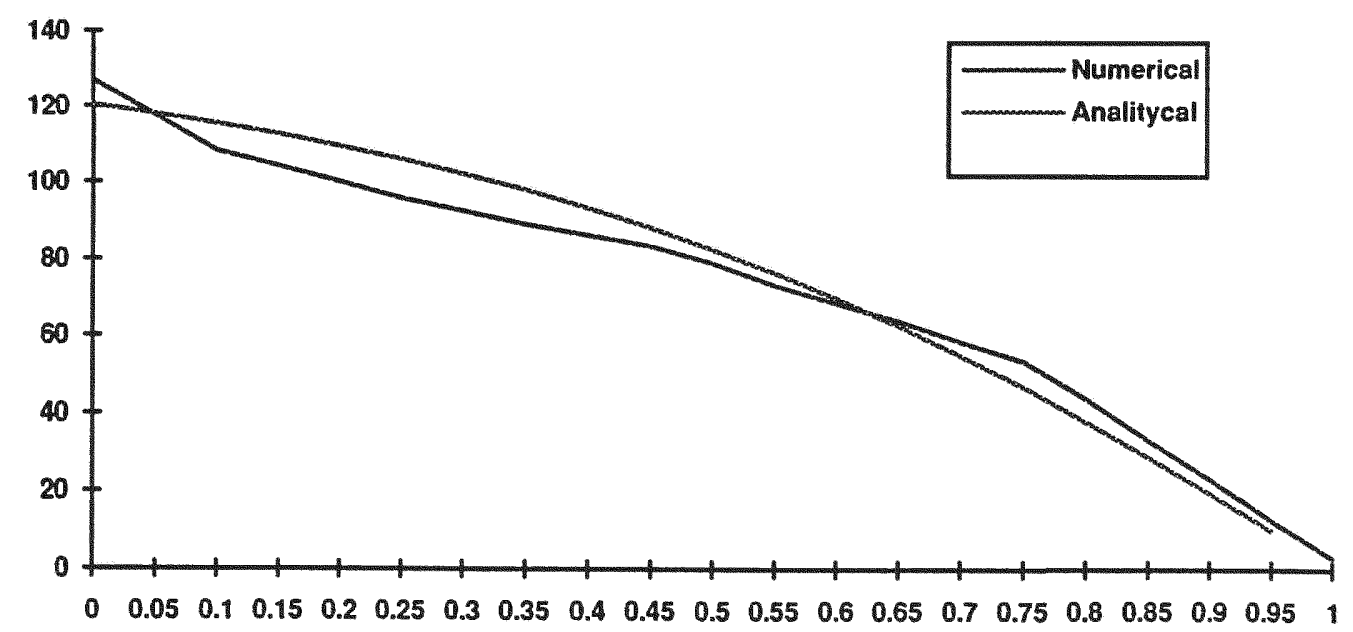

Figure 19. Graph $\sigma_{0} / \tau_{s}$ vs. $l_{D} / l$ for $\lambda=0.01$ and $\varepsilon=0.001$. 


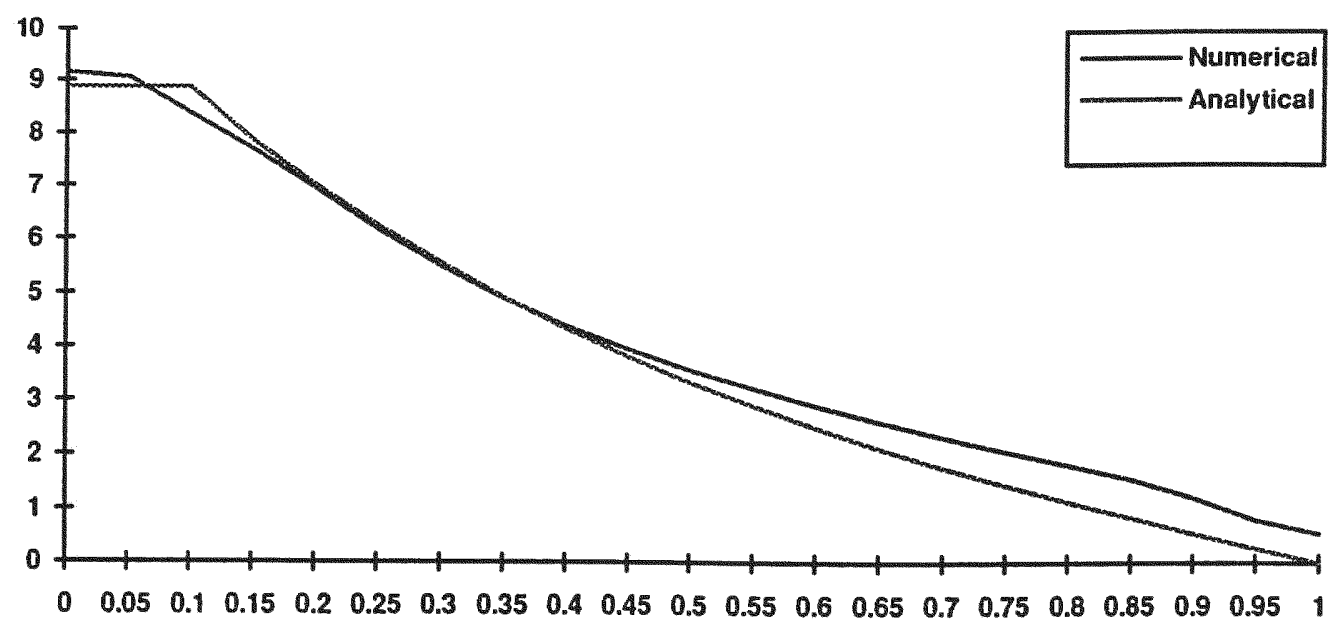

Figure 20. Graph $\sigma_{z} / \tau_{s}$ vs. $z / l$ for $\lambda=0.1, \varepsilon=0.1$ and $l_{D} / l=0.1$.

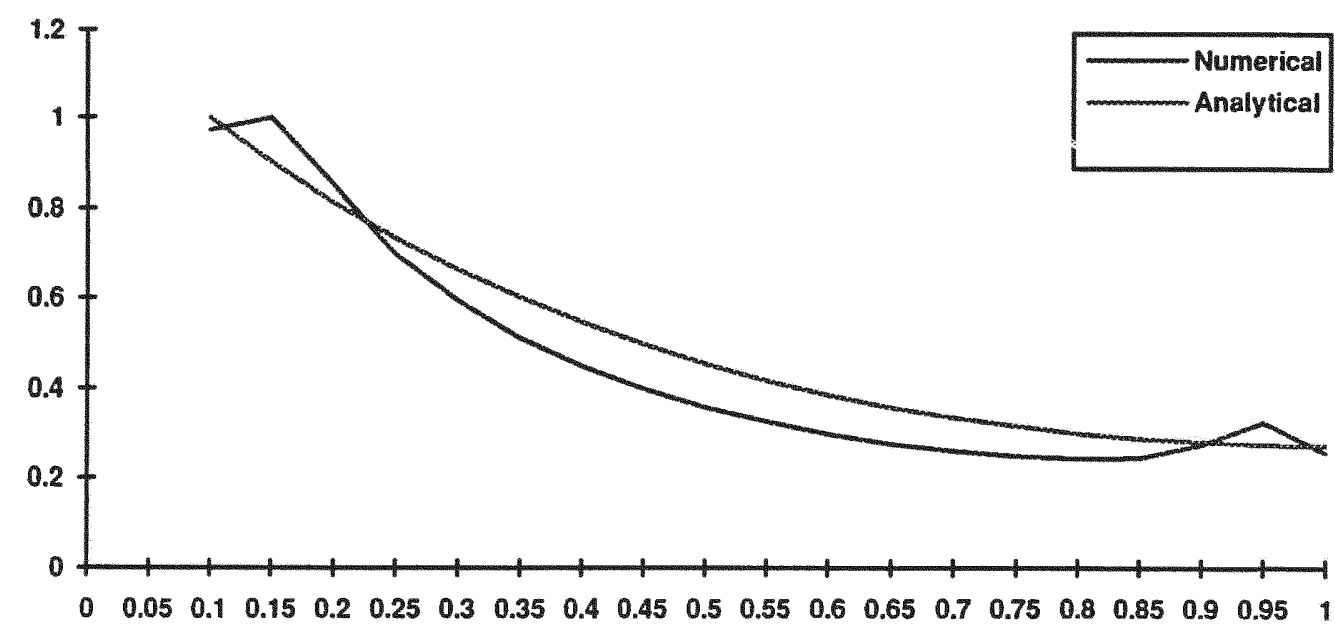

Figure 21. Graph $\tau_{\mathrm{rz}} / \tau_{\mathrm{s}}$ vs. $z / l$ for $\lambda=0.1, \varepsilon=0.1$, and $\mathrm{l}_{\mathrm{D}} / l=0.1$. 


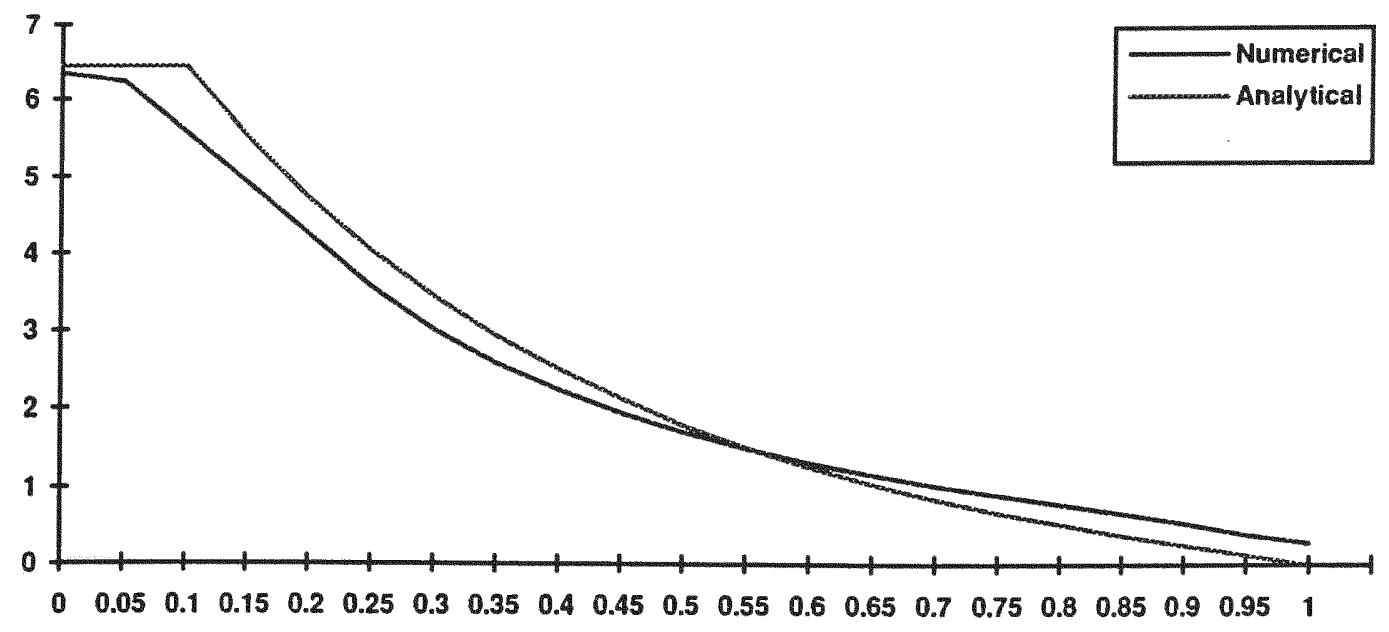

Figure 22. Graph $\sigma_{z} / \tau_{\mathrm{s}}$ vs. $z / l$ for $\lambda=0.1, \varepsilon=0.2$ and $l_{D} / l=0.1$.

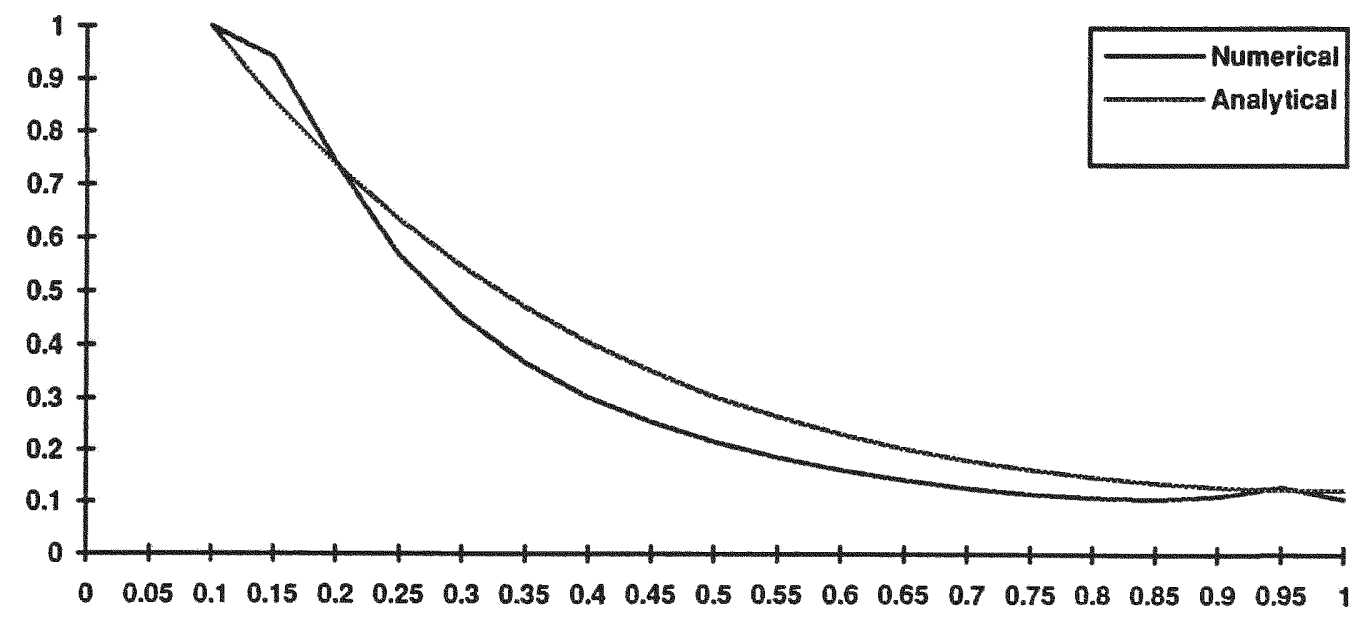

Figure 23. Graph $\tau_{\mathrm{rz}} / \tau_{\mathrm{s}}$ vs. $z / l$ for $\lambda=0.1, \varepsilon=0.2$, and $\mathrm{l}_{\mathrm{D}} / l=0.1$. 


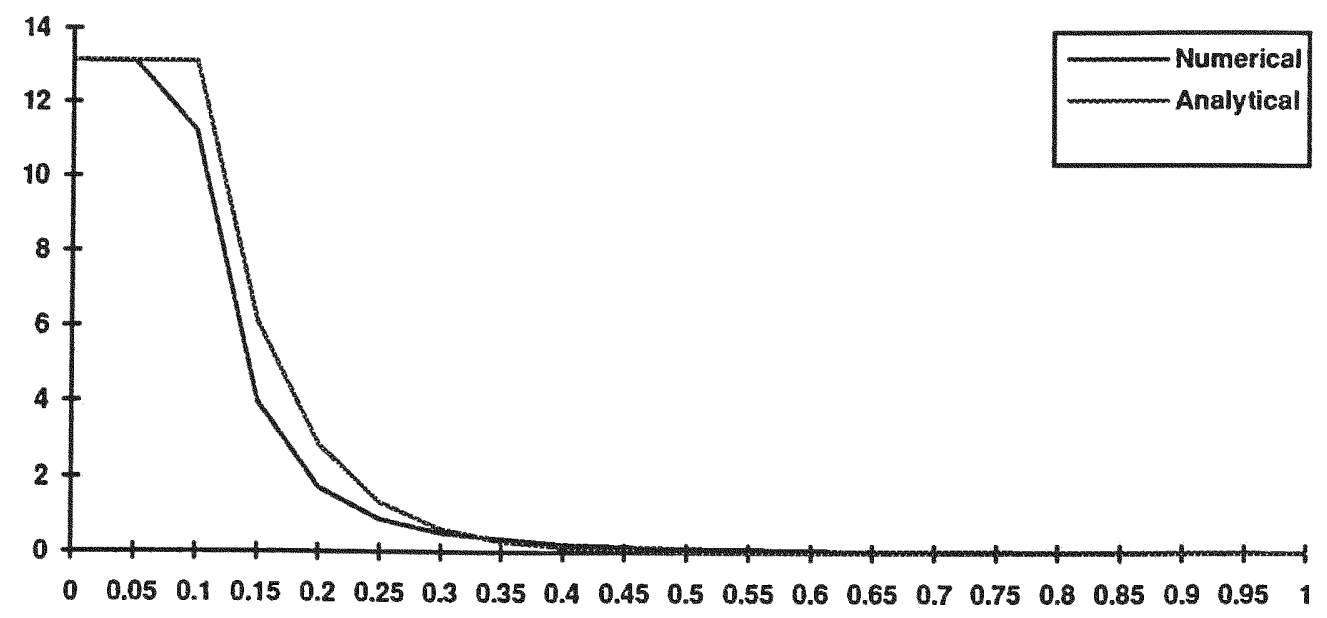

Figure 24. Graph $\sigma_{z} / \tau_{s}$ vs. $z / l$ for $\lambda=0.01, \varepsilon=0.1$ and $l_{D} / l=0.1$.

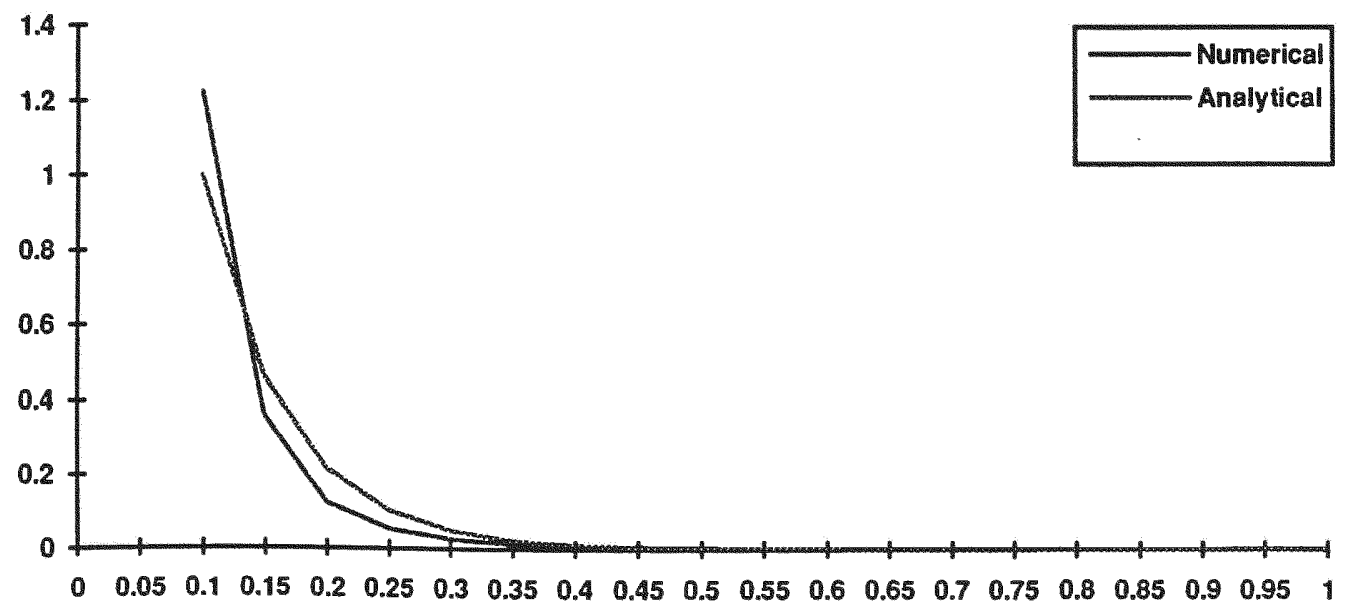

Figure 25. Graph $\tau_{\mathrm{rz}} / \tau_{\mathrm{s}}$ vs. $z / l$ for $\lambda=0.01, \varepsilon=0.1$, and $l_{\mathrm{D}} / l=0.1$. 


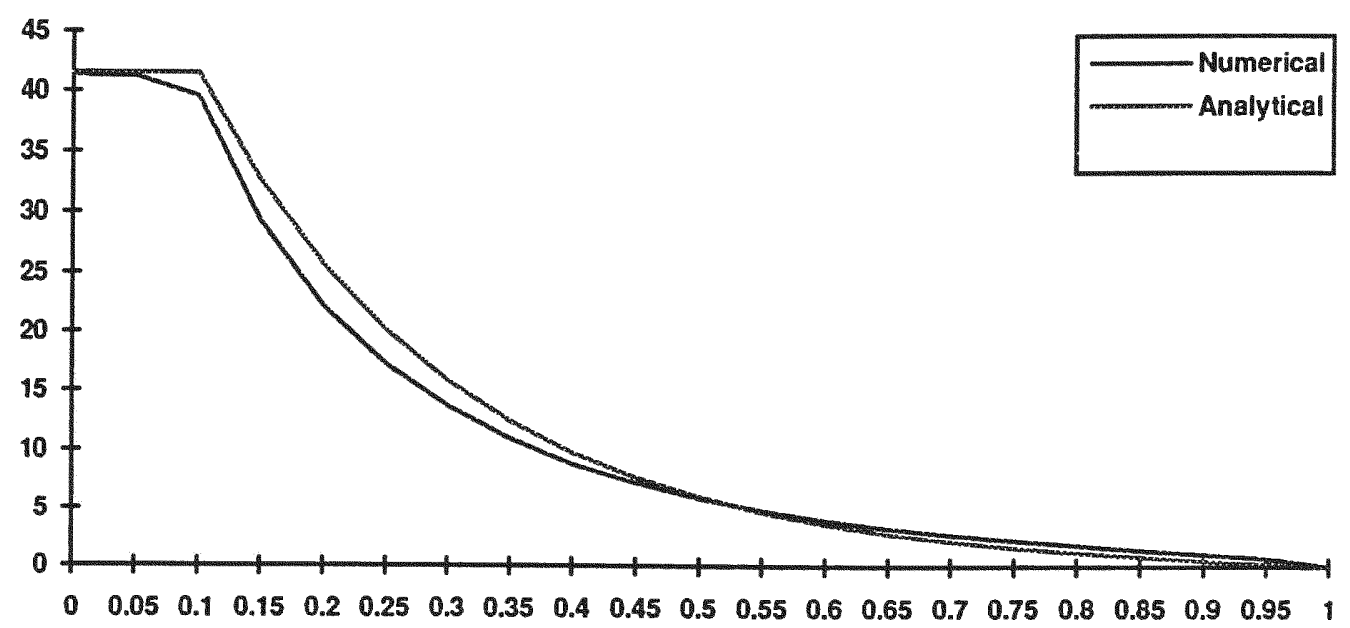

Figure 26. Graph $\sigma_{\mathrm{z}} / \tau_{\mathrm{s}}$ vs. $z / l$ for $\lambda=0.01, \varepsilon=0.01$ and $\mathrm{l}_{\mathrm{D}} / l=0.1$.

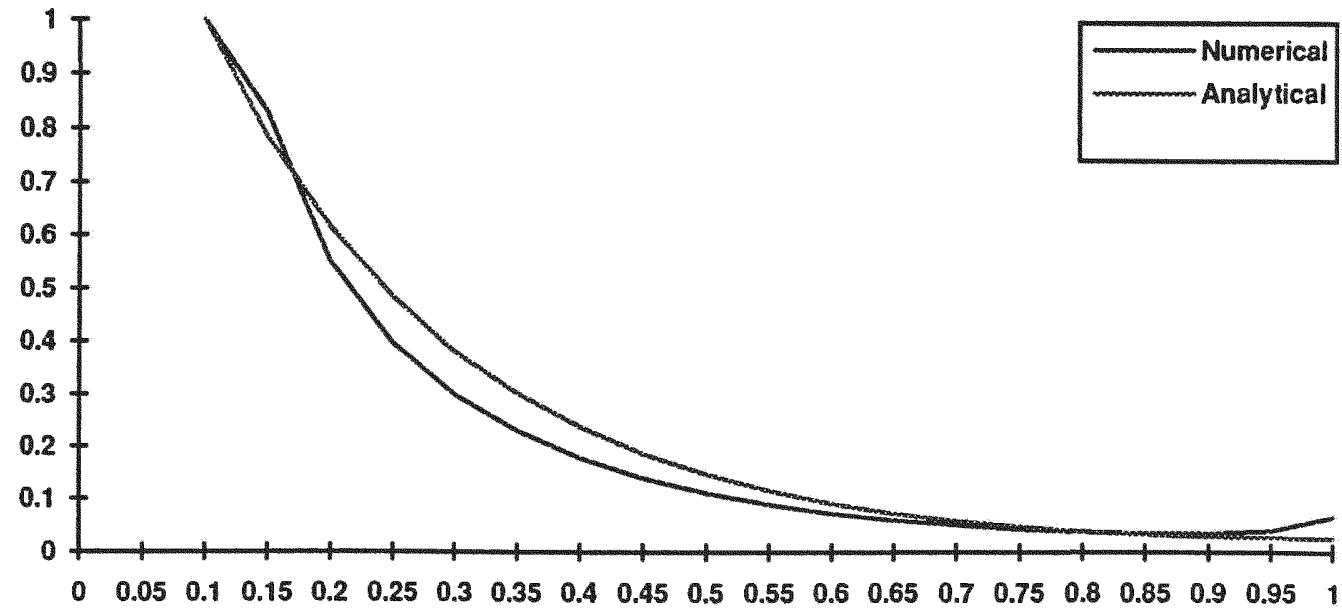

Figure 27. Graph $\tau_{\mathrm{rz}} / \tau_{\mathrm{s}}$ vs. $z / l$ for $\lambda=0.01, \varepsilon=0.01$, and $l_{\mathrm{D}} / l=0.1$. 


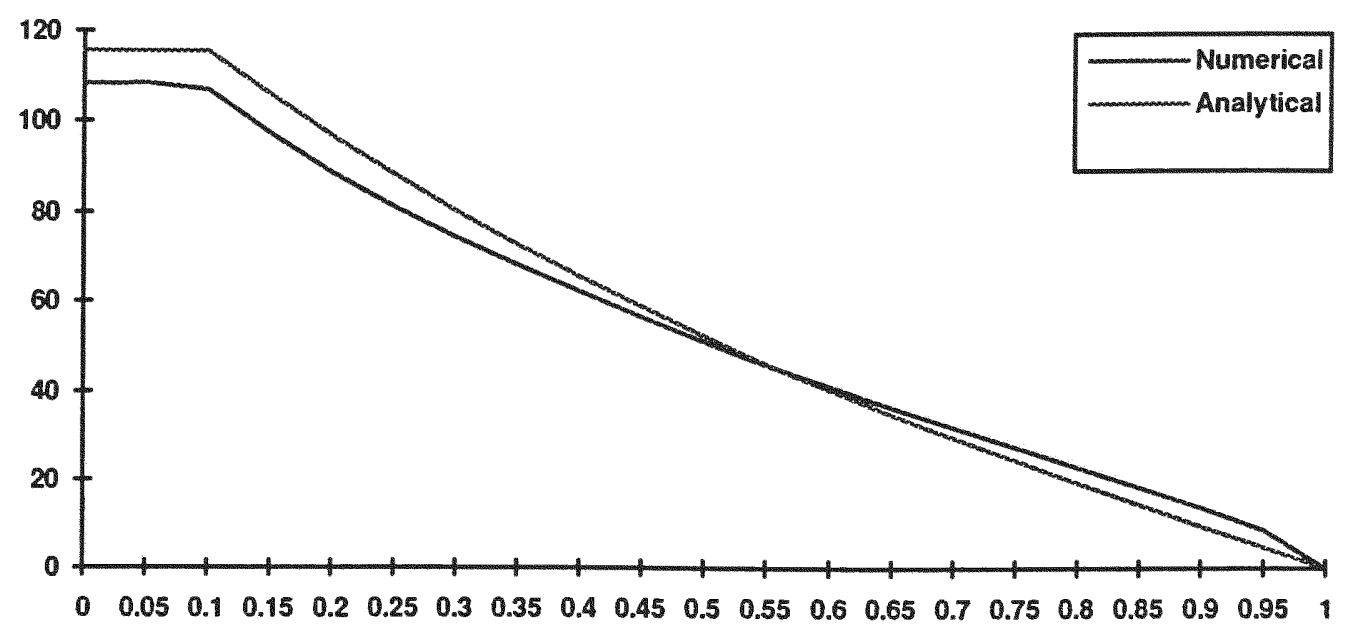

Figure 28. Graph $\sigma_{z} / \tau_{s}$ vs. $z / l$ for $\lambda=0.01, \varepsilon=0.001$ and $l_{D} / l=0.1$.

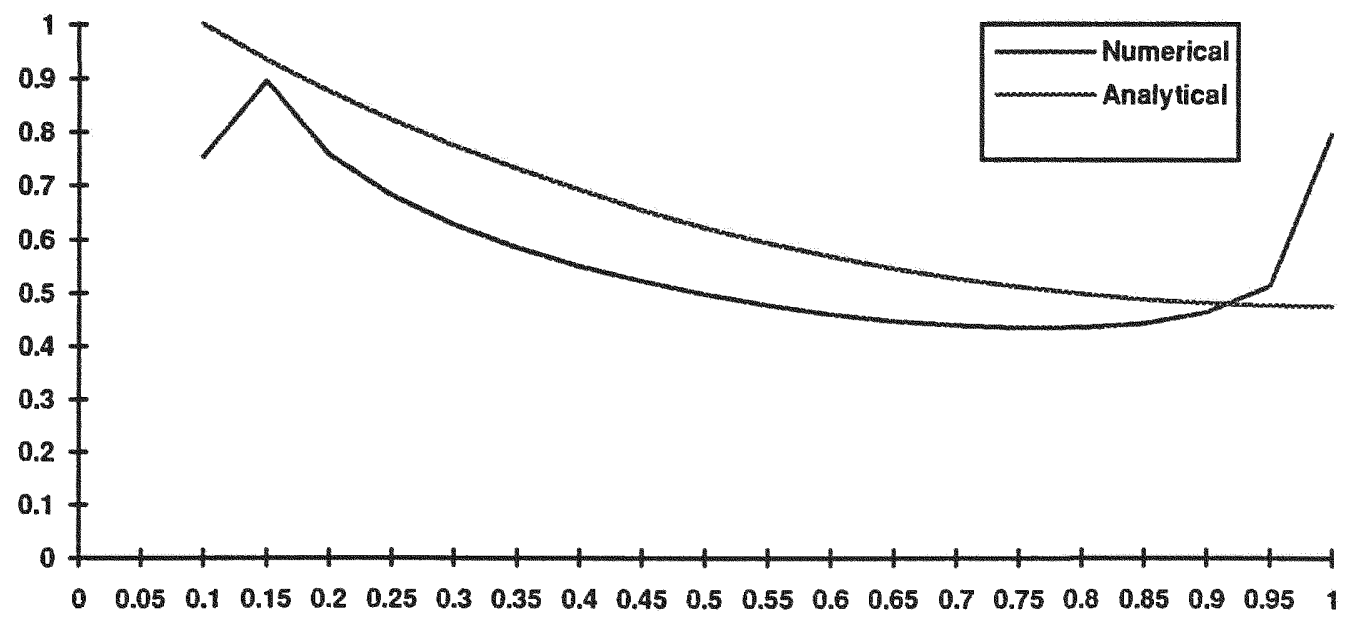

Figure 29. Graph $\tau_{\mathrm{rz}} / \tau_{\mathrm{s}}$ vs. $z / l$ for $\lambda=0.01, \varepsilon=0.001$, and $l_{\mathrm{D}} / l=0.1$. 


\section{CHAPTER 5. CONCLUSIONS AND PERSPECTIVE}

The classical problem of pullout of a long elastic fiber embedded in an elastic half-space matrix considering the cases before and while local debonding occurs has been studied numerically using the finite element method. This numerical analysis has been compared with a new theoretical approach presented in Chapter 2. The analytical solution is a completely new theoretical work different from those developed by other authors and which is based on physical principles and takes full advantage of the small relations $\lambda$ and $\varepsilon$. This approach differs from the shear lag theory developed by Cox in 1952, which is a well known theory and is the basis of many works in this area, in respect to the arrange of the fiber and the mathematical model. In the shear lag theory, the fiber under study is surrounded by other fibers which are packed in an hexagonal order at a finite distance. Besides, the transfer of load from the matrix to the fiber is defined by an assumed theoretical expression which relates the force applied at the top of the fiber with a constant and the displacements in the fiber and matrix as follows: $d F / d z=C(w-v)$, where $F$ is the force; $z$ is the axial direction; $\mathrm{C}$ is a constant that depends on the geometric arrangement of fibers, the matrix type, and the modulus of elasticity of the fiber and matrix; $w$ is the displacement in the fiber; $v$ is the displacement of the matrix away from the fiber in the $\mathrm{z}$ direction. On the contrary, in the analytical solution presented here, the fiber is embedded in a infinite half-space matrix surrounded by no other fibers and the analysis is based on the elasticity theory (Hooke's law). Analyzing both methods, it can be concluded that the shear lag theory is the solution of a particular problem while the analytical approach employed here is a solution valid for more general cases. Consequently, both solutions coincide when the 
problem suggested by Cox is analyzed using the latter approach, but the problem considered here can not be solved using the shear lag theory.

From the comparison of the results obtained here with those obtained by Muki and Sternberg (1969) and Slaughter and Sanders (1991), it can be concluded that all approaches show the same graphical behavior. However, due to the fact that $\lambda$ varies from $10^{-5}$ to $10^{-3}$ and $\varepsilon$ varies from $10^{-3}$ to $5.10^{-2}$ for practical cases of fiber-reinforced composites, the case considered by the authors mentioned above $(\lambda=0.2$ and $\varepsilon=0.5$ ) is not of practical importance for the mechanics of fiberreinforced composites. On the contrary, the cases studied here are in better agreement with the practical cases. Additionally, it is not fair to compare the results obtained by Muki et al. (1969) and Slaughter et al. (1991) with those obtained here because the analysis presented here is based on small relations $\lambda$ and $\varepsilon$ which is not taken into account in the study of the other authors. The use of the analytical solution presented here is more practical in the classical analysis of the pullout problem in the mechanics of composites for small relations $\lambda$ and $\varepsilon$ since this solution is much simpler than that complex solution developed by Slaughter and Sanders (1991).

The finite element method is a numerical method whose effectiveness in solution of engineering problems has been proved. On the other hand, the analytical method presented in Chapter 2 is based on physical principles and on intuitive ideas such as the Saint Venant's principle, boundary layer in hydrodynamics and invariant $\Gamma$-integral. From the comparison of these two different approaches, numerical and analytical, presented in Chapter 4, it can be concluded that both solutions present the same behavior. Nevertheless, based on the analysis of the 
different results studied and compared in Chapter 4 , it can be added that the correlation of both methods, analytical and numerical, depends on the dimensionless relations $\lambda=\mathrm{r}_{\mathrm{o}} / l$ and $\varepsilon=E_{m} / E_{f}$

Although the numerical technique yields estimates that are close to the exact analytical solution, there is a discrepancy, or error, due to the fact that the numerical method involved an approximation. The error can be computed exactly if there is an exact analytical solution available. For the problem studied here there is no exact analytical solution but an approximate analytical solution. Therefore, the errors associated with the numerical method can not be computed exactly. However, it is important to notice that different facts such as the truncation error, the presence of different materials in the selected element set, the poor aspect ratio of some elements after element meshing (mainly when $\mathrm{r}_{\mathrm{o}} / l$ $=0.01$ ), and the modeling procedure of the debonding problem in the finite element analysis using ANSYS should be considered to estimate the real error of the finite element method.

It is strongly recommended that the results obtained in this investigation be compared with physical experiments results in order to establish the real error of the approaches studied. This comparison was not made in this work because no such data of the pullout problem are available.

This work represents a tool to understand the mechanism of load transfer from the matrix to the fiber through the interface when the fiber is subjected to a pullout force. From the load transfer mechanics viewpoint, these analytical and numerical solutions give the distribution of stresses along the fiber. They also 
show the stress state at the interface which is of special interest because the fibermatrix interface, which for all practical purposes consists of the bond between the fiber and matrix, gives fiber composites their structural integrity.

From the two problems considered here, the elastic pullout problem and the debonding problem by pullout, it can be concluded that in the elastic pullout problem the structural integrity of the composite remains; as a result, the composite works as a new elastic material. For this case, the bed coefficient $\left(\mathrm{B}=\mathrm{F} / W_{\max }\right)$ can be defined as an elastic constant for any pair of materials which constitute the fiber composite. This coefficient is valid until the maximum stress developed in the fiber reaches certain critical value producing plastic deformation or forcing the bond to break at the interface.

In contrast, when certain maximum load is applied at the top of the fiber, the limiting shear stress is reached at the interface forcing the bond to break. Then, the length of debonding starts growing but this growth is unstable, which means that the load decreases while the length of debonding grows. So that, the structural integrity disappears and the composite fails. From the analysis of Figs. $5,7,9,11$, and 13 no stable growth of $l_{D}$ is expected after the top of the interface is reached, although the numerical results for $\lambda=0.1, \varepsilon=0.1$ and 0.2 , as well as $\lambda$ $=0.01, \varepsilon=0.01$ show a stable growth until approximately $5 \%$ of the total length. This effect should be due to the numerical model instead of the physical phenomenon.

The great advantage of the results presented is that they are expressed in dimensionless form. It is important to observe that these results are function 
only of the following dimensionless parameters: $\lambda$, $\varepsilon$, and $\nu_{\mathrm{m}}$ (Poisson's ratio of the matrix). From this, it can be concluded that the theoretical solution of the pullout problem developed in Chapter 2 is independent of the Poisson's ratio of the fiber $\left(v_{f}\right)$. This dimensionless analysis is not only important because any system of units can be used but also because it helps to find in a relative simple manner the best pair of material combination $\left(\lambda, \varepsilon\right.$, and $\left.\nu_{\mathrm{m}}\right)$ in the design process knowing the working stress $\left(\sigma_{\mathrm{o}}\right)$.

Supplementary work can be undertaken related to the numerical analysis of this problem by improving the debonding problem model. For this case a different set of matrix-elements can be defined along the length of debonding on the interface. In addition, a specific analysis of the singularity at the debond zone tip can be made by means of a more advanced finite element method using, for instance, a similar model to that used for crack tip analysis.

This work can be employed as a base for future investigations in this area wherein other factors are considered such as initial or residual stresses, inelastic behavior of a fiber and/or a matrix, interaction of many fibers, temperature and technological effects and so on. Additionally, the theoretical ideas presented in Chapter 2 lead to a wide range of problems that can be considered where those ideas may be applied. These problems include magnetic and electromagnetic effects of the fiber composite among others. 


\section{REFERENCES}

Bao, G., and Hui, C.Y., 1990, "Effects of interface debonding on the toughness of ductile-particle reinforced ceramics", International Journal of Solids and Structures, 26, (5-6), 631-642.

Becher, P.F., Hsueh, C. H., Angelini, P., and Tiegs, T. N., 1988, "Toughening behavior in whisker-reinforced ceramic matrix composites", J. Am. Ceram. Soc., 71 (12), 1050-1061.

Bright, J.D., Shetty, D.K., Griffin, C.W., and Limays, S.Y., 1989, "Interfacial bonding and friction in SiC-reinforced ceramic and glass matrix composites", J. Am. Ceram. Soc., 72 (10), 1891-1898.

Broutman, L.J., and Krock, R.H., 1974, " Composite Materials. Interfaces in polymer matrix composites", New York, Academic Press, 6, 294.

Brun, M.K. and Singh, R.N., 1988, "Effect of thermal expansion mismatch and fiber coating on the fiber/matrix interfacial shear stress in ceramic matrix composites", Adv. Ceram. Mater., 3(5), 506-509.

Budiansky, B. and Amazigo, J.C., 1989, "Toughening by aligned, frictional constrained fibers", J. Mech. Phys. Solids, 37, 93-109.

Budiansky, B., Hutchinson, J.W., and Evans, A.G., 1986, "Matrix fracture in fiber-reinforced ceramics", J. Mech. Phys. Solids, 34 (2), 167-189.

Buxianidze, A. A. and Cherepanov, G. P., 1986, "A problem of cooling an isolated heat-generating bar by fluid flow", Boundary Value Problems and Their Applications, Cheboxary, Chuvash University Press., 84-97.

Buxianidze, A. A. and Cherepanov, G. P., 1986, "On the heat exchange between a rod and a medium for small Peclet numbers", Mechanics of Continuum, Tbilisi, Georgia Polytechnic Institute Press., 172-181.

Buxianidze, A. A. and Cherepanov, G. P., 1986, "Thermal conductivity of a wall having thin cylindrical inclusions", Soviet Materials Science, 4, 637-641.

Chawla, K.K., 1987, "Composite materials. Science and engineering", New York, Springer-Verlag, 292. 
Chen, Y.C., and Hui, C.Y., 1990 "Load transfer in a composite containing a broken fiber with imperfect bonding", Mechanics of Materials, 10, (3), 161-172.

Cherepanov G.P., 1981 "Invariant Г-integrals", Eng. Fracture Mechanics, $14,(1), 39-58$.

Cherepanov G.P., 1983, "Fracture Mechanics of Composite Materials", Moscow, Nauka Publishers, 308 , (In Russian).

Cherepanov, G. P., 1985, "On the Opening Up of Oil and Gas Boreholes", Doklady of the USSR Academy of Sciences, 284, (4), 1845-49.

Cherepanov, A. G. and Cherepanov, G. P., 1985, "On the stresses in a fiber inclusion in a visco-elastic matrix", Soviet Appl. Mechanics, 22, (2), 321-330.

Cherepanov, G. P. and Esparragoza, I. E., 1992, "Elastic Pullout Problem of a Fiber Embedded in a Matrix", Submitted to Journal of Applied Mechanics.

Cherepanov, G. P. and Esparragoza, I.E., 1993, "The Debonding Problem of a Fiber Embedded in a Matrix by Pullout", Submitted to J. of Com. Materials.

Cherepanov, G.P., 1993, "Some novel approaches in mechanics of composites", Invited paper for the Symposium on Composite Materials and Structures at the 1993 ASME Winter Annual Meeting, New Orleans.

Cox, H.L., 1952, British Journal of Applied Physics, 3, 72-79.

Delale, F., and Xu, X.L., 1990 "Effect of fiber interaction on matrix and interfacial microcracking in ceramic matrix composites", Winter annual meeting of the American Society of Mechanical Engineers, Aerospace Division (Publication) AD v19. Publ. by Fract. Mech., 31, (1), 145-155.

Dollar, A. and Steif, P.S., 1988, "Load transfer in composites with a Coulomb friction interface", Int. J. Solids Structures, 24, (8), 789-803.

Eshelby, J.D., 1982, "The stresses on and in a thin inextensible fiber in a stretched elastic medium", Eng. Fract. Mech., 16, (3), 453.

Evans, A. G., 1991, "The mechanical properties of reinforced ceramic, metal and intermetalic matrix composites", J. Mat. Sci. and Eng., A 143, 63-76.

Friedrich, K., 1989, "Application of fracture mechanics to composite materials", Elsevier, New York. 
Gao, Y.C., Mai, Y.W. and Cotterell, B., 1988, "Fracture of fiber-reinforced materials", J. App. Math. Physics, (ZAMP) 39, 550-572.

He, M., and Hutchinson, J.W., 1989, "Crash deflection at and interface between dissimilar elastic materials"' Int. J. Solids Struct., 25, 1053-1057.

Hsueh, C.H., 1988, "Elastic load transfer form partially embedded axially loaded fiber to matrix", J. Mat. Sci., Lett., 7, (5), 497-500.

Hsueh, C.H., 1990, "Interfacial debonding and fiber pull-out stresses of fiber reinforced composites", J. Mat. Sci. and Eng., A 123, 1-11.

Hsueh, C.H., 1990, "Interfacial frictional analysis for fiber-reinforced composites during fiber push-down", J. Mat. Sci., in the press.

Hsueh, C.H., 1990, "Interfacial debonding and fiber pull-out stresses of fiber reinforced composites. II: non-constant interfacial bond strength", J. Mat. Sci and Eng., A 125, 67-73.

Hsueh, C.H., 1990, "Interfacial debonding and fiber pull-out stresses of fiber reinforced composites. III: with residual radial and axial stresses", J. Mat. Sci and Eng., A145, 135-142.

Hsueh, C.H., 1990, "Interfacial debonding and fiber pull-out stresses of fiber reinforced composites. IV: sliding due to residual stresses", J. Mat. Sci. and Eng., A145, 143-150.

Hutchinson, J.W., 1990 "Mixed mode fracture mechanics of interfaces, in: Metal-ceramic interfaces, Eds. M. Ruhele, A.G. Evans, M.F. Ashby, and J.P. Hirth, Pergamon Press, New York, 295-306.

Hutchinson, J. W., and Suo, Z., 1992, "Mixed mode cracking in layered materials", Advances in Applied Mechanics, 29, 63-191.

Hutchinson, J. W. and Jensen, H. M., 1990, "Models of fiber debonding and pull-out in brittle composites with friction", Mech. Mater., 9, 139-163.

Jones, R.M., 1975, "Mechanics of composite materials", Washington, Scripta Book Co., 355.

Kelly, A., and Davies, G. I., 1965, "The principles of the fiber reinforcement of metals", Metallurgical Reviews, 10, (37), 1-78. 
Kerans, R.J. and Parthasarathy, T.A., 1991, "Theoretical analysis of the fiber pullout and pushout tests", J. Am. Ceram. Soc., 74, (7), 1585-1596.

Kim, J.K., Baillie, C., and Mai, Y.W., 1991, "Instability of interfacial debonding during fiber pull-out", Scripta Metallurgical et Materialia, 25, (2), 315320 .

Kishi, T. , Enoki, M., and Tsuda, H., 1992, "Interface and strength in ceramic matrix composites", Proceedings of the Joint Japan-USA seminar on Advanced Materials for Severe Service Applications.

Landau, L. D. and Lifshitz, E.M., 1990, "Electrodynamics of Continuous Media", Addison-Wesley, Reading, Massachusetts.

Lawrence, P., 1972, "Some theoretical considerations of fiber pull-out from an elastic matrix", J. Mat. Sci, 1, (1), 1-6.

Li, Z., Mobasher, B. and Shah, S.P., 1991, "Characterization of interfacial properties in fiber-reinforced cementitious composites", J. Am. Ceram. Soc., 74, (9), 2156-2164.

Liang, C. and Hutchinson, J.W., 1992, "Mechanics of the fiber pushout test", Mech. of Mat., 14, 207-221.

Majumdar, S., Singh, D., and Singh, J.P., 1993, "Analysis of pushout test on an $\mathrm{SiC}$-fiber-reinforced reaction-bonded $\mathrm{Si}_{3} \mathrm{~N}_{4}$ composite", J. of Composite Eng., 3, 287-312.

Mandell, J.F., Hong, K.C.C. and Grande, D.H., 1987, "Interfacial shear strength and sliding strength in metal and glass-ceramic matrix composites", Ceram. Eng. Sci. Proc., 8, (7-8), 937-940.

Marshall, D.B. and Oliver, W.C., 1987, "Measurement of interfacial mechanical properties in fiber-reinforced ceramic composites", J. Am. Ceram. Soc., $70,(8), 542-548$.

McCartney, L. N., 1989, "New theoretical model of stress transfer between fiber and matrix in a uniaxially fiber-reinforced composite", Proc. R. Soc. (London), A 425, 1868, 215-244.

Morley, J.G., 1987, "High-Performance fibre composites", Orlando, Academic Press, 238. 
Morscher, G., Pirouz, P. and Heuer, A.H., 1990, "Temperaturedependence of interfacial shear strength in $\mathrm{SiC}$ fiber-reinforced reaction-bonded $\mathrm{Si}_{3} \mathrm{~N}_{4}$ ", J. Am. Soc., 73, (3), 713-720.

Muki, R. and Sternberg, E., 1969, "On the diffusion of an axial load from an infinite cylindrical bar embedded in an elastic medium", J. Solids Structures, $5,587-605$

Muki, R. and Sternberg, E., 1970, "Elastostatic load-transfer to a half-space from a partially embedded axially loaded rod", J. Solids Structures, 6, 69-90.

Mura, T., Brittain, J. O., and Faber, K., 1990, "Micro mechanics of interfaces in high temperature ceramics", Annual Report No. 1, FY1989-FY1990, $58,28$.

Murri, G. B., and Guynn, E.G., 1988, "Analysis of delamination growth from matrix cracks in laminates subjected to bending loads"' Composite Materials: Testing and Design, eighth Conference, Charleston, SC, USA, 29 Apr.1 May. 1986, Report No. STP 972, Publ: Philadelphia, Penn., 332-339.

Nair, S.V., 1990 "Crack-wake debonding and toughness in fiber-or whisker-reinforced brittle-matrix composites", J. Ame. Ceram. Soc., 73, (10), 2839-2847.

Nikishkov, G. P. and Cherepanov, G. P., 1984, "Extension of an elastic space having an isolated rigid round inclusion", Appl, Math. Mech. (PMM), 48, (3), 581-594.

Ortiz, M., and Blume, J.A., 1990 "Effects of decohesion and sliding on bimaterial crack-tip fields", International Journal of Fracture, 42, (2), 117-128, Feb.

Pak, R. Y. S., 1989, "On the flexure of a partially embedded fiber under lateral loads", J. Appl. Mech., 56, 263-269.

Parthasarathy, T. A., Jero, P. D., and Kerans, R. J., 1991, "Extraction of interface properties from a fiber pull-out test", Scripta Metallurgica et Materialia, $25,(11), 2457-2462$.

Phillips, D.C., 1974, "Interfacial bonding and the toughness of carbon fiber reinforced glass and glass ceramics", J. Mat. Sci., 9, 1874-1885. 
Piggot, M.R., 1980, "Load bearing fibre composites", London, Pergamon Press, 277.

Rice, J. R., Suo, Z, and Wang, J. S., 1990, "Mechanics and thermodynamics of brittle interfacial failure in bimaterial systems", Metal-Ceramic Interfaces (Eds. M. Rühle, A. G. Evans, M. F. Ashby and J. P. Hirth), Acta-Scripta Metallurgica Proceedings Series, Vol. 4, Pergamon Press, 269-294.

Slaughter, W.S., 1993, "A self-consistent model for multi-fiber crack bridging", Int. J. Solid Struct., 30, (3), 385-398.

Slaughter, W. S., and Sanders, J. L., 1991, "A model for load-transfer from an embedded fiber to an elastic matrix", J. Solids Structures, 28, 1041-52.

Stang, H., Li, Z., and Shah, S. P., 1990 "Pullout problem, stress versus fracture mechanical approach", J. Eng. Mech., 116, (10), 2136-2150.

Sternberg, E., 1970, "Load transfer and load-diffusion in elastostatics", Proc. Sixth U.S. National Congress of Applied Mechanics, 34-61.

Sutcu, M., and Hilling, W. B., 1990, "Effect of fiber-matrix debond energy on the matrix cracking strength and the debond shear strength", Acta Metallurgica et Materialia, 38, (12), 2653-2662.

Takaku, A., and Arridge, R. G. C., 1973, "The effect of interfacial radial and shear stress on fiber pull-out in composite materials", J. Phys., D, 6, 20382047.

Thouless, M.D., and Evans, A. G., 1988, "Effects of pull-out on the mechanical properties of ceramic matrix composites", Acta Metallurgica et Materialia, 36, 517-522.

Thouless, M.D., Sbaizero, O., Sigl, L.S., and Evans, A. G., 1989, "Effect of interface mechanical properties on pull-out in SiC-fiber-reinforced LAS glass ceramics", J.Am. Ceram. Soc., 72, 525-532.

Weeton, J.W., Peters, D.M., and Thomas, K.L., 1987, "Engineers' guide to composite materials", Ohio, American Society for Metals, 14-1.

Wells, J.K., and Beaumont, P. W. R., 1985, "Debonding and pull-out processes in fibrous composites", J. Mat. Sci., 20, (4), 1275-1284. 
Zimmerman, K.A., Langford, S. C., and Dickinson, J.T., 1991, "Electrical transients during interfacial debonding and pull-out of a metal rod from an epoxy matrix", Washington State University, J. Appl. Phy., 70, (9), 4808-4815. 


\section{APPENDICES}

\section{A. MODELING PROCEDURE OF THE PROBLEM}

Here, all the information necessary to complete the first step of the modeling procedure is given in extensive form in order to make clear this basic step.

Due to the fact that the full ANSYS program is able to perform different types of analyses, a specific analysis to be performed must be indicated. Thus, the correct equation can be solved and the program can perform various automatic checks of the data that has been entered. For the problem under consideration the static analysis is the suitable option. This is specified in the following form:

$\mathrm{KAN}, 0$

where KAN represents the analysis choice, and 0 represents the static analysis.

After that, the element type is chosen from the ANSYS element library. Because of the specialization of the elements in some way, the element must be selected so that it corresponds to the type of analysis which will be performed. ET command is the instruction used to select elements from the library. The element is selected for this work using the following command:

$$
\mathrm{ET}, 1,82,1
$$

which means that this is an element type 1 (local name), with stiffness 82 (twodimensional multinode isoparametric solid element), and axisymmetric property.

The next stage is to define the material properties. The command EX is used to determine the Young's modulus (considering the material isotropic, only the $x$ 
direction value needs to be defined), and the command NUXY is the label used for Poisson's ratio.

The material properties for material 1 (fiber) are specified as follow:

$$
\begin{aligned}
& \text { EX, 1, N1 } \\
& \text { NUXY, 1, } 0.3
\end{aligned}
$$

The material properties for material 2 (matrix) are specified as follow:

$$
\begin{aligned}
& \text { EX, 2, N2 } \\
& \text { NUXY, 2, } 0.3
\end{aligned}
$$

Here, N1 and N2 mean the different values of Young's modulus that are considered. The terms N1 and N2 are used because this work includes the analysis of different relations Young's modulus of matrix $\left(\mathrm{EX}_{\mathrm{m}}\right) /$ Young's modulus of fiber $\left(E X_{f}\right)$. 


\section{B. RESULTS OF THE ELASTIC PULLOUT PROBLEM}

All the results of the elastic pullout problem are presented here. The results of the distribution of the normal stresses are given in Tables 1 to 5 . The results of the distribution of shear stresses are compiled in Tables 6 to 10. The results of the maximum displacement come in Table 11. 
Table 1. Results of the distribution of normalized $\sigma_{z}$ along the total embedded length of the fiber for the elastic pullout problem in the case of $\lambda=0.1$ and $\varepsilon=0.1$.

\begin{tabular}{lcc}
\hline $\mathrm{z} / l$ & $\sigma_{\mathrm{Z}} / \sigma_{\mathrm{o}}$ (Numerical) & $\sigma_{\mathrm{Z}} / \sigma_{\mathrm{o}}$ (Analytical) \\
0.00 & 1.012 & 1.000 \\
0.05 & 0.957 & 0.896 \\
0.10 & 0.824 & 0.802 \\
0.15 & 0.711 & 0.717 \\
0.20 & 0.631 & 0.640 \\
0.25 & 0.567 & 0.571 \\
0.30 & 0.512 & 0.508 \\
0.35 & 0.464 & 0.451 \\
0.40 & 0.420 & 0.399 \\
0.45 & 0.381 & 0.351 \\
0.50 & 0.346 & 0.308 \\
0.55 & 0.314 & 0.268 \\
0.60 & 0.284 & 0.231 \\
0.65 & 0.256 & 0.197 \\
0.70 & 0.230 & 0.164 \\
0.75 & 0.204 & 0.134 \\
0.80 & 0.179 & 0.105 \\
0.85 & 0.153 & 0.078 \\
0.90 & 0.120 & 0.051 \\
0.95 & 0.079 & \\
1.00 & 0.054 & \\
\hline & & \\
0.000 \\
0
\end{tabular}


Table 2. Results of the distribution of normalized $\sigma_{\mathrm{z}}$ along the total embedded length of the fiber for the elastic pullout problem in the case of $\lambda=0.1$ and $\varepsilon=0.2$

\begin{tabular}{|c|c|c|}
\hline $\mathrm{z} / l$ & $\sigma_{\mathrm{Z}} / \sigma_{\mathrm{O}}$ (Numerical) & $\sigma_{\mathrm{z}} / \sigma_{\mathrm{o}}$ (Analytical) \\
\hline 0.00 & 1.016 & 1.000 \\
\hline 0.05 & 0.933 & 0.859 \\
\hline 0.10 & 0.758 & 0.738 \\
\hline 0.15 & 0.613 & 0.634 \\
\hline 0.20 & 0.516 & 0.544 \\
\hline 0.25 & 0.442 & 0.467 \\
\hline 0.30 & 0.384 & 0.400 \\
\hline 0.35 & 0.336 & 0.342 \\
\hline 0.40 & 0.295 & 0.292 \\
\hline 0.45 & 0.260 & 0.249 \\
\hline 0.50 & 0.230 & 0.211 \\
\hline 0.55 & 0.204 & 0.178 \\
\hline 0.60 & 0.182 & 0.149 \\
\hline 0.65 & 0.161 & 0.124 \\
\hline 0.70 & 0.143 & 0.101 \\
\hline 0.75 & 0.126 & 0.081 \\
\hline 0.80 & 0.110 & 0.063 \\
\hline 0.85 & 0.095 & 0.046 \\
\hline 0.90 & 0.076 & 0.030 \\
\hline 0.95 & 0.055 & 0.014 \\
\hline 1.00 & 0.040 & 0.000 \\
\hline
\end{tabular}


Table 3. Results of the distribution of normalized $\sigma_{z}$ along the total embedded length of the fiber for the elastic pullout problem in the case of $\lambda=0.01$ and $\varepsilon=0.1$.

\begin{tabular}{|c|c|c|}
\hline $\mathrm{z} / \mathrm{l}$ & $\sigma_{\mathrm{Z}} / \sigma_{\mathrm{o}}$ (Numerical) & $\sigma_{\mathrm{z}} / \sigma_{\mathrm{o}}$ (Analytical) \\
\hline 0.00 & 1.1580 & 1.0000 \\
\hline 0.05 & 0.3633 & 0.4713 \\
\hline 0.10 & 0.1697 & 0.2221 \\
\hline 0.15 & 0.0900 & 0.1046 \\
\hline 0.20 & 0.0526 & 0.0493 \\
\hline 0.25 & 0.0333 & 0.0232 \\
\hline 0.30 & 0.0225 & 0.0109 \\
\hline 0.35 & 0.0160 & 0.0051 \\
\hline 0.40 & 0.0119 & 0.0024 \\
\hline 0.45 & 0.0092 & 0.0011 \\
\hline 0.50 & 0.0073 & 0.0005 \\
\hline 0.55 & 0.0059 & 0.0002 \\
\hline 0.60 & 0.0049 & 0.0001 \\
\hline 0.65 & 0.0041 & 0.0000 \\
\hline 0.70 & 0.0032 & 0.0000 \\
\hline 0.75 & 0.0030 & 0.0000 \\
\hline 0.80 & 0.0026 & 0.0000 \\
\hline 0.85 & 0.0022 & 0.0000 \\
\hline 0.90 & 0.0019 & 0.0000 \\
\hline 0.95 & 0.0015 & 0.0000 \\
\hline 1.00 & 0.0003 & 0.0000 \\
\hline
\end{tabular}


Table 4. Results of the distribution of normalized $\sigma_{z}$ along the total embedded length of the fiber for the elastic pullout problem in the case of $\lambda=0.01$ and $\varepsilon$ $=0.01$.

\begin{tabular}{|c|c|c|}
\hline $\mathrm{z} / \mathrm{l}$ & $\sigma_{\mathrm{Z}} / \sigma_{\mathrm{o}}$ (Numerical) & $\sigma_{\mathrm{Z}} / \sigma_{\mathrm{o}}$ (Analytical) \\
\hline 0.00 & 1.051 & 1.000 \\
\hline 0.05 & 0.764 & 0.788 \\
\hline 0.10 & 0.598 & 0.621 \\
\hline 0.15 & 0.473 & 0.489 \\
\hline 0.20 & 0.378 & 0.386 \\
\hline 0.25 & 0.304 & 0.304 \\
\hline 0.30 & 0.247 & 0.239 \\
\hline 0.35 & 0.202 & 0.188 \\
\hline 0.40 & 0.166 & 0.148 \\
\hline 0.45 & 0.137 & 0.116 \\
\hline 0.50 & 0.114 & 0.091 \\
\hline 0.55 & 0.095 & 0.072 \\
\hline 0.60 & 0.079 & 0.056 \\
\hline 0.65 & 0.066 & 0.043 \\
\hline 0.70 & 0.055 & 0.033 \\
\hline 0.75 & 0.046 & 0.025 \\
\hline 0.80 & 0.038 & 0.018 \\
\hline 0.85 & 0.030 & 0.013 \\
\hline 0.90 & 0.022 & 0.008 \\
\hline 0.95 & 0.015 & 0.004 \\
\hline 1.00 & 0.000 & 0.000 \\
\hline
\end{tabular}


Table 5. Results of the distribution of normalized $\sigma_{\mathrm{z}}$ along the total embedded length of the fiber for the elastic pullout problem in the case of $\lambda=0.01$ and $\varepsilon$ $=0.001$.

\begin{tabular}{|c|c|c|}
\hline $\mathrm{z} / 1$ & $\sigma_{\mathrm{Z}} / \sigma_{\mathrm{o}}$ (Numerical) & $\sigma_{Z} / \sigma_{\mathrm{o}}$ (Analytical) \\
\hline 0.00 & 1.013 & 1.000 \\
\hline 0.05 & 0.928 & 0.919 \\
\hline 0.10 & 0.860 & 0.844 \\
\hline 0.15 & 0.796 & 0.774 \\
\hline 0.20 & 0.736 & 0.708 \\
\hline 0.25 & 0.679 & 0.646 \\
\hline 0.30 & 0.626 & 0.588 \\
\hline 0.35 & 0.575 & 0.533 \\
\hline 0.40 & 0.527 & 0.481 \\
\hline 0.45 & 0.481 & 0.432 \\
\hline 0.50 & 0.437 & 0.385 \\
\hline 0.55 & 0.394 & 0.341 \\
\hline 0.60 & 0.353 & 0.298 \\
\hline 0.65 & 0.313 & 0.257 \\
\hline 0.70 & 0.274 & 0.218 \\
\hline 0.75 & 0.235 & 0.179 \\
\hline 0.80 & 0.1970 & 0.142 \\
\hline 0.85 & 0.157 & 0.106 \\
\hline 0.90 & 0.117 & 0.070 \\
\hline 0.95 & 0.073 & 0.054 \\
\hline 1.00 & 0.002 & 0.000 \\
\hline
\end{tabular}


Table 6. Results of the distribution of normalized $\tau_{\mathrm{rz}}$ in the interface between the fiber and matrix along the total length of the fiber for the elastic pullout problem in the case of $\lambda=0.1$ and $\varepsilon=0.1$.

\begin{tabular}{|c|c|c|}
\hline $\mathrm{z} / \mathrm{l}$ & $\tau_{\mathrm{rz}} / \sigma_{\mathrm{o}}$ (Numerical) & $\tau_{\mathrm{rz}} / \sigma_{\mathrm{o}}$ (Analytical) \\
\hline 0.00 & -0.079 & -0.109 \\
\hline 0.05 & -0.125 & -0.098 \\
\hline 0.10 & -0.094 & -0.089 \\
\hline 0.15 & -0.074 & -0.080 \\
\hline 0.20 & -0.065 & -0.072 \\
\hline 0.25 & -0.056 & -0.066 \\
\hline 0.30 & -0.050 & -0.059 \\
\hline 0.35 & -0.044 & -0.054 \\
\hline 0.40 & -0.040 & -0.049 \\
\hline 0.45 & -0.036 & -0.045 \\
\hline 0.50 & -0.033 & -0.041 \\
\hline 0.55 & -0.030 & -0.038 \\
\hline 0.60 & -0.028 & -0.035 \\
\hline 0.65 & -0.026 & -0.033 \\
\hline 0.70 & -0.025 & -0.031 \\
\hline 0.75 & -0.024 & -0.029 \\
\hline 0.80 & -0.024 & -0.028 \\
\hline 0.85 & -0.024 & -0.027 \\
\hline 0.90 & -0.027 & -0.026 \\
\hline 0.95 & -0.032 & -0.025 \\
\hline 1.00 & -0.025 & -0.025 \\
\hline
\end{tabular}


Table 7. Results of the distribution of normalized $\tau_{\mathrm{rz}}$ in the interface between the fiber and matrix along the total length of the fiber for the elastic pullout problem in the case of $\lambda=0.1$ and $\varepsilon=0.2$.

\begin{tabular}{|c|c|c|}
\hline $\mathrm{z} / l$ & $\tau_{\mathrm{rz}} / \sigma_{\mathrm{o}}$ (Numerical) & $\tau_{\mathrm{rz}} / \sigma_{\mathrm{o}}$ (Analytical) \\
\hline 0.00 & -0.100 & -0.151 \\
\hline 0.05 & -0.176 & -0.130 \\
\hline 0.10 & -0.124 & -0.112 \\
\hline 0.15 & -0.093 & -0.096 \\
\hline 0.20 & -0.075 & -0.083 \\
\hline 0.25 & -0.061 & -0.071 \\
\hline 0.30 & -0.050 & -0.062 \\
\hline 0.35 & -0.042 & -0.053 \\
\hline 0.40 & -0.036 & -0.046 \\
\hline 0.45 & -0.031 & -0.040 \\
\hline 0.50 & -0.027 & -0.031 \\
\hline 0.55 & -0.023 & -0.030 \\
\hline 0.60 & -0.021 & -0.027 \\
\hline 0.65 & -0.018 & -0.023 \\
\hline 0.70 & -0.017 & -0.021 \\
\hline 0.75 & -0.015 & -0.019 \\
\hline 0.80 & -0.014 & -0.017 \\
\hline 0.85 & -0.014 & -0.016 \\
\hline 0.90 & -0.015 & -0.015 \\
\hline 0.95 & -0.018 & -0.015 \\
\hline 1.00 & -0.015 & -0.014 \\
\hline
\end{tabular}


Table 8. Results of the distribution of normalized $\tau_{\mathrm{rz}}$ in the interface between the fiber and matrix along the total length of the fiber for the elastic pullout problem in the case of $\lambda=0.01$ and $\varepsilon=0.1$.

\begin{tabular}{|c|c|c|}
\hline $\mathrm{z} / \mathrm{l}$ & $\tau_{\mathrm{rz}} / \sigma_{\mathrm{o}}$ (Numerical) & $\tau_{\mathrm{rz}} / \sigma_{\mathrm{o}}$ (Analytical) \\
\hline 0.00 & -0.1089 & -0.0752 \\
\hline 0.05 & -0.0293 & -0.0354 \\
\hline 0.10 & -0.0112 & -0.0167 \\
\hline 0.15 & -0.0050 & -0.0078 \\
\hline 0.20 & -0.0025 & -0.0037 \\
\hline 0.25 & -0.0013 & -0.0017 \\
\hline 0.30 & -0.0007 & -0.0008 \\
\hline 0.35 & -0.0004 & -0.0003 \\
\hline 0.40 & -0.0003 & -0.0001 \\
\hline 0.45 & -0.0002 & -0.0000 \\
\hline 0.50 & -0.0001 & -0.0000 \\
\hline 0.55 & -0.0001 & -0.0000 \\
\hline 0.60 & -0.0000 & -0.0000 \\
\hline 0.65 & -0.0000 & -0.0000 \\
\hline 0.70 & -0.0000 & -0.0000 \\
\hline 0.75 & -0.0000 & -0.0000 \\
\hline 0.80 & -0.0000 & -0.0000 \\
\hline 0.85 & -0.0000 & -0.0000 \\
\hline 0.90 & -0.0000 & -0.0000 \\
\hline 0.95 & -0.0000 & -0.0000 \\
\hline 1.00 & -0.0000 & -0.0000 \\
\hline
\end{tabular}


Table 9. Results of the distribution of normalized $\tau_{\mathrm{rz}}$ in the interface between the fiber and matrix along the total length of the fiber for the elastic pullout problem in the case of $\lambda=0.01$ and $\varepsilon=0.01$.

\begin{tabular}{|c|c|c|}
\hline $\mathrm{z} / l$ & $\tau_{\mathrm{rz}} / \sigma_{\mathrm{o}}$ (Numerical) & $\tau_{\mathrm{rz}} / \sigma_{\mathrm{o}}$ (Analytical) \\
\hline 0.00 & -0.0327 & -0.0237 \\
\hline 0.05 & -0.0184 & -0.0187 \\
\hline 0.10 & -0.0137 & -0.0147 \\
\hline 0.15 & -0.0104 & -0.0116 \\
\hline 0.20 & -0.0079 & -0.0091 \\
\hline 0.25 & -0.0062 & -0.0072 \\
\hline 0.30 & -0.0048 & -0.0057 \\
\hline 0.35 & -0.0038 & -0.0045 \\
\hline 0.40 & -0.0030 & -0.0035 \\
\hline 0.45 & -0.0024 & -0.0028 \\
\hline 0.50 & -0.0020 & -0.0022 \\
\hline 0.55 & -0.0016 & -0.0017 \\
\hline 0.60 & -0.0013 & -0.0014 \\
\hline 0.65 & -0.0011 & -0.0011 \\
\hline 0.70 & -0.0009 & -0.0009 \\
\hline 0.75 & -0.0008 & -0.0007 \\
\hline 0.80 & -0.0007 & -0.0006 \\
\hline 0.85 & -0.0007 & -0.0005 \\
\hline 0.90 & -0.0007 & -0.0004 \\
\hline 0.95 & -0.0008 & -0.0004 \\
\hline 1.00 & -0.0015 & -0.0004 \\
\hline
\end{tabular}


Table 10. Results of the distribution of normalized $\tau_{\mathrm{rz}}$ in the interface between the fiber and matrix along the total length of the fiber for the elastic pullout problem in the case of $\lambda=0.01$ and $\varepsilon=0.001$.

\begin{tabular}{|c|c|c|}
\hline $\mathrm{z} / \mathrm{l}$ & $\tau_{\mathrm{rz}} / \sigma_{\mathrm{o}}$ (Numerical) & $\tau_{\mathrm{rz}} / \sigma_{\mathrm{o}}$ (Analytical) \\
\hline 0.00 & -0.0085 & -0.0083 \\
\hline 0.05 & -0.0066 & -0.0077 \\
\hline 0.10 & -0.0063 & -0.0072 \\
\hline 0.15 & -0.0059 & -0.0068 \\
\hline 0.20 & -0.0056 & -0.0063 \\
\hline 0.25 & -0.0052 & -0.0060 \\
\hline 0.30 & -0.0050 & -0.0056 \\
\hline 0.35 & -0.0047 & -0.0053 \\
\hline 0.40 & -0.0045 & -0.0050 \\
\hline 0.45 & -0.0043 & -0.0047 \\
\hline 0.50 & -0.0041 & -0.0045 \\
\hline 0.55 & -0.0040 & -0.0043 \\
\hline 0.60 & -0.0038 & -0.0041 \\
\hline 0.65 & -0.0037 & -0.0040 \\
\hline 0.70 & -0.0037 & -0.0038 \\
\hline 0.75 & -0.0037 & -0.0037 \\
\hline 0.80 & -0.0037 & -0.0036 \\
\hline 0.85 & -0.0038 & -0.0036 \\
\hline 0.90 & -0.0040 & -0.0035 \\
\hline 0.95 & -0.0044 & -0.0035 \\
\hline 1.00 & -0.0073 & -0.0035 \\
\hline
\end{tabular}


Table 11. Value of the normalized maximum displacement for all the relations $\lambda$ and $\varepsilon$ considered in the numerical analysis of the elastic pullout problem.

\begin{tabular}{lll}
\hline$\lambda$ & $\varepsilon$ & $\frac{W_{\max } E_{f}}{\sigma_{o} l}$ \\
0.1 & 0.1 & 0.5704 \\
0.1 & 0.2 & 0.3835 \\
0.01 & 0.1 & 0.0616 \\
0.01 & 0.01 & 0.2321 \\
0.01 & 0.001 & 0.8996 \\
\hline
\end{tabular}




\section{RESULTS OF THE DEBONDING PROBLEM}

All the results of the debonding problem are presented here. The results of the relation between the load applied and the length of debonding are given in Tables 12 to 16 . The distribution of the normal stresses are presented in Tables 17 to 21 and the distribution of the shear stresses are compiled in Tables 22 to 26 . 
Table 12. Results of the relation between the normalized load applied and the normalized length of debonding for the case of $\lambda=0.1$ and $\varepsilon=0.1$.

\begin{tabular}{|c|c|c|}
\hline$l_{D} / l$ & $\sigma_{\mathrm{o}} / \tau_{\mathrm{s}}$ (Numerical) & $\sigma_{\mathrm{o}} / \tau_{\mathrm{s}}$ (Analytical) \\
\hline 0.00 & 6.660 & 9.137 \\
\hline 0.05 & 9.280 & 8.988 \\
\hline 0.10 & 9.135 & 8.825 \\
\hline 0.15 & 8.590 & 8.646 \\
\hline 0.20 & 8.129 & 8.449 \\
\hline 0.25 & 7.843 & 8.231 \\
\hline 0.30 & 7.655 & 7.990 \\
\hline 0.35 & 7.500 & 7.721 \\
\hline 0.40 & 7.356 & 7.422 \\
\hline 0.45 & 7.210 & 7.088 \\
\hline 0.50 & 7.065 & 6.715 \\
\hline 0.55 & 6.900 & 6.297 \\
\hline 0.60 & 6.700 & 5.830 \\
\hline 0.65 & 6.466 & 5.308 \\
\hline 0.70 & 6.173 & 4.725 \\
\hline 0.75 & 5.779 & 4.076 \\
\hline 0.80 & 5.268 & 3.353 \\
\hline 0.85 & 4.412 & 2.530 \\
\hline 0.90 & 3.370 & \\
\hline 0.95 & 3.104 & \\
\hline 1.00 & 2.660 & \\
\hline
\end{tabular}


Table 13. Results of the relation between the normalized load applied and the normalized length of debonding for the case of $\lambda=0.1$ and $\varepsilon=0.2$.

\begin{tabular}{|c|c|c|}
\hline$l_{D} / l$ & $\sigma_{\mathrm{o}} / \tau_{\mathrm{s}}$ (Numerical) & $\sigma_{\mathrm{o}} / \tau_{\mathrm{s}}$ (Analytical) \\
\hline 0.00 & 4.495 & 6.614 \\
\hline 0.05 & 6.250 & 6.532 \\
\hline 0.10 & 6.350 & 6.442 \\
\hline 0.15 & 6.310 & 6.344 \\
\hline 0.20 & 6.121 & 6.237 \\
\hline 0.25 & 5.962 & 6.118 \\
\hline 0.30 & 5.946 & 5.986 \\
\hline 0.35 & 5.801 & 5.838 \\
\hline 0.40 & 5.741 & 5.671 \\
\hline 0.45 & 5.688 & 5.480 \\
\hline 0.50 & 5.634 & 5.261 \\
\hline 0.55 & 5.574 & 5.008 \\
\hline 0.60 & 5.550 & 4.713 \\
\hline 0.65 & 5.405 & 4.369 \\
\hline 0.70 & 5.272 & 3.964 \\
\hline 0.75 & 5.073 & 3.485 \\
\hline 0.80 & 4.769 & 2.915 \\
\hline 0.85 & 4.236 & 2.207 \\
\hline 0.90 & 3.446 & \\
\hline 0.95 & 3.173 & \\
\hline 1.00 & 2.688 & \\
\hline
\end{tabular}


Table 14. Results of the relation between the normalized load applied and the normalized length of debonding for the case of $\lambda=0.01$ and $\varepsilon=0.1$.

\begin{tabular}{|c|c|c|}
\hline$l_{D} / l$ & $\sigma_{\mathrm{o}} / \tau_{\mathrm{s}}$ (Numerical) & $\sigma_{\mathrm{o}} / \tau_{\mathrm{s}}$ (Analytical) \\
\hline 0.00 & 13.252 & 13.293 \\
\hline 0.05 & 13.205 & 13.219 \\
\hline 0.10 & 13.140 & 13.141 \\
\hline 0.15 & 13.100 & 13.057 \\
\hline 0.20 & 13.090 & 12.967 \\
\hline 0.25 & 13.085 & 12.872 \\
\hline 0.30 & 13.083 & 12.768 \\
\hline 0.35 & 13.081 & 12.656 \\
\hline 0.40 & 13.079 & 12.535 \\
\hline 0.45 & 13.076 & 12.401 \\
\hline 0.50 & 13.074 & 12.252 \\
\hline 0.55 & 13.074 & 12.086 \\
\hline 0.60 & 13.074 & 11.898 \\
\hline 0.65 & 13.074 & 11.680 \\
\hline 0.70 & 13.074 & 11.424 \\
\hline 0.75 & 13.074 & 11.111 \\
\hline 0.80 & 13.050 & 10.709 \\
\hline 0.85 & 13.025 & 10.137 \\
\hline 0.90 & 13.000 & 9.137 \\
\hline 0.95 & 12.413 & 6.715 \\
\hline 1.00 & 7.390 & \\
\hline
\end{tabular}


Table 15. Results of the relation between the normalized load applied and the normalized length of debonding for the case of $\lambda=0.01$ and $\varepsilon=0.01$.

\begin{tabular}{|c|c|c|}
\hline$l_{D} / l$ & $\sigma_{\mathrm{o}} / \tau_{\mathrm{s}}$ (Numerical) & $\sigma_{\mathrm{o}} / \tau_{\mathrm{s}}$ (Analytical) \\
\hline 0.00 & 33.310 & 42.033 \\
\hline 0.05 & 42.870 & 41.795 \\
\hline 0.10 & 41.350 & 41.541 \\
\hline 0.15 & 41.000 & 41.268 \\
\hline 0.20 & 40.830 & 40.974 \\
\hline 0.25 & 40.830 & 40.653 \\
\hline 0.30 & 40.830 & 40.299 \\
\hline 0.35 & 40.830 & 39.904 \\
\hline 0.40 & 40.830 & 39.453 \\
\hline 0.45 & 40.830 & 38.929 \\
\hline 0.50 & 40.830 & 38.304 \\
\hline 0.55 & 40.750 & 37.538 \\
\hline 0.60 & 40.500 & 36.569 \\
\hline 0.65 & 40.250 & 35.305 \\
\hline 0.70 & 40.000 & 33.610 \\
\hline 0.75 & 39.220 & 31.285 \\
\hline 0.80 & 37.000 & 28.052 \\
\hline 0.85 & 32.000 & 23.561 \\
\hline 0.90 & 25.000 & 17.444 \\
\hline 0.95 & 15.000 & 9.493 \\
\hline 1.00 & 4.080 & \\
\hline
\end{tabular}


Table 16. Results of the relation between the normalized load applied and the nor, alized length of debonding for the case of $\lambda=0.01$ and $\varepsilon=0.001$.

\begin{tabular}{|c|c|c|}
\hline$l_{D} / 1$ & $\sigma_{\mathrm{o}} / \tau_{\mathrm{s}}$ (Numerical) & $\sigma_{\mathrm{o}} / \tau_{\mathrm{s}}$ (Analytical) \\
\hline 0.00 & 126.900 & 120.436 \\
\hline 0.05 & 117.700 & 118.071 \\
\hline 0.10 & 108.270 & 115.461 \\
\hline 0.15 & 104.200 & 112.583 \\
\hline 0.20 & 100.100 & 109.407 \\
\hline 0.25 & 95.740 & 105.906 \\
\hline 0.30 & 92.500 & 102.049 \\
\hline 0.35 & 89.000 & 97.805 \\
\hline 0.40 & 86.100 & 93.144 \\
\hline 0.45 & 83.200 & 88.037 \\
\hline 0.50 & 78.950 & 82.456 \\
\hline 0.55 & 73.000 & 76.379 \\
\hline 0.60 & 68.500 & 69.787 \\
\hline 0.65 & 63.800 & 62.671 \\
\hline 0.70 & 58.500 & 55.031 \\
\hline 0.75 & 53.430 & 46.879 \\
\hline 0.80 & 44.000 & 38.242 \\
\hline 0.85 & 33.500 & 29.162 \\
\hline 0.90 & 23.500 & 19.703 \\
\hline 0.95 & 12.500 & 9.943 \\
\hline 1.00 & 2.940 & \\
\hline
\end{tabular}


Table 17. Results of the distribution of normalized $\sigma_{\mathrm{z}}$ along the total embedded length of the fiber for the debonding problem in the case of $\lambda=0.1, \varepsilon=0.1$ and $l_{D}=1$.

\begin{tabular}{|c|c|c|}
\hline $\mathrm{z} / l$ & $\sigma_{\mathrm{z}} / \tau_{\mathrm{s}}$ (Numerical) & $\sigma_{\mathrm{z}} / \tau_{\mathrm{s}}$ (Analytical) \\
\hline 0.00 & 9.154 & 8.825 \\
\hline 0.05 & 9.059 & 8.825 \\
\hline 0.10 & 8.375 & 8.825 \\
\hline 0.15 & 7.697 & 7.875 \\
\hline 0.20 & 6.959 & 7.019 \\
\hline 0.25 & 6.616 & 6.247 \\
\hline 0.30 & 5.468 & 5.548 \\
\hline 0.35 & 4.893 & 4.915 \\
\hline 0.40 & 4.397 & 4.341 \\
\hline 0.45 & 3.961 & 3.818 \\
\hline 0.50 & 3.573 & 3.340 \\
\hline 0.55 & 3.223 & 2.903 \\
\hline 0.60 & 2.903 & 2.499 \\
\hline 0.65 & 2.608 & 2.126 \\
\hline 0.70 & 2.332 & 1.777 \\
\hline 0.75 & 2.070 & 1.450 \\
\hline 0.80 & 1.813 & 1.139 \\
\hline 0.85 & 1.548 & 0.843 \\
\hline 0.90 & 1.213 & 0.556 \\
\hline 0.95 & 0.797 & 0.276 \\
\hline 1.00 & 0.542 & 0.000 \\
\hline
\end{tabular}


Table 18. Results of the distribution of normalized $\sigma_{\mathrm{z}}$ along the total embedded length of the fiber for the debonding problem in the case of $\lambda=0.1, \varepsilon=0.2$ and $l_{D}=1$.

\begin{tabular}{|c|c|c|}
\hline $\mathrm{z} / l$ & $\sigma_{\mathrm{z}} / \tau_{\mathrm{s}}$ (Numerical) & $\sigma_{\mathrm{z}} / \tau_{\mathrm{s}}$ (Analytical) \\
\hline 0.00 & 6.343 & 6.442 \\
\hline 0.05 & 6.238 & 6.442 \\
\hline 0.10 & 5.572 & 6.442 \\
\hline 0.15 & 4.928 & 5.515 \\
\hline 0.20 & 4.249 & 4.718 \\
\hline 0.25 & 3.569 & 4.034 \\
\hline 0.30 & 3.014 & 3.446 \\
\hline 0.35 & 2.584 & 2.939 \\
\hline 0.40 & 2.237 & 2.503 \\
\hline 0.45 & 1.951 & 2.126 \\
\hline 0.50 & 1.710 & 1.799 \\
\hline 0.55 & 1.505 & 1.516 \\
\hline 0.60 & 1.327 & 1.268 \\
\hline 0.65 & 1.171 & 1.050 \\
\hline 0.70 & 1.033 & 0.858 \\
\hline 0.75 & 0.908 & 0.686 \\
\hline 0.80 & 0.791 & 0.530 \\
\hline 0.85 & 0.676 & 0.387 \\
\hline 0.90 & 0.544 & 0.253 \\
\hline 0.95 & 0.389 & 0.125 \\
\hline 1.00 & 0.282 & 0.000 \\
\hline
\end{tabular}


Table 19. Results of the distribution of normalized $\sigma_{\mathrm{z}}$ along the total embedded length of the fiber for the debonding problem in the case of $\lambda=0.01, \varepsilon=0.1$ and $l_{D}=1$.

\begin{tabular}{|c|c|c|}
\hline $\mathrm{z} / \mathrm{l}$ & $\sigma_{\mathrm{z}} / \tau_{\mathrm{s}}$ (Numerical) & $\sigma_{z} / \tau_{\mathrm{s}}$ (Analytical) \\
\hline 0.00 & 13.141 & 13.141 \\
\hline 0.05 & 13.131 & 13.141 \\
\hline 0.10 & 11.255 & 13.141 \\
\hline 0.15 & 3.989 & 6.139 \\
\hline 0.20 & 1.738 & 2.868 \\
\hline 0.25 & 0.892 & 1.340 \\
\hline 0.30 & 0.514 & 0.626 \\
\hline 0.35 & 0.352 & 0.292 \\
\hline 0.40 & 0.221 & 0.136 \\
\hline 0.45 & 0.160 & 0.063 \\
\hline 0.50 & 0.120 & 0.029 \\
\hline 0.55 & 0.094 & 0.013 \\
\hline 0.60 & 0.076 & 0.006 \\
\hline 0.65 & 0.062 & 0.003 \\
\hline 0.70 & 0.052 & 0.001 \\
\hline 0.75 & 0.044 & 0.000 \\
\hline 0.80 & 0.038 & 0.000 \\
\hline 0.85 & 0.033 & 0.000 \\
\hline 0.90 & 0.028 & 0.000 \\
\hline 0.95 & 0.022 & 0.000 \\
\hline 1.00 & 0.004 & 0.000 \\
\hline
\end{tabular}


Table 20. Results of the distribution of normalized $\sigma_{\mathrm{z}}$ along the total embedded length of the fiber for the debonding problem in the case of $\lambda=0.01, \varepsilon=0.01$ and $l_{D}=1$.

\begin{tabular}{|c|c|c|}
\hline $\mathrm{z} / \mathrm{l}$ & $\sigma_{\mathrm{z}} / \tau_{\mathrm{s}}$ (Numerical) & $\sigma_{\mathrm{z}} / \tau_{\mathrm{s}}$ (Analytical) \\
\hline 0.00 & 41.357 & 41.541 \\
\hline 0.05 & 41.296 & 41.541 \\
\hline 0.10 & 39.584 & 41.541 \\
\hline 0.15 & 29.202 & 32.653 \\
\hline 0.20 & 22.175 & 25.664 \\
\hline 0.25 & 17.281 & 20.170 \\
\hline 0.30 & 13.676 & 15.848 \\
\hline 0.35 & 10.947 & 12.450 \\
\hline 0.40 & 8.843 & 9.775 \\
\hline 0.45 & 7.198 & 7.670 \\
\hline 0.50 & 5.898 & 6.010 \\
\hline 0.55 & 4.858 & 4.701 \\
\hline 0.60 & 4.017 & 3.665 \\
\hline 0.65 & 3.331 & 2.842 \\
\hline 0.70 & 2.757 & 2.185 \\
\hline 0.75 & 2.272 & 1.655 \\
\hline 0.80 & 1.848 & 1.221 \\
\hline 0.85 & 1.464 & 0.859 \\
\hline 0.90 & 1.095 & 0.546 \\
\hline 0.95 & 0.707 & 0.265 \\
\hline 1.00 & 0.003 & 0.000 \\
\hline
\end{tabular}


Table 21. Results of the distribution of normalized $\sigma_{\mathrm{z}}$ along the total embedded length of the fiber for the debonding problem in the case of $\lambda=0.01, \varepsilon=0.001$ and $l_{D}=1$.

\begin{tabular}{|c|c|c|}
\hline $\mathrm{z} / \mathrm{l}$ & $\sigma_{\mathrm{z}} / \tau_{\mathrm{s}}$ (Numerical) & $\sigma_{\mathrm{z}} / \tau_{\mathrm{s}}$ (Analytical) \\
\hline 0.00 & 108.278 & 115.461 \\
\hline 0.05 & 108.333 & 115.461 \\
\hline 0.10 & 106.885 & 115.461 \\
\hline 0.15 & 97.135 & 105.786 \\
\hline 0.20 & 88.609 & 96.724 \\
\hline 0.25 & 81.145 & 88.222 \\
\hline 0.30 & 74.346 & 80.231 \\
\hline 0.35 & 68.047 & 72.705 \\
\hline 0.40 & 62.147 & 65.601 \\
\hline 0.45 & 56.578 & 58.876 \\
\hline 0.50 & 51.285 & 52.492 \\
\hline 0.55 & 46.222 & 46.413 \\
\hline 0.60 & 41.348 & 40.602 \\
\hline 0.65 & 36.624 & 35.027 \\
\hline 0.70 & 32.008 & 29.655 \\
\hline 0.75 & 27.459 & 24.454 \\
\hline 0.80 & 22.926 & 19.395 \\
\hline 0.85 & 18.347 & 14.449 \\
\hline 0.90 & 13.624 & 9.586 \\
\hline 0.95 & 8.560 & 4.779 \\
\hline 1.00 & -1.608 & 0.000 \\
\hline
\end{tabular}


Table 22. results of the distribution of normalized $\tau_{\mathrm{rz}}$ in the interface between the fiber and matrix along the total length of the fiber for the debonding problem in the case of $\lambda=0.1, \varepsilon=0.1$ and $l_{D}=1$.

\begin{tabular}{|c|c|c|}
\hline $\mathrm{z} / \mathrm{l}$ & $\tau_{\mathrm{rz}} / \tau_{\mathrm{s}}$ (Numerical) & $\tau_{\mathrm{rz}} / \tau_{\mathrm{s}}$ (Analytical) \\
\hline 0.00 & 0.002 & 0.000 \\
\hline 0.05 & 0.176 & 0.000 \\
\hline 0.10 & -0.972 & -1.000 \\
\hline 0.15 & -1.000 & -0.901 \\
\hline 0.20 & -0.852 & -0.812 \\
\hline 0.25 & -0.698 & -0.734 \\
\hline 0.30 & -0.594 & -0.664 \\
\hline 0.35 & -0.513 & -0.602 \\
\hline 0.40 & -0.450 & -0.547 \\
\hline 0.45 & -0.400 & -0.499 \\
\hline 0.50 & -0.360 & -0.456 \\
\hline 0.55 & -0.327 & -0.419 \\
\hline 0.60 & -0.300 & -0.387 \\
\hline 0.65 & -0.278 & -0.360 \\
\hline 0.70 & -0.262 & -0.337 \\
\hline 0.75 & -0.251 & -0.318 \\
\hline 0.80 & -0.246 & -0.302 \\
\hline 0.85 & -0.248 & -0.291 \\
\hline 0.90 & -0.277 & -0.282 \\
\hline 0.95 & -0.328 & -0.277 \\
\hline 1.00 & -0.259 & -0.276 \\
\hline
\end{tabular}


Table 23. Results of the distribution of normalized $\tau_{\mathrm{rz}}$ in the interface between the fiber and matrix along the total length of the fiber for the debonding problem in the case of $\lambda=0.1, \varepsilon=0.2$ and $l_{D}=1$.

\begin{tabular}{|c|c|c|}
\hline $\mathrm{z} / 1$ & $\tau_{\mathrm{rz}} / \tau_{\mathrm{s}}$ (Numerical) & $\tau_{\mathrm{rz}} / \tau_{\mathrm{s}}$ (Analytical) \\
\hline 0.00 & -0.005 & 0.000 \\
\hline 0.05 & 0.179 & 0.000 \\
\hline 0.10 & -1.000 & -1.000 \\
\hline 0.15 & -0.941 & -0.858 \\
\hline 0.20 & -0.741 & -0.737 \\
\hline 0.25 & -0.566 & -0.633 \\
\hline 0.30 & -0.451 & -0.545 \\
\hline 0.35 & -0.365 & -0.469 \\
\hline 0.40 & -0.302 & -0.405 \\
\hline 0.45 & -0.253 & -0.350 \\
\hline 0.50 & -0.215 & -0.303 \\
\hline 0.55 & -0.185 & -0.264 \\
\hline 0.60 & -0.161 & -0.231 \\
\hline 0.65 & -0.142 & -0.204 \\
\hline 0.70 & -0.128 & -0.181 \\
\hline 0.75 & -0.117 & -0.163 \\
\hline 0.80 & -0.109 & -0.148 \\
\hline 0.85 & -0.107 & -0.138 \\
\hline 0.90 & -0.113 & -0.130 \\
\hline 0.95 & -0.129 & -0.126 \\
\hline 1.00 & -0.106 & -0.124 \\
\hline
\end{tabular}


Table 24. Results of the distribution of normalized $\tau_{\mathrm{rz}}$ in the interface between the fiber and matrix along the total length of the fiber for the debonding problem in the case of $\lambda=0.01, \varepsilon=0.1$ and $l_{D}=1$.

\begin{tabular}{|c|c|c|}
\hline $\mathrm{z} / \mathrm{l}$ & $\tau_{\mathrm{rz}} / \tau_{\mathrm{s}}$ (Numerical) & $\tau_{\mathrm{rz}} / \tau_{\mathrm{s}}$ (Analytical) \\
\hline 0.00 & -0.0010 & 0.0000 \\
\hline 0.05 & 0.0010 & 0.0000 \\
\hline 0.10 & -1.2236 & -1.0000 \\
\hline 0.15 & -0.3614 & -0.4672 \\
\hline 0.20 & -0.1231 & -0.2182 \\
\hline 0.25 & -0.0518 & -0.1019 \\
\hline 0.30 & -0.0247 & -0.0476 \\
\hline 0.35 & -0.0130 & -0.0222 \\
\hline 0.40 & -0.0075 & -0.0104 \\
\hline 0.45 & -0.0046 & -0.0048 \\
\hline 0.50 & -0.0030 & -0.0022 \\
\hline 0.55 & -0.0020 & -0.0010 \\
\hline 0.60 & -0.0014 & -0.0004 \\
\hline 0.65 & -0.0011 & -0.0002 \\
\hline 0.70 & -0.0008 & -0.0001 \\
\hline 0.75 & -0.0006 & -0.0000 \\
\hline 0.80 & -0.0005 & -0.0000 \\
\hline 0.85 & -0.0004 & -0.0000 \\
\hline 0.90 & -0.0004 & -0.0000 \\
\hline 0.95 & -0.0007 & -0.0000 \\
\hline 1.00 & -0.0023 & -0.0000 \\
\hline
\end{tabular}


Table 25. Results of the distribution of normalized $\tau_{\mathrm{rz}}$ in the interface between the fiber and matrix along the total length of the fiber for the debonding problem in the case of $\lambda=0.01, \varepsilon=0.01$ and $l_{D}=1$.

\begin{tabular}{|c|c|c|}
\hline $\mathrm{z} / \mathrm{l}$ & $\tau_{\mathrm{rz}} / \tau_{\mathrm{s}}$ (Numerical) & $\tau_{\mathrm{rz}} / \tau_{\mathrm{s}}$ (Analytical) \\
\hline 0.00 & -0.005 & 0.000 \\
\hline 0.05 & -0.002 & 0.000 \\
\hline 0.10 & -1.000 & -1.000 \\
\hline 0.15 & -0.830 & -0.786 \\
\hline 0.20 & -0.552 & -0.618 \\
\hline 0.25 & -0.399 & -0.486 \\
\hline 0.30 & -0.299 & -0.382 \\
\hline 0.35 & -0.229 & -0.300 \\
\hline 0.40 & -0.177 & -0.236 \\
\hline 0.45 & -0.139 & -0.186 \\
\hline 0.50 & -0.111 & -0.147 \\
\hline 0.55 & -0.089 & -0.116 \\
\hline 0.60 & -0.072 & -0.092 \\
\hline 0.65 & -0.060 & -0.073 \\
\hline 0.70 & -0.050 & -0.058 \\
\hline 0.75 & -0.043 & -0.047 \\
\hline 0.80 & -0.038 & -0.039 \\
\hline 0.85 & -0.035 & -0.034 \\
\hline 0.90 & -0.035 & -0.029 \\
\hline 0.95 & -0.039 & -0.027 \\
\hline 1.00 & -0.028 & -0.026 \\
\hline
\end{tabular}


Table 26. Results of the distribution of normalized $\tau_{\mathrm{rz}}$ in the interface between the fiber and matrix along the total length of the fiber for the debonding problem in the case of $\lambda=0.01, \varepsilon=0.001$ and $l_{D}=1$.

\begin{tabular}{|c|c|c|}
\hline $\mathrm{z} / \mathrm{l}$ & $\tau_{\mathrm{rz}} / \tau_{\mathrm{s}}$ (Numerical) & $\tau_{\mathrm{rz}} / \tau_{\mathrm{s}}$ (Analytical) \\
\hline 0.00 & -0.003 & 0.000 \\
\hline 0.05 & -0.001 & 0.000 \\
\hline 0.10 & -0.754 & -1.000 \\
\hline 0.15 & -0.898 & -0.935 \\
\hline 0.20 & -0.758 & -0.877 \\
\hline 0.25 & -0.681 & -0.823 \\
\hline 0.30 & -0.627 & -0.775 \\
\hline 0.35 & -0.584 & -0.730 \\
\hline 0.40 & -0.550 & -0.690 \\
\hline 0.45 & -0.521 & -0.654 \\
\hline 0.50 & -0.496 & -0.622 \\
\hline 0.55 & -0.476 & -0.593 \\
\hline 0.60 & -0.460 & -0.568 \\
\hline 0.65 & -0.448 & -0.546 \\
\hline 0.70 & -0.439 & -0.530 \\
\hline 0.75 & -0.435 & -0.512 \\
\hline 0.80 & -0.436 & -0.499 \\
\hline 0.85 & -0.444 & -0.489 \\
\hline 0.90 & -0.465 & -0.483 \\
\hline 0.95 & -0.514 & -0.478 \\
\hline 1.00 & -0.028 & -0.477 \\
\hline
\end{tabular}




\section{COMPARISON OF MAXIMUM DISPLACEMENT}

The results of the comparison of the bed coefficient in the elastic pullout problem are given in Table 27.

Table 27. Comparison of maximum displacement using the dimensionless expression of the bed coefficient for all the relations $\lambda$ and $\varepsilon$ considered in the elastic pullout problem.

\begin{tabular}{llll}
\hline$\lambda$ & $\varepsilon$ & $\bar{B}_{\text {analytic }}$ & $\bar{B}_{\text {numeric }}$ \\
\hline 0.1 & 0.1 & 0.6497 & 0.5506 \\
0.1 & 0.2 & 0.9406 & 0.8190 \\
0.01 & 0.1 & 0.4726 & 0.5096 \\
0.01 & 0.01 & 0.1494 & 0.1353 \\
0.01 & 0.001 & 0.0428 & 0.0349 \\
& & & \\
\hline
\end{tabular}

